\title{
Resolvent conditions for the control of unitary groups and their approximations
}

\author{
Luc Miller ${ }^{1}$
}

\begin{abstract}
A self-adjoint operator $\mathcal{A}$ and an operator $\mathcal{C}$ bounded from the domain $\mathscr{D}(\mathcal{A})$ with the graph norm to another Hilbert space are considered. The admissibility or the exact observability in finite time of the unitary group generated by $i \mathcal{A}$ with respect to the observation operator $\mathcal{C}$ are characterized by some spectral inequalities on $\mathcal{A}$ and $\mathcal{C}$. E.g. both properties hold if and only if $x \mapsto\|(\mathcal{A}-\lambda) x\|+\|\mathcal{C} x\|$ is a norm on $\mathscr{D}(\mathcal{A})$ equivalent to $x \mapsto\|(\mathcal{A}-\lambda) x\|+\|x\|$ uniformly with respect to $\lambda \in \mathbb{R}$.

This paper generalizes and simplifies some results on the control of unitary groups obtained using these so-called resolvent conditions, also known as Hautus tests. It proves new theorems on the equivalence (with respect to admissibility and observability) between first and second order equations, between groups generated by $i \mathcal{A}$ and $i f(\mathcal{A})$ for positive $\mathcal{A}$ and convex $f$, and between a group and its Galerkin approximations. E.g. they apply to the control of linear Schrödinger, wave and plates equations and to the uniform control of their finite element semi-discretization.
\end{abstract}

Mathematics Subject Classification (2010). 93B05, 93B07, 93C20, 65J10, 47D06.

Keywords. Observability of PDEs, infinite dimensional Hautus test, Galerkin approximation of control problems.

\section{Contents}

1 Introduction ......................... 2

2 Resolvent conditions for admissibility and observability . . . . . . . . 6

3 Links between systems with generator build on a positive $A$. . . . . . . . 21

4 Semidiscretization of a system with positive $A \ldots$. . . . . . . . . 32

References ..................... 52

\footnotetext{
${ }^{1}$ The author wishes to thank Institut Henri Poincaré (Paris, France) for providing a very stimulating environment during the "Control of Partial and Differential Equations and Applications" program in the Fall 2010. This work was partially supported by the "Agence Nationale de la Recherche" (ANR), Project CISIFS, grant ANR-09-BLAN-0213-02. The encouragement of Thomas Duyckaerts was instrumental in finalizing this text.
} 


\section{Introduction}

1.1. Resolvent conditions. The notion of resolvent condition considered in this paper was introduced in control theory by David Russell and George Weiss in [40] as an infinite-dimensional version, for exponentially stable semigroups, of the Hautus test for controllability. Although this paper is self-contained, we refer to the monograph [42] for an introduction to and a full account of the control theory of semigroups, e.g. admissibility, exact controllability and the Hautus test, with applications to PDEs. This paper deals with the controllability of unitary groups rather than exponentially stable semigroups. We refer to [22] for the history of this latter issue since [40] and the extension of the results on unitary groups in [30] to more general groups. We refer to [8] for the investigation of resolvent conditions for parabolic semigroups, using $\$ 3.2$ of the present paper.

Readers more familiar with the spectral theory of semigroups may consider these resolvent conditions as analogous to the better known resolvent condition for exponential stability [9], Theorem V.1.11, due to Jan Prüss [34], Fa Lun Huang [20] and Günther Greiner (after a key result on contraction semigroup by Larry Gearhart [15] generalized to any semigroup in [17] and [19]). Indeed the growth abscissa of a semigroup $\left(e^{t \mathscr{E}}\right)_{t \geqslant 0}$ satisfies [34], Proposition 2:

$$
\omega_{0}(\mathscr{G}):=\inf _{t>0} \ln \frac{\left\|e^{t \mathscr{G}}\right\|}{t}=\inf \left\{\omega \in \mathbb{R} \mid \sup _{\Re \lambda \geqslant \omega}\left\|(\lambda-\mathscr{G})^{-1}\right\|<\infty\right\} .
$$

The Greiner-Huang-Prüss test [34], Corollary 4, for exponential stability follows:

$$
\omega_{0}(\mathscr{E})<0 \Longleftrightarrow \exists M>0, \forall \lambda \in \mathbb{C} \text { such that } \Re \lambda>0,\left\|(\lambda-\mathscr{G})^{-1}\right\| \leqslant M \text {. }
$$

The analogous result for exact controllability of unitary group, or equivalently exact observability, is stated precisely in Theorem 2.4. A self-adjoint operator $\mathcal{A}$ on a Hilbert space $X$ and an operator $\ell$ bounded from the domain $D(\mathcal{A})$ with the graph norm to another Hilbert space are considered. The resolvent condition involved for the exact observability in finite time of the unitary group generated by $i \mathcal{A}$ with respect to the observation operator $\mathcal{C}$ is

$$
\exists M>0, m>0, \forall x \in \mathscr{D}(\mathcal{A}), \lambda \in \mathbb{R}, \quad\|x\|^{2} \leqslant M\|(\mathcal{A}-\lambda) x\|^{2}+m\|\mathcal{C} x\|^{2} .
$$

1.2. Outline. Starting from the basic resolvent conditions characterizing admissibility in Theorem 2.3 and exact controllability in Theorem 2.4, this paper investigates various other forms of resolvent conditions, with variable coefficients, with restricted spectral parameter, with fractional powers of the generator. A quasimode approach to disproving exact controllability is introduced in $\$ 2.7$. In $\$ 3$ and $\S 4$, resolvent conditions are applied to unitary groups $\left(e^{i t \mathcal{A}}\right)_{t \in \mathbb{R}}$ with various positive $\mathcal{A}$ build on the same positive self-adjoint operator denoted $A$ in order to characterize and compare their 
admissibility and controllability properties. This improves on earlier results in [26], [30], and [38] linking first and second order equations, and in [11], [12], and [13] linking infinite-dimensional equations and their finite-dimensional semi-discretization.

The first main application is the following rough statement concerning the observability in time $T$ by the same general operator $C$ (bounded from $\mathscr{D}(A)$ ):

$$
\begin{array}{lll}
\text { Wave } \ddot{w}+A w=0 \text { for some } T & \Longleftrightarrow \begin{array}{l}
\text { Wave Group } i \dot{v}+\sqrt{A} v=0 \text { for some } T \\
\Downarrow \text { (for any } s>1 \text { ) }
\end{array} \\
\begin{array}{ll}
\ddot{z}+A^{s} z=0, \text { any } T \text { if } \sigma_{\text {ess }}(A)=\emptyset \\
\text { Plates if } s=2
\end{array} & \begin{array}{l}
i \dot{\psi}+(\sqrt{A})^{s} \psi=0, \text { any } T \text { if } \sigma_{\text {ess }}(A)=\emptyset \\
\text { Schrödinger if } s=2
\end{array}
\end{array}
$$

If for example the resolvent of $A$ is compact then the condition $\sigma_{\text {ess }}(A)=\varnothing$ holds (i.e. the spectrum of $A$ is formed of isolated eigenvalues with finite multiplicity). For $s=2$, the implication from waves to plates and the bottom equivalence between plates and Schrödinger are proved in [26], Theorem 5.2, for $C$ bounded on $X$ using the Greiner-Huang-Prüss test (1) hence without explicit constants, therefore without information on the time $T$. Still for $s=2$, the implication from Schrödinger to plates with the same control time $T$ is proved in [42], Theorem 6.8.2, for unbounded $C$, but under an extra eigenvalue condition. The proof by a simple isomorphism with explicit constants of the top equivalence between the wave equation and the wave group in Theorem 3.8 seems to be new. The analysis of the constants in the downward implication from $\sqrt{A}$ to its fractional powers in Theorem 3.5 also seems to be new. Putting these two new facts together proves the downward implication from the wave equation for some $T$ to the plate equation with any $s>0$ and, if $\sigma_{\text {ess }}(A)=\varnothing$, for any $T$. Moreover, it yields this full scale of equivalent resolvent conditions for the observability of the wave equation:

$$
\begin{aligned}
& \exists s>1, M_{s}>0 \text { and } m_{s}>0, \\
& \|x\|_{1}^{2} \leqslant \frac{M_{s}}{\lambda^{2(1-1 / s)}}\left\|\left(A^{s / 2}-\lambda\right) x\right\|_{1}^{2}+m_{s}\|C x\|^{2}, \quad x \in H_{1+s}, \lambda>0,
\end{aligned}
$$

where $H_{s}=\mathscr{D}\left(A^{s / 2}\right)$ with norm $\|u\|_{s}=\left\|A^{s / 2} u\right\|$, with Sobolev-type index $s \in \mathbb{R}$. Corollary 3.9 proves this result and Remark 3.16 provides more background.

The second main application is to obtain filtering scales for the uniform exact observability of the semi-discretization of exactly observable equations. As surveyed in [44], exact controllability may be lost under numerical discretization as the mesh size $h$ tends to zero due to the existence of high-frequency spurious solutions for which the group velocity vanishes.

One of the remedies, called filtering, is to restrict the semi-discretized equation to modes with eigenvalues lower than $\eta / h^{\sigma}$ for some positive $\eta$ and $\sigma$. It is proved in [21] and [27] that $\sigma=2$ is optimal for the boundary observation of one-dimensional 
wave and plates equations with constant coefficients discretized on a uniform mesh. Resolvent conditions where first used in this context by Sylvain Ervedoza to tackle any dimension and non-uniform meshes (cf. [14] for time discretization). In a framework which applies to the finite-element discretization on quasi-uniform shape-regular meshes (cf. Remark 4.1), he obtained in [11] a filtering scale $\sigma$ for the uniform exact observability of approximations of unitary groups with mildly unbounded observation (excluding boundary observation), basically $\sigma=2 / 5$ for interior observation. This was improved by the author into $\sigma=2 / 3$ and published in [12] and [13], cf. Remark 4.15.

Section 4 provides a more general framework in which Theorems 4.11 and 4.18 yield respectively $\sigma=1$ and $\sigma=2 / 3$ for the semi-discretization of interior and boundary observability on shape-regular meshes in the sense of finite elements (for the observation of the Schrödinger equation this improves respectively into $\sigma=4 / 3$ and $\sigma=1$ under the geometric condition of [1], which is always satisfied in one space dimension). Conversely, Theorem 4.12 is a kind of Trotter-Kato theorem deducing admissibility and exact observability of a group from resolvent conditions for its filtered approximations. In this framework, Theorems 4.14 and 4.19 deduce from the uniform exact observability of the filtered approximations that the minimal control provided by the Hilbert Uniqueness Method is the limit of the minimal controls for the filtered approximations. For second order systems, Theorems 4.24 and 4.23 yield respectively $\sigma=4 / 3$ and $\sigma=2 / 3$ for the semi-discretization of interior and boundary observability, improving [12], cf. Remark 4.26. A forthcoming paper compares the approximate observation operator (95) introduced in Theorem 4.23 where $\sigma=2 / 3$ to those in [21], Theorems 3.2 and 3.3, which concern only the simplest system but reach the optimal value $\sigma=2$.

We refer to [6] for a new approximation method for interior control of second order systems with error estimates.

The bottom-line of this paper is to deal with resolvent conditions in the abstract unitary group framework and keep track of the coefficients in the most explicit manner. Some applications to PDEs are briefly given. Many more details and examples are given in [42]. Examples 3.12 and 3.18 seem to be new.

The semiclassical approach to proving these resolvent conditions can be found in [3] and [32]. E.g. Theorem 8 in [3] gives a much simpler proof (based on stationary semiclassical measures) of the boundary control of Schrödinger equation under the sharp geometric condition for the wave equation than the original microlocal proof of [24] (or the proof in [29] based the space-time semiclassical measures). The proof of Theorem 8 in [3] combined with the above resolvent condition for $s=2$ yields the famous results of [1] on the boundary control of the wave equation in a simpler way but without estimate on the control time. Indeed, combining the result of [1] for the wave equation and a control transmutation method similar to [33], Theorem 10.2 in [30] yields more information on the Schrödinger equation (more precisely on the cost of fast controls) than the current resolvent condition approach. 
1.3. Background. The first condition of type (2) was introduced by Kangsheng Liu in Theorem 3.4 in [26] for second-order equations like the wave equation (hence $M$ was replaced by $M / \lambda$ in (2)) under the name frequency domain condition. It was adapted to first-order equations in [46]. Liu used the Greiner-Huang-Prüss test (1) with $\mathcal{E}=i \mathcal{A}-\boldsymbol{C}^{*} \mathcal{C}$ (hence $\boldsymbol{C}$ was replaced by $\boldsymbol{C}^{*} \mathcal{C}$ in (2)) taking advantage of the equivalence between exact controllability and exponential stabilizability. This strategy was limited to observations operator $C$ which are bounded on $X$ and did not give information on the controllability time.

Conditions of type (2) were independently introduced by Nicolas Burq and Maciej Zworski in [3], Theorems 4 and 7, as sufficient to deduce results in the Control Theory of distributed parameter systems from the Spectral Theory of differential operators (one of these theorems is both semiclassical and spectrally localized, both theorems make intricate compatibility assumptions in addition to the resolvent condition). Their direct strategy overcomes both limitations: it allows boundary observation operators and it links the controllability time to the behavior of $M$ for high frequency modes.

The final form of the theorem states that the resolvent condition (2) is both necessary and sufficient for controllability in some time $T$, with explicit relations between the constants $M$ and $m$ in (2) on the one hand, the time $T$, the admissibility constant and the control cost on the other hand (it does not assume the boundedness of $\mathcal{C}$ on $X$ or the compactness of the resolvent of $\mathcal{A}$ ). This theorem and its proof are repeated here as Theorem 2.4 (and e.g. in [42], Theorem 6.6.1) from [30], Theorem 5.1. The proof that the resolvent condition is necessary is close to the proof in [40] of the stronger resolvent condition implied by the stronger assumption of exact observability on $[0, \infty)$. The proof of sufficiency using the Fourier transform is close and was inspired by the proof of Theorem 7 in [3], where the resolvent is assumed compact. This proof shortcuts the use of the Greiner-Huang-Prüss test in [26] and [46].

The analogous result for admissibility is Theorem 2.3 first proved under the additional assumption that the resolvent of $\mathcal{A}$ is compact in [13], Theorem 2.2. The new proof of this Theorem 2.3 shortcuts the use of packets of eigenvectors in [13] and unifies it with the simple proof of Theorem 2.4. From reading [13], Marius Tucsnak proved independently Theorem 2.3, explicit constants excepted, as a direct consequence of older results of George Weiss, cf. Remark 2.8.

We emphasize that, as in the proof of the Greiner-Huang-Prüss test, the key point in the proof that the resolvent condition is sufficient (for both admissibility and exact controllability) is the unitarity of the Fourier transform in Hilbert spaces (Plancherel theorem) used in Lemma 2.7. The two alternative proofs for admissibility in Remark 2.8 both use the Paley-Wiener theorem on the unitarity of the Laplace transform which is also a consequence of the Plancherel theorem.

Alternatively, the Hautus rank condition for finite-dimensional state space may be stated as the following eigenvectors condition discussed in $\$ 2.6$ : for all eigenvector $x$ of $\mathcal{A}, \mathcal{C} \neq 0$. An infinite-dimensional version was introduced in [5] for the exponential stabilizability of unitary groups by a bounded damping perturbation (which is equivalent to the exact observability with bounded observation). It assumes 
that the resolvent of $\mathcal{A}$ is compact and considers clusters of eigenvectors of $\mathcal{A}$ rather than single ones. This version was called wavepackets conditions in [38], where the assumption that $\mathcal{C}$ is bounded has been dropped using the resolvent condition in [30], Theorem 5.1. They are discussed in the more general framework in \$2.5. Sylvain Ervedoza in [13] and [12] introduced another equivalent version of the resolvent condition obtained by optimizing $\lambda$. This paper does not deal with this condition which he called interpolation inequalities.

\section{Resolvent conditions for admissibility and observability}

Most of this paper is about resolvent conditions for the observation of unitary groups. The dual notions of control are recalled in parallel in \$2.1, but they are not used in any statement or proof of $\S 2$ and $\$ 3$. Therefore all considerations of duality could be skipped (i.e. all statements mentioning $X^{\prime}, Y^{\prime}, \mathcal{A}^{\prime}, \mathcal{B}, \xi, A^{\prime}, B, \varphi, \zeta$ ).

2.1. Framework for the control of unitary groups. In this section, we review the general setting for control systems conserving some "energy": admissibility, observability and controllability notions and their duality (cf. [7], [43], and [42]).

Let $X$ and $Y$ be Hilbert spaces. Let $\mathscr{A}: \mathscr{D}(\mathscr{A}) \rightarrow X$ be a self-adjoint operator. Equivalently, $i \mathcal{A}$ generates a strongly continuous group $\left(e^{i t \mathcal{A}}\right)_{t \in \mathbb{R}}$ of unitary operators on $X$. In particular the norm is conserved: $\left\|e^{i t \mathscr{A}} x\right\|=\|x\|, x \in X, t \in \mathbb{R}$. Let $X_{1}$ denote $\mathscr{D}(\mathcal{A})$ with the norm $\|x\|_{1}=\|(\mathcal{A}-\beta) x\|$ for some $\beta \notin \sigma(\mathcal{A})(\sigma(\mathcal{A})$ denotes the spectrum of $\mathcal{A}$, this norm is equivalent to the graph norm and $X_{1}$ is densely and continuously embedded in $X$ ) and let $X_{-1}$ be the completion of $X$ with respect to the norm $\|x\|_{-1}=\left\|(\mathcal{A}-\beta)^{-1} x\right\|$.

Let $X^{\prime}$ be a Hilbert space and $J: X^{\prime} \rightarrow \mathscr{L}(X, \mathbb{C})$ be a conjugate linear Hilbert space isomorphism defined by some pairing $\langle\cdot, \cdot\rangle$ on $X \times X^{\prime}$ which is linear on $X$ and conjugate-linear on $X^{\prime}$, i.e. $(J \xi) x=\langle x, \xi\rangle$ where $\langle\cdot, \cdot\rangle$ is a non-degenerate sesquilinear form such that $|\langle x, \xi\rangle| \leqslant\|x\|\|\xi\|$ and $J$ is onto, $J(\alpha \xi)=\bar{\alpha} J \xi$. From now on, the dual space $\mathscr{L}(X, \mathbb{C})$ of $X$ is identified with $X^{\prime}$ by this pairing. E.g. if this pairing is the inner product of $X$ as in (29), then $X=X^{\prime}$ and this is the Riesz identification; if this pairing is the inner product of a Hilbert space $X_{0}$ in which $X$ is continuously embedded as in (51), then $X^{\prime}$ is the dual of $X$ with respect to the pivot space $X_{0}$, cf. [42], §2.9; similarly, if $X^{\prime}$ is continuously embedded in $X_{0}$, then $X$ is the dual of $X^{\prime}$ with respect to $X_{0}$; in (49) this pairing is not an inner product.

The dual of $\mathcal{A}$ is a self-adjoint operator $\mathcal{A}^{\prime}$ on $X^{\prime}$ (if $J_{R}: X \rightarrow \mathscr{L}(X, \mathbb{C})$ denotes the Riesz isomorphism as in [42], §1.1, then the Hilbert space adjoint of $\mathcal{A}^{\text {is } \mathcal{A}^{*}=}$ $J_{R}^{-1} J \mathcal{A}^{\prime} J^{-1} J_{R}$ ). The dual of $X_{1}$ is the space $X_{-1}^{\prime}$ which is the completion of $X^{\prime}$ with respect to the norm $\|\xi\|_{-1}=\left\|\left(\mathcal{A}^{\prime}-\bar{\beta}\right)^{-1} \xi\right\|$ and the dual of $X_{-1}$ is the space $X_{1}^{\prime}$ which is $\mathscr{D}\left(\mathcal{A}^{\prime}\right)$ with the norm $\|\xi\|_{1}=\left\|\left(\mathcal{A}^{\prime}-\bar{\beta}\right) \xi\right\|$, cf. [42], $\$ 2.10$.

Let $\mathcal{C} \in \mathscr{L}\left(X_{1}, Y\right)$ and let $\mathscr{B} \in \mathscr{L}\left(Y^{\prime}, X_{-1}^{\prime}\right)$ denote its dual. 
We consider the dual observation and control systems with output function $y$ and input function $u$ :

$$
\begin{aligned}
& \dot{x}(t)-i \mathscr{A} x(t)=0, \quad x(0)=x_{0} \in X, \quad y(t)=e x(t), \\
& \dot{\xi}(t)-i \mathcal{A}^{\prime} \xi(t)=\mathscr{B} u(t), \quad \xi(0)=\xi_{0} \in X^{\prime}, \quad u \in L_{\mathrm{loc}}^{2}\left(\mathbb{R} ; Y^{\prime}\right) .
\end{aligned}
$$

The following dual admissibility notions for the observation operator $\varphi$ and the control operator $\mathcal{B}$ are equivalent.

Definition 2.1. The system (3) is admissible if for some time $T>0$ (an thus for any times by the group property) there is an admissibility cost $K_{T}$ such that

$$
\int_{0}^{T}\left\|\mathcal{C} e^{i t \mathcal{A}} x_{0}\right\|^{2} d t \leqslant K_{T}\left\|x_{0}\right\|^{2}, \quad x_{0} \in \mathscr{D}(\mathcal{A}) .
$$

The system (4) is admissible if for some time $T>0$ (an thus for any times) there is an admissibility cost $K_{T}$ such that

$$
\left\|\int_{0}^{T} e^{i t \mathcal{A}^{\prime}} \mathcal{B} u(t) d t\right\|^{2} \leqslant K_{T} \int_{0}^{T}\|u(t)\|^{2} d t, \quad u \in L^{2}\left(\mathbb{R} ; Y^{\prime}\right) .
$$

The admissibility constant in time $T$ is the smallest constant in (5), or equivalently in (6), still denoted $K_{T}$.

Under the admissibility assumption, the output map $x_{0} \mapsto y$ from $\mathscr{D}(\mathcal{A})$ to $L_{\text {loc }}^{2}(\mathbb{R} ; Y)$ has a continuous extension to $X$. The equations (3) and (4) have unique solutions $x \in C(\mathbb{R}, X)$ and $\xi \in C\left(\mathbb{R}, X^{\prime}\right)$ defined by

$$
x(t)=e^{i t \mathscr{A}} x_{0}, \quad \xi(t)=e^{i t \mathcal{A}^{\prime}} \xi(0)+\int_{0}^{t} e^{i(t-s) \mathscr{A}} \mathscr{B} u(s) d s .
$$

The following dual notions of observability and controllability are equivalent.

Definition 2.2. The system (3) is exactly observable in time $T$ at cost $\kappa_{T}$ if the following observation inequality holds:

$$
\left\|x_{0}\right\|^{2} \leqslant \kappa_{T} \int_{0}^{T}\|y(t)\|^{2} d t, \quad x_{0} \in \mathscr{D}(\mathcal{A}) .
$$

The system (4) is exactly controllable in time $T$ at cost $\kappa_{T}$ if for all $\xi_{0}$ in $X^{\prime}$, there is a $u$ in $L^{2}\left(\mathbb{R} ; Y^{\prime}\right)$ such that $u(t)=0$ for $t \notin[0, T], \xi(T)=0$ and

$$
\int_{0}^{T}\|u(t)\|^{2} d t \leqslant \kappa_{T}\left\|\xi_{0}\right\|^{2} .
$$

The controllability cost in time $T$ is the smallest constant in (9), or in (8) and it is still denoted $\kappa_{T}$. 
If the system is exactly controllable then, using the group property, for all $\xi_{0}$ and $\xi_{T}$ in $X^{\prime}$, there is a $u$ in $L^{2}\left(\mathbb{R} ; Y^{\prime}\right)$ such that $\int_{0}^{T}\|u(t)\|^{2} d t \leqslant \kappa_{T}\left\|e^{i T \mathscr{A}^{\prime}} \xi_{0}-\xi_{T}\right\|^{2}$, $u(t)=0$ for $t \notin[0, T]$, and the solution of the system (4) satisfies $\xi(T)=\xi_{T}$.

The assumption $\mathcal{E} \in \mathscr{L}\left(X_{1}, Y\right)$ covers most applications to PDEs but is not really necessary to apply this theory since [43], Remark 3.7, proves that any operator $\mathcal{C}$ with a dense domain $\mathscr{D}(\mathcal{C})$ invariant by $\left(e^{i t \mathcal{A}}\right)_{t \geqslant 0}$ satisfying (5) with $\mathscr{D}(\mathcal{A})$ replaced by $\mathscr{D}(\mathcal{C})$ is in some sense equivalent to an operator in $\mathscr{L}\left(X_{1}, Y\right)$.

2.2. Basic resolvent conditions. In the general framework of $\S 2.1$, we consider the following conditions on $\mathcal{C}$ and $\mathcal{A}$ which are reminiscent of relative boundedness of $\mathcal{C}$ with respect to $\mathcal{A}$ (e.g. [9], Definition 2.1) and resolvent estimates for $\mathcal{A}$ :

$$
\begin{gathered}
\exists L>0, l>0, \forall x \in \mathscr{D}(\mathcal{A}), \quad \lambda \in \mathbb{R},\|\mathcal{C} x\|^{2} \leqslant L\|(\mathcal{A}-\lambda) x\|^{2}+l\|x\|^{2}, \\
\exists M>0, m>0, \forall x \in \mathscr{D}(\mathcal{A}), \quad \lambda \in \mathbb{R},\|x\|^{2} \leqslant M\|(\mathcal{A}-\lambda) x\|^{2}+m\|\mathcal{C} x\|^{2} .
\end{gathered}
$$

The following theorems say that these conditions are necessary and sufficient for admissibility and exact controllability respectively.

Theorem 2.3. The system (3) is admissible if and only if the resolvent condition (10) holds. More precisely, (5) implies (10) with $L=T K_{T}$ and $l=2 K_{T} / T$. Conversely (10) implies (5) with $K_{T}=l T+\sqrt{L l}$.

Theorem 2.4. Assume that the system (3) is admissible. It is exactly observable if and only if the resolvent condition (11) holds. More precisely, (8) implies (11) with $M=T^{2} \kappa_{T} K_{T}$ and $m=2 T \kappa_{T}$. Conversely (11) implies (8) for all $T>\pi \sqrt{M}$ with $\kappa_{T}=2 m T /\left(T^{2}-M \pi^{2}\right)$.

Corollary 2.5. The system (4) is admissible and exactly controllable if and only if $x \mapsto\|(\mathcal{A}-\lambda) x\|+\|\mathcal{C} x\|$ is a norm on $\mathscr{D}(\mathcal{A})$ equivalent to $x \mapsto\|(\mathcal{A}-\lambda) x\|+\|x\|$ uniformly with respect to $\lambda \in \mathbb{R}$.

The proof uses lemmata which do not rely on the assumption that $\mathcal{A}$ is self-adjoint.

Lemma 2.6. For all $T>0, x_{0} \in \mathscr{D}(\mathcal{A}), \lambda \in \mathbb{R}$

$$
\begin{aligned}
& T\left\|\mathcal{C} x_{0}\right\|^{2} \leqslant 2 \int_{0}^{T}\left\|\mathcal{E} e^{i t \mathcal{A}} x_{0}\right\|^{2} d t+T^{2} \int_{0}^{T}\left\|\mathcal{C} e^{i t \mathcal{A}}(\mathcal{A}-\lambda) x_{0}\right\|^{2} d t, \\
& \int_{0}^{T}\left\|\mathcal{C} e^{i t \mathcal{A}} x_{0}\right\|^{2} d t \leqslant 2 T\left\|\mathcal{C} x_{0}\right\|^{2}+T^{2} \int_{0}^{T}\left\|\mathcal{C} e^{i t \mathcal{A}}(\mathcal{A}-\lambda) x_{0}\right\|^{2} d t .
\end{aligned}
$$

Proof. Set $x(t)=e^{i t \mathcal{A}} x_{0}, z(t)=x(t)-e^{i t \lambda} x_{0}$ and $f=i(\mathcal{A}-\lambda) x_{0}$. Since $\dot{x}(t)=$ $i \mathcal{A} x(t)=e^{i t \mathcal{A}}\left(i \lambda x_{0}+f\right)=i \lambda x(t)+e^{i t \mathcal{A}} f$, we have $\dot{z}(t)=i \lambda z(t)+e^{i t \mathcal{A}} f$ and therefore $z(t)=\int_{0}^{t} e^{i(t-s) \lambda} e^{i s \mathcal{A}} f d s$. 
We plug it in $e^{i t \lambda} x_{0}=x(t)-z(t)$ and $x(t)=e^{i t \lambda} x_{0}+z(t)$ to estimate

$$
\begin{aligned}
& \int_{0}^{T}\left|e^{i t \lambda}\right|^{2} d t\left\|e_{0}\right\|^{2} \leqslant 2 \int_{0}^{T}\left\|e_{x}(t)\right\|^{2} d t \\
& +2 \int_{0}^{T} t \int_{0}^{t}\left|e^{i(t-s) \lambda}\right|^{2}\left\|\mathscr{e} e^{i s \mathcal{A}} f\right\|^{2} d s d t, \\
& \int_{0}^{T}\|\operatorname{ex}(t)\|^{2} d t \leqslant 2 \int_{0}^{T}\left|e^{i t \lambda}\right|^{2} d t\left\|e_{0}\right\|^{2} \\
& +2 \int_{0}^{T} t \int_{0}^{t}\left|e^{i(t-s) \lambda}\right|^{2}\left\|C e^{i s \mathcal{A}} f\right\|^{2} d s d t .
\end{aligned}
$$

Since $\lambda \in \mathbb{R}$, we have $\left|e^{i t \lambda}\right|=\left|e^{i(t-s) \lambda}\right|=1$. Now the inequality

$$
\int_{0}^{T} t \int_{0}^{t} F(s) d s d t \leqslant \int_{0}^{T} t \int_{0}^{T} F(s) d s d t=\left(T^{2} / 2\right) \int_{0}^{T} F(s) d s
$$

with $F(s)=\left\|\mathscr{C} e^{i s \mathcal{A}} f\right\|^{2}$ completes the proof of the lemma.

Lemma 2.7. For all Lipschitz function $\chi$ with compact support in $\mathbb{R}, x_{0} \in \mathcal{D}(\mathcal{A})$,

$$
\begin{aligned}
(10) & \Rightarrow \int\left\|\mathcal{E} e^{i t \mathcal{A}} x_{0}\right\|^{2} \chi^{2}(t) d t \leqslant \int\left\|e^{i t \mathcal{A}} x_{0}\right\|^{2}\left(L \dot{\chi}^{2}(t)+l \chi^{2}(t)\right) d t, \\
(11) & \Rightarrow \int\left\|e^{i t \mathscr{A}} x_{0}\right\|^{2}\left(\chi^{2}(t)-M \dot{\chi}^{2}(t)\right) d t \leqslant m \int\left\|\mathcal{C} e^{i t \mathfrak{A}} x_{0}\right\|^{2} \chi^{2}(t) d t,
\end{aligned}
$$

Proof. Let $x_{0} \in \mathscr{D}(\mathcal{A}), x(t)=e^{i t \mathscr{A}} x_{0}, z=\chi x$ and $f=\dot{z}-i \mathscr{A} z$. Since $\dot{x}=i \mathcal{A} x$, we have $f=\dot{\chi} x$. The Fourier transform of $f$ is $\hat{f}(\tau)=-i(\mathcal{A}-\tau) \hat{z}(\tau)$. Applying (10) and (11) with $x=\hat{z}(\tau)$ and $\lambda=\tau$ and integrating with respect to $\tau$ yields

$$
\begin{aligned}
& \int\|\Gamma \hat{z}\|^{2} d \tau \leqslant L \int\|\hat{f}\|^{2} d \tau+l \int\|\hat{z}\|^{2} d \tau, \\
& \int\|\hat{z}\|^{2} d \tau \leqslant m \int\|\Gamma \hat{z}\|^{2} d \tau+M \int\|\hat{f}\|^{2} d \tau .
\end{aligned}
$$

The proof of the lemma is completed by making the following substitutions

$$
\begin{aligned}
\int\|\hat{z}\|^{2} d \tau & =\int\|x\|^{2} \chi^{2} d t, \\
\int\|\hat{f}\|^{2} d \tau & =\int\|x\|^{2} \dot{\chi}^{2} d t, \\
\int\|\varphi \hat{z}\|^{2} d \tau & =\int\|\varphi x\|^{2} \chi^{2} d t,
\end{aligned}
$$


resulting from the unitarity of the Fourier transform, i.e. Plancherel theorem.

Proof of Theorems 2.3 and 2.4. The implications result from Lemma 2.6. The converse results from Lemma 2.7 with the following choices of $\chi$.

To prove the converse in Theorem 2.3, we take $\chi(t)=1$ on $[0, T]$. We have

$$
\int_{0}^{T}\left\|\mathcal{E} e^{i t \mathscr{A}} x_{0}\right\|^{2} d t \leqslant \int\left\|\mathcal{E} e^{i t \mathscr{A}} x_{0}\right\|^{2} \chi^{2}(t) d t
$$

and, since $\left(e^{i t \mathcal{A}}\right)_{t \geqslant 0}$ is a unitary group, the resolvent condition (10) and Lemma 2.7 imply the admissibility inequality (5) with $K_{T}=\int\left(L \dot{\chi}^{2}(t)+l \chi^{2}(t)\right) d t$. Taking the support of $\chi$ equal to $[-\tau, T+\tau]$ and

$$
\chi(T+t)=\chi(-t)=\frac{\sinh (\omega(\tau-t))}{\sinh (\omega \tau)}, \quad t \in[0, \tau],
$$

with $\omega=\sqrt{l / L}$, yields

$$
K_{T}=T l+\sqrt{L l} \frac{\sinh (2 \omega \tau)}{\sinh ^{2}(\omega \tau)} \longrightarrow T l+\sqrt{L l}
$$

as $\tau \rightarrow+\infty$.

To prove the converse in Theorem 2.4, we take $\chi(t)=\varphi(t / T)$ with the support of $\varphi$ equal to $[0,1]$ and $\varphi(t)=\sin (\pi t)$ for $t \in[0,1]$. We have

$$
\int\left\|\mathcal{E} e^{i t \mathcal{A}} x_{0}\right\|^{2} \chi^{2}(t) d t \leqslant\|\varphi\|_{L^{\infty}}^{2} \int_{0}^{T}\left\|\mathcal{C} e^{i t \mathcal{A}} x_{0}\right\|^{2} d t=\int_{0}^{T}\left\|\mathcal{C} e^{i t \mathcal{A}} x_{0}\right\|^{2} d t,
$$

and, since $\left(e^{i t \mathcal{A}}\right)_{t \geqslant 0}$ is a unitary group,

$$
\int\left\|e^{i t \mathscr{A}} x_{0}\right\|^{2}\left(\chi^{2}(t)-M \dot{\chi}^{2}(t)\right) d t=\left\|x_{0}\right\|^{2} I_{T}
$$

with

$$
\begin{aligned}
I_{T} & =\int\left(\varphi^{2}\left(\frac{t}{T}\right)-\frac{M}{T^{2}} \dot{\varphi}^{2}\left(\frac{t}{T}\right)\right) d t \\
& =\frac{\int \varphi^{2}(t) d t}{T}\left(T^{2}-M \frac{\int \dot{\varphi}^{2}(t) d t}{\int \varphi^{2}(t) d t}\right) \\
& =\frac{T^{2}-M \pi^{2}}{2 T} .
\end{aligned}
$$

Thus, for all $T>\sqrt{M} \pi$, (11) and Lemma 2.7 imply (8) with $\kappa_{T}=m / I_{T}$.

Remark 2.8. Concerning the first statement of the admissibility Theorem 2.3 (i.e. without the explicit relation between the constants in (5) and (10)) Marius Tucsnak 
pointed out that the necessity of the resolvent condition (10) results directly from [42], Theorem 4.3.7, and the sufficient from [42], Corollary 5.2.4, with $\alpha=1$. For completeness, we recall Corollary 5.2.4 in [42]: if $\mathcal{E}$ generates a right-invertible semigroup, $\mathscr{C} \in \mathscr{L}(\mathscr{D}(\mathscr{G}), Y), \alpha>\omega_{0}(\mathscr{E})$ and $\left\|\mathscr{C}(\alpha+i \lambda-\mathscr{G})^{-1}\right\|$ is bounded for $\lambda \in \mathbb{R}$ then the system $(\mathcal{G}, \mathcal{C})$ is admissible. Taking $\mathscr{E}=i \mathcal{A}$, since $\mathcal{A}$ is self-adjoint, $\|((1+i \lambda)-\mathcal{G}) x\|^{2}=\|(\lambda-\mathcal{A}) x\|^{2}+\|x\|^{2}$. We also recall that Corollary 5.2.4 in [42] follows from a result of [43], was first explicitly stated in [16], and was given an alternative shorter proof in [45], Theorem 2.2: as pointed out in the introduction, both proofs use the unitarity of the Laplace transform between $L^{2}(0, \infty)$ and the Hardy space $\mathscr{H}^{2}$ on the right half-plane.

\subsection{Resolvent conditions with variable coefficients and restricted spectral in-}

terval. In this subsection, we consider resolvent conditions more general than (10) and (11) in two ways.

Firstly we allow the coefficients to vary (e.g. this is necessary to obtain the characterization for second order systems in $§ 3.2)$ :

$$
\begin{gathered}
\|\mathcal{C} x\|^{2} \leqslant L(\lambda)\|(\mathcal{A}-\lambda) x\|^{2}+l(\lambda)\|x\|^{2}, \quad x \in \mathcal{D}(\mathcal{A}), \lambda \in \mathbb{R}, \\
\|x\|^{2} \leqslant M(\lambda)\|(\mathcal{A}-\lambda) x\|^{2}+m(\lambda)\|\mathcal{C} x\|^{2}, \quad x \in \mathscr{D}(\mathcal{A}), \lambda \in \mathbb{R},
\end{gathered}
$$

where $l, L, m$ and $M$ are locally bounded positive functions on $\mathbb{R}$.

Both (13) and (14) can be easily extended to $\lambda \in \mathbb{C}$ since $\mathcal{A}$ is self-adjoint: $\|(\mathcal{A}-\lambda-i \mu) x\|^{2}=\|(\mathcal{A}-\lambda) x\|^{2}+\mu^{2}\|x\|^{2} \geqslant\|(\mathcal{A}-\lambda) x\|^{2}$ for real $\lambda$ and $\mu$.

Secondly we restrict the interval for the spectral parameter $\lambda$ (many proofs of $\S 3$ rely on this). Recall that $\sigma(\mathcal{A})$ denotes the spectrum of $\mathcal{A}$. Let inf $\mathcal{A}, \sup \mathcal{A}$ and $[\sigma](\mathcal{A})$ denote its infimum, supremum and convex hull (i.e. the smallest interval containing it). E.g. if inf $\mathcal{A}>-\infty$ and $\sup \mathcal{A}=+\infty$ then $[\sigma](\mathcal{A})=[\inf \mathcal{A},+\infty)$. We always assume inf $\mathcal{A} \neq \sup \mathcal{A}$. The following proposition says that there is no loss in restricting (13) and (14) to $\lambda \in[\sigma](\mathcal{A})$ :

$$
\begin{gathered}
\|\mathcal{C} x\|^{2} \leqslant L_{\sigma}(\lambda)\|(\mathcal{A}-\lambda) x\|^{2}+l_{\sigma}(\lambda)\|x\|^{2}, \quad x \in \mathcal{D}(\mathcal{A}), \lambda \in[\sigma](\mathcal{A}), \\
\|x\|^{2} \leqslant M_{\sigma}(\lambda)\|(\mathcal{A}-\lambda) x\|^{2}+m_{\sigma}(\lambda)\|\mathcal{C} x\|^{2}, \quad x \in \mathscr{D}(\mathcal{A}), \lambda \in[\sigma](\mathcal{A}),
\end{gathered}
$$

where $l_{\sigma}, L_{\sigma}, m_{\sigma}$ and $M_{\sigma}$ are locally bounded positive functions on $[\sigma](\mathcal{A})$. This proposition discusses how to extend the functions $l_{\sigma}, L_{\sigma}, m_{\sigma}$ and $M_{\sigma}$ in (15) and (16) into $l, L, m$ and $M$ for which (13) and (14) hold (the converse being obvious).

Proposition 2.9. The restricted resolvent condition (16) implies (14) with functions $(M, m)$ which are the following extensions of $\left(M_{\sigma}, m_{\sigma}\right)$.

(i) If the spectrum of $A$ is bounded from below but not from above then for each $\lambda<\inf \mathcal{A}$ define $(M, m)(\lambda)=\left(M_{\sigma}, m_{\sigma}\right)(2 \inf \mathcal{A}-\lambda)$. If inf $\mathcal{A} \geqslant 0$, sup $\mathcal{A}=$ $+\infty, M_{\sigma}$ and $m_{\sigma}$ are nonincreasing then one may define more simply, for $\lambda \in \mathbb{R}$, $(M, m)(\lambda)=\left(M_{\sigma}, m_{\sigma}\right)(\max \{\inf \mathcal{A},|\lambda|\})$. 
(ii) If the spectrum of $A$ is bounded then for each $\lambda \notin[\sigma](\mathcal{A})$ define $M$ and $m$ as the supremum of $M_{\sigma}$ and the supremum of $m_{\sigma}$ respectively.

(iii) In particular, if $M_{\sigma}$ and $m_{\sigma}$ are constants then $(M, m)=\left(M_{\sigma}, m_{\sigma}\right)$ is always suitable.

With the same extensions of $\left(L_{\sigma}, l_{\sigma}\right)$ into $(L, l),(15)$ implies (13).

Proof. The spectral theorem yields for any non-negative self-adjoint operators $B$ :

$$
\|(B-\mu) x\| \leqslant\|(B+\mu) x\|, \quad x \in \mathscr{D}(B), \mu>0 .
$$

This results from writing $B-\mu=(B+\mu) f(B / \mu)$, where $f(t)=(t-1) /(t+1)$ remains in $[-1,1]$ for $t \in[0, \infty)$. Applying (17) with $\mu=\inf \mathcal{A}-\lambda$ yields

$$
\|(\mathcal{A}-(2 \inf \mathcal{A}-\lambda)) x\| \leqslant\|(\mathcal{A}-\lambda) x\|, \lambda \leqslant \inf \mathcal{A} .
$$

The choice in (i) results from (18). The last sentence in (i) results from 2 inf $\mathcal{A}-\lambda \geqslant$ $\max \{\inf \mathcal{A},-\lambda\}$ for $\lambda \leqslant \inf \mathcal{A}$.

To prove (ii) we assume that both inf $\mathcal{A}$ and $\sup \mathcal{A}$ are finite, (16) holds and we define $M$ and $m$ as the supremum of $M_{\sigma}$ and $m_{\sigma}$ respectively. Thanks to (18), (14) holds for $\lambda \in[2 \inf \mathcal{A}-\sup \mathcal{A}$, sup $\mathcal{A}]$. Applying (17) recursively with $B=$ $\mathcal{A}-(\inf \mathcal{A}-n(\sup \mathcal{A}-\inf \mathcal{A}))$ and $\mu=\inf \mathcal{A}-n(\sup \mathcal{A}-\inf \mathcal{A})-\lambda$ for $n \in \mathbb{N}$ yields that it still holds for $\lambda \in[\inf \mathcal{A}-(n+1)(\sup \mathcal{A}-\inf \mathcal{A}), \sup \mathcal{A}]$ for all $n \in \mathbb{N}$, i.e. for all $\lambda \leqslant \sup \mathcal{A}$. It still holds for all $\lambda \geqslant \sup \mathcal{A}$ by a similar recurrence with $B=\sup \mathcal{A}+n(\sup \mathcal{A}-\inf \mathcal{A})-\mathcal{A}$ and $\mu=\lambda-(\sup \mathcal{A}+n(\sup \mathcal{A}-\inf \mathscr{A}))$.

The following proposition says that there is only a loss of a factor 4 in the main coefficient in restricting (13) and (14) to the sheer spectrum of $\mathcal{A}$ :

$$
\begin{gathered}
\|\mathcal{C} x\|^{2} \leqslant L_{\sigma}(\lambda)\|(\mathcal{A}-\lambda) x\|^{2}+l_{\sigma}(\lambda)\|x\|^{2}, \quad x \in \mathscr{D}(\mathcal{A}), \lambda \in \sigma(\mathcal{A}), \\
\|x\|^{2} \leqslant M_{\sigma}(\lambda)\|(\mathcal{A}-\lambda) x\|^{2}+m_{\sigma}(\lambda)\|\mathcal{C} x\|^{2}, \quad x \in \mathscr{D}(\mathcal{A}), \lambda \in \sigma(\mathcal{A}),
\end{gathered}
$$

where $l_{\sigma}, L_{\sigma}, m_{\sigma}$ and $M_{\sigma}$ are locally bounded positive functions on $\sigma(\mathcal{A})$.

Proposition 2.10. The system (3) is admissible (resp. exactly observable) if and only if the resolvent condition (19) (resp. (20)) holds for some constant $l_{\sigma}$ and $L_{\sigma}$ (resp. for some constant $m_{\sigma}$ and $\left.M_{\sigma}\right)$.

More precisely, (20) implies (14) with functions $(M, m)(\lambda)=\left(4 M_{\sigma}, m_{\sigma}\right)(\pi(\lambda))$ where $\pi(\lambda)$ denotes the spectral value closest to $\lambda$. When the distance of $\lambda$ to the spectrum of $\mathcal{A}$ is large enough this improves into $(M, m)(\lambda)=\left(|\lambda-\pi(\lambda)|^{-2}, 0\right)$. With the same definition of $\left(L_{\sigma}, l_{\sigma}\right)$ from $(L, l)$, (19) implies (13).

Proof. If $\lambda \notin \sigma(\mathcal{A})$, then the spectral theorem yields $\left\|(\mathcal{A}-\lambda)^{-1}\right\| \leqslant|\pi(\lambda)-\lambda|^{-1}$. Hence $\|(\mathscr{A}-\pi(\lambda)) x\| \leqslant\|(\mathscr{A}-\lambda) x\|+\|(\lambda-\pi(\lambda)) x\| \leqslant 2\|(\mathscr{A}-\lambda) x\|$.

The characterization of observability in Proposition 2.10 was proved by contradiction in [46] (for bounded $C$ ) without explicit constants. 
2.4. Resolvent conditions with variable coefficients and the controllability time. From Theorem 2.4 and Proposition 2.9(iii), if the resolvent condition (16) holds with constant $M_{\sigma}$ and $m_{\sigma}$ then exact controllability holds in time $T>\pi \sqrt{M_{\sigma}}$. Hence, if the resolvent condition (16) holds with a smaller coefficient $M_{\sigma}$ on some part of the spectrum then the corresponding part of the system is controllable in a shorter time.

The following proposition ensures that the full system is actually controllable in this shorter time provided the spectral subspace of the complementary part of the spectrum is finite dimensional. Although its statement is slightly different from [42], Proposition 6.4.4, (which does not assume that $\mathcal{A}$ is self-adjoint), its proof is so close to that of Tucsnak and Weiss that it is omitted here. It is based on their earlier simultaneous controllability result in [41], cf. Theorem 6.4.2 in [42].

Proposition 2.11. Assume that the system (3) is admissible and that there is a finite set $S$ of eigenvalues $\lambda$ of $\mathcal{A}$ such that $V_{\lambda}=\operatorname{ker}(\mathcal{A}-\lambda)$ is finite dimensional and all the eigenvectors $x_{\lambda} \in V_{\lambda}$ satisfy $e_{\lambda} \neq 0$.

If exact observability in time $T_{0}>0$ holds for the restricted system

$$
\dot{x}(t)-i \mathscr{A}_{0} x(t)=0, \quad x(0)=x_{0} \in X_{0}, \quad y(t)=e_{x}(t),
$$

where $\mathcal{A}_{0}=\mathbf{1}_{\mathcal{A} \notin S} \mathcal{A}$ is the restriction of $\mathcal{A}$ to the orthogonal space $X_{0}=X_{S}^{\perp}$ of $X_{S}=\mathbf{1}_{\mathcal{A} \in S} X=\bigoplus_{\lambda \in S} V_{\lambda}$ in $X$, then it also holds for the full system (3).

The following propositions improve the basic time estimate in Theorem 2.4. They says roughly that, when computing the control time from $M_{\sigma}$, any compact part of the discrete spectrum can be discarded: in other words, only the essential spectrum matters including $\pm \infty$ when they are limit points of the spectrum.

Proposition 2.12. Assume that the system (3) is admissible and that the resolvent condition (16) holds with a constant coefficient $m_{\sigma}$. From the other coefficient $M_{\sigma}$, define the essential coefficient

$$
M_{\mathrm{ess}}=\inf _{K \Subset \mathbb{R} \backslash \sigma_{\mathrm{ess}}(\mathcal{A})} \sup _{\lambda \in[\sigma(\mathcal{A}) \backslash K]} M_{\sigma}(\lambda),
$$

where $K \Subset \mathbb{R} \backslash \sigma_{\mathrm{ess}}(\mathcal{A})$ means that $K$ is a compact subset of $\mathbb{R}$ which does not intersect the essential spectrum of $\mathcal{A}$.

Then the system (3) is exactly observable for all time $T>\pi \sqrt{M_{\mathrm{ess}}}$.

If $\sigma(\mathcal{A})$ is bounded from below and $\sigma_{\mathrm{ess}}(\mathcal{A})=\emptyset$ then $M_{\mathrm{ess}}=\lim \sup M_{\sigma}(\lambda)$.

Proof. Let $T_{0}>\pi \sqrt{M_{\mathrm{ess}}}$ so that $M_{0}:=\pi^{-2} T_{0}^{2}>M_{\mathrm{ess}}$. By the definition of $M_{\mathrm{ess}}$, there is a $K \Subset \mathbb{R} \backslash \sigma_{\text {ess }}(\mathcal{A})$ such that $\sup _{\lambda \in[\sigma(\mathcal{A}) \backslash K]} M_{\sigma}(\lambda)<M_{0}$.

Since the restriction $\mathcal{A}_{0}=\mathbf{1}_{\mathcal{A}} \notin K \mathscr{A}$ satisfies $\mathscr{D}\left(\mathscr{A}_{0}\right)=\mathscr{D}(\mathscr{A}) \cap X_{0}$ and $\sigma\left(\mathscr{A}_{0}\right)=$ $\sigma(\mathcal{A}) \backslash K$, the resolvent condition (16) and the definition of $K$ imply

$$
\|x\|^{2} \leqslant M_{0}\left\|\left(\mathcal{A}_{0}-\lambda\right) x\right\|^{2}+m_{\sigma}\|\mathcal{C} x\|^{2}, \quad x \in \mathscr{D}\left(\mathscr{A}_{0}\right), \lambda \in[\sigma]\left(\mathscr{A}_{0}\right) .
$$


By Proposition 2.9(iii), this implies that the restricted system in Proposition 2.11 is controllable in time $T_{0}$.

By the definition of $\sigma_{\text {ess }}(\mathcal{A}), K \cap \sigma(\mathcal{A})$ is composed of isolated eigenvalues with finite multiplicities. Since $K$ is compact, the cardinal of $K \cap \sigma(\mathcal{A})$ is finite. Since $m_{\sigma}>0$, the resolvent condition (16) implies $\mathcal{C} x \neq 0$ for any eigenvector $x$ of $\mathcal{A}$.

Applying Proposition 2.11 with $S=K$ completes the proof.

The estimate of the controllability cost in Theorem 2.4 is lost in Proposition 2.12 due to the contradiction argument in the proof of Proposition 2.11. This was the main reason for replacing it with the control transmutation method in [30], §9.

The following version of Proposition 2.12 is better e.g. when $\mathcal{A}$ is neither bounded from below nor from above. The proof is the same except it uses Proposition $2.10 \mathrm{in}-$ stead of Proposition 2.9. A simpler formula for $M_{\mathrm{ess}}$ is also given when the coefficient $M$ in the resolvent condition (11) is continuous. (Note that $\sigma_{\text {ess }}(\mathcal{A})$ is closed.)

Proposition 2.13. Proposition 2.12 still holds if the resolvent condition (16) is replaced by (20) and the definition of the essential coefficient is replaced by

$$
M_{\mathrm{ess}}=4 \inf _{K \in \mathbb{R} \backslash \sigma_{\mathrm{ess}}(\mathcal{A})} \sup _{\lambda \in \sigma(\mathcal{A}) \backslash K} M_{\sigma}(\lambda) .
$$

If $\sigma_{\mathrm{ess}}(\mathcal{A})=\emptyset$ then $M_{\mathrm{ess}}=4 \lim \sup M_{\sigma}(\lambda)$ as $|\lambda| \rightarrow+\infty$ in $\sigma(\mathcal{A})$.

Proposition 2.12 still holds if the resolvent condition (16) is replaced by (11) with continuous $M$ and the definition of the essential coefficient is replaced by

$$
M_{\mathrm{ess}}=\max \left\{\sup _{\lambda \in \sigma_{\mathrm{ess}}(\mathcal{A})} M(\lambda), \limsup _{\lambda \rightarrow-\infty} M(\lambda), \limsup _{\lambda \rightarrow+\infty} M(\lambda)\right\} .
$$

Corollary 2.14. If $\sigma_{\mathrm{ess}}(\mathcal{A})=\emptyset$ (e.g. if the resolvent of $\mathcal{A}$ is compact), the system (3) is admissible and the resolvent condition (20) holds with $m_{\sigma}$ constant and

$$
M_{\sigma}(\lambda) \longrightarrow 0 \text { as }|\lambda| \longrightarrow+\infty, \quad \lambda \in \sigma(\mathcal{A}),
$$

then the system (3) is exactly observable for all times $T>0$.

This corollary is inspired from [3], Theorem 7, which makes more involved assumptions on $(\mathcal{A}, \mathcal{C})$ but allows the coefficient $m$ to vary.

Remark 2.15. Under the additional assumption that $\mathcal{A}$ is bounded from below, Corollary 2.14 says that $M(\lambda) \rightarrow 0$ as $\lambda \rightarrow+\infty$ implies observability for all $T>0$. But observability for all $T>0$ does not imply $M(\lambda) \rightarrow 0$ as $\lambda \rightarrow+\infty$ (Schrödinger equation in a rectangle observed from a strip is a counter-example). What follows says, in a very vague sense: $M(\lambda) \rightarrow 0$ means fast observability of high modes at low $\operatorname{cost} \kappa_{T} \sim \frac{1}{T}$. We refer to [29], Theorem 3.2, for a similar but rigorous statement about fast observability of high modes at low cost. 
Recall the link of $M$ and $m$ to the time $T$, admissibility $K_{T}$ and cost $\kappa_{T}$ :

$T>\pi \sqrt{M}, \kappa_{T}=2 m T /\left(T^{2}-M \pi^{2}\right), \quad$ and $\quad M=T^{2} \kappa_{T} K_{T}, m=2 T \kappa_{T}$.

If the resolvent condition holds with $M(\lambda) \rightarrow 0$ as $\lambda \rightarrow+\infty$, then the restriction of $\mathcal{A}$ to $\mathbf{1}_{|\mathcal{A}|>\lambda} X$ is observable in time $T(\lambda)=\pi \sqrt{2 M(\lambda)}$ at $\operatorname{cost} \kappa_{T(\lambda)}=4 m / T(\lambda)$. This should be considered as "low cost" since $\kappa_{T} \sim \frac{1}{T}$ whenever $\ell$ is the identity, i.e. full observation is available.

Conversely, if the restriction of $\mathcal{A}$ to $\mathbf{1}_{|\mathcal{A}|>\lambda} X$ is observable in time $T(\lambda)$ with $T(\lambda) \rightarrow 0$ and $\gamma=\limsup T(\lambda) \kappa_{T(\lambda)}<\infty$ as $\lambda \rightarrow+\infty$, then the resolvent condition holds with $M(\lambda)<\gamma K_{1} T(\lambda)$ for large $\lambda$. In particular, this implies observability of $\mathcal{A}$ for all $T>0$.

2.5. Wavepackets condition. The wavepackets condition introduced in (H6') in [5] and (1.6) in [38] corresponds to (21) with constant $d$ and $D$. The following proposition generalizes [38], Theorem 1.3: the resolvent of $A$ is not assumed to be compact, the functions in (21) and (14) are not assumed to be constants and their relation is explicit because the proof is direct (does not go through (2.2) in [38]).

Proposition 2.16. The observability resolvent condition (14) implies the wavepackets condition, for any function $d>m$ and associated function $D=\frac{1-m / d}{M}$,

$$
\|x\|^{2} \leqslant d(\lambda)\left\|e_{x}\right\|^{2}, \quad x \in \mathbf{1}_{|\mathcal{A}-\lambda|^{2} \leqslant D(\lambda)} X, \lambda \in \mathbb{R} .
$$

The wavepackets condition (21) and the admissibility resolvent estimate (13) imply the observability resolvent estimate (14) for any function $m>d$ and associated function $M(\lambda)=\delta L+\frac{1+\delta l}{D}$, where $\delta=\left(\frac{1}{d}-\frac{1}{m}\right)^{-1}$. Note that $\delta>d$ and $m=\left(\frac{1}{d}-\frac{1}{\delta}\right)^{-1}$.

Proof. Let $x \in \mathbf{1}_{|\mathcal{A}-\lambda|^{2} \leqslant D(\lambda)} X$. By the spectral theorem $\|(\mathcal{A}-\lambda) x\|^{2} \leqslant D(\lambda)\|x\|^{2}$. Plugging this in (14) yields (21) with $d(\lambda)=\frac{m}{1-D M}$ since $1-D M=\frac{m}{d}>0$.

To prove the converse, we introduce the projection $x_{\lambda}=\mathbf{1}_{|\mathcal{A}-\lambda|^{2} \leqslant D(\lambda)} x$ of $x \in \mathscr{D}(\mathcal{A})$, and $x_{\lambda}^{\perp}=x-x_{\lambda}$. Using $\left\|\boldsymbol{C}_{\lambda}\right\|^{2} \leqslant\left(1+\varepsilon^{2}\right)\|\mathcal{C} x\|^{2}+\left(1+\varepsilon^{-2}\right)\left\|\mathcal{C} x_{\lambda}^{\perp}\right\|^{2}$, $\varepsilon(\lambda)>0$, and applying (13) to estimate this last term, then plugging this in (21) yields

$$
\begin{aligned}
\|x\|^{2} \leqslant & d\left(1+\varepsilon^{2}\right)\|\varphi x\|^{2}+d\left(1+\varepsilon^{-2}\right) L\left\|(\mathcal{A}-\lambda) x_{\lambda}^{\perp}\right\|^{2} \\
& +\left(1+d l\left(1+\varepsilon^{-2}\right)\right)\left\|x_{\lambda}^{\perp}\right\|^{2} .
\end{aligned}
$$

But the spectral theorem implies $\left\|x_{\lambda}^{\perp}\right\|^{2} \leqslant \frac{1}{D}\left\|(\mathcal{A}-\lambda) x_{\lambda}^{\perp}\right\|^{2}$, so that (14) holds with $m=d\left(1+\varepsilon^{2}\right)$ and $M=\left(1+\varepsilon^{-2}\right) d L+\frac{1+d l\left(1+\varepsilon^{-2}\right)}{D}$.

Combining this Proposition 2.16 with Theorem 2.3, yields this restatement of Theorem 2.4 in terms of wavepackets. 
Corollary 2.17. Assume that the system (3) is admissible. It is exactly observable if and only if the following wavepackets condition holds:

$$
\exists D>0, d>0,\|x\| \leqslant \sqrt{d}\|\mathcal{C} x\|, \quad x \in \mathbf{1}_{|\mathcal{A}-\lambda| \leqslant \sqrt{D}} X, \lambda \in \mathbb{R},
$$

More precisely, (8) implies (11) implies (22) with $D=1 /(2 M)=1 /\left(2 T^{2} \kappa_{T} K_{T}\right)$ and $d=2 m=4 T \kappa_{T}$. Conversely (22) implies exact controllability (8) for all $T$ such that $T^{2}>\pi^{2}\left(\frac{1}{D}+d K_{T}\left(T+\frac{2}{D T}\right)\right)$, in particularforall $T>\frac{\pi}{\sqrt{D}}+\pi^{2} d K_{\pi / \sqrt{D}}$. Moreover, (22) implies (8) with the simpler cost formula $\kappa_{T}=\frac{4 d T}{\varepsilon}$ for all $T$ such that $\varepsilon:=T^{2}-\pi^{2}\left(\frac{1}{D}+2 d K_{T}\left(T+\frac{2}{D T}\right)\right)>0$.

Proof. We only make the lengthiest computation: assuming $T>\frac{\pi}{\sqrt{D}}+\pi^{2} d K$, where $K:=K_{\pi / \sqrt{D}}$, we deduce $(T / \pi)^{2}>\frac{1}{D}+d K_{T}\left(T+\frac{2}{D T}\right)$. Since $T \mapsto K_{T}$ is increasing, $T>\frac{\pi}{\sqrt{D}}$ implies $K_{T}>K$ and $\frac{2}{\pi \sqrt{D}}>\frac{2}{D T}$. Hence it suffices to prove that $x:=T / \pi$ satisfies $x^{2}>\frac{1}{D}+d K\left(T+\frac{2}{\pi \sqrt{D}}\right)=: \alpha+2 \beta x$, where $\alpha=\frac{2 d K}{\pi \sqrt{D}}+\frac{1}{D}$ and $\beta=\frac{\pi d K}{2}$. This is equivalent to $x>\beta+\sqrt{\beta^{2}+\alpha}$. Since $\pi>2 / \pi$, this is implied by the assumption which translates into $x>\frac{1}{\sqrt{D}}+\pi d K=$ $\beta+\sqrt{\beta^{2}+\frac{\pi d K}{\sqrt{D}}+\frac{1}{D}}$.

Corollary 2.18. Assume that the system (3) is admissible, that $\sigma_{\mathrm{ess}}(\mathcal{A})=\emptyset$ (e.g. that the resolvent of $\mathcal{A}$ is compact) and that there is a spectral gap $\gamma>0$ in the following sense: $|\lambda-\mu| \geqslant \gamma$ for all distinct eigenvalues $\lambda$ and $\mu$.

The system (3) is exactly observable if and only if the following eigenvectors condition holds:

$$
\exists \delta>0, \quad \text { for all eigenvector } x \text { of } \mathcal{A},\|x\| \leqslant \delta\|\mathcal{C} x\| .
$$

More precisely (23) is equivalent to (22) with $d=\delta^{2}$ and any $D<\gamma^{2} / 4$.

Remark 2.19. Corollary 2.18 slightly generalizes [42], Proposition 8.1.3, in particular eigenvalues of $\mathcal{A}$ need not be simple. It could also be proved by the classical theorem of Ingham on non-harmonic Fourier series, cf. e.g. [42], Theorem 8.1.1, which would give the better time estimate $T>2 \pi / \gamma$. The time estimate in Corollary 2.17 matches Ingham's $T>\pi / \sqrt{D}$ in both asymptotics $D \rightarrow 0$ or $d \rightarrow 0$, whereas the less general Theorem 2.5 in [12] does not, since its time estimate translates into: $T>\frac{2 e}{\sqrt{D}}\left(\frac{\pi}{4} \ln L+\frac{3 \pi}{4}\right)^{1+1 / \ln L}$, with $L=\frac{2 \pi}{3} K_{1 / \sqrt{D}} d \sqrt{D}$.

Example 2.20. Corollary 2.18 applies to the interior observability of the Schrödinger (with $\mathcal{A}=-\Delta_{c}$ ) and wave equations (with $\mathcal{A}=\sqrt{-\Delta_{c}}$, cf. Theorems 3.8 and 3.13) where $\Delta_{c}=\partial_{x}\left(c(x) \partial_{x}\right)$ is the Laplacian with Dirichlet boundary conditions on a segment and $c$ is a positive smooth function. 
Remark 2.21. Consider the Schrödinger equation on a rectangle $[0, a] \times[0,1]$, $a^{2} \notin \mathbb{Q}$, observed from a smaller rectangle $\omega_{x} \times \omega_{y}, \omega_{x} \subset[0, a], \omega_{y} \subset[0,1]$. Although the natural orthonormal basis of eigenfunctions satisfies (23) and although exact controllability does hold (this result due to Jaffard has been extended to any dimension by Komornik, and to partially rectangular billiards in [3], and [4]), Corollary 2.18 (with $\mathcal{A}=-\Delta$ ) does not apply if $a^{2} \notin \mathbb{Q}$, since the gap condition does not hold. It does apply in principle when $a^{2} \in \mathbb{Q}$ (the gap condition holds) but it is not trivial to check (23) since there are eigenspaces with arbitrary large dimension (it is easy in the case of observation from a strip, i.e. $\omega_{y}=[0,1]$, cf. [5], Example 3.a).

2.6. Eigenvectors condition. Although slightly off the topic of this paper, we comment on the following version of the Hautus test in finite dimension already mentioned in $\S 1$ :

$$
\text { for all eigenvector } x \text { of } \mathcal{A}, \quad e x \neq 0 .
$$

When the resolvent of $A$ is compact, Proposition 6.9.1 in [42] proves that it is equivalent to the following observability notion.

Definition 2.22. The system (3) is approximately observable in infinite time if $x=0$ is the only $x \in \mathscr{D}(\mathcal{A})$ such that $y(t)=0$ for all $t \geqslant 0$.

Using the homeomorphism $(i-\mathcal{A})^{-1}: X \rightarrow \mathscr{D}(\mathcal{A})$, this is equivalent to

$$
\bigcap_{t \in \mathbb{R}} \operatorname{ker}\left(\mathscr{C} e^{i t \mathcal{A}}(i-\mathcal{A})^{-1}\right)=\{0\} .
$$

When $\mathcal{C}$ is admissible, this is equivalent to: $x=0$ is the only $x \in X$ such that $y=0$ in $L_{\mathrm{loc}}^{2}(0, \infty)$. This results from considering $x$ as a the limit in $X$ of its usual smooth approximation $x_{\varepsilon} \in \mathscr{D}(\mathcal{A})$ as $\varepsilon \rightarrow 0^{+}$:

$$
\begin{aligned}
x_{\varepsilon} & :=\frac{1}{\varepsilon} \int_{0}^{\varepsilon} e^{i t \mathcal{A}} x d t, \\
y_{\varepsilon}(T) & :=\mathcal{E} e^{i T \mathcal{A}} x_{\varepsilon} \\
& =\frac{1}{\varepsilon} \int_{0}^{\varepsilon} \mathcal{C} e^{i(T+t) \mathcal{A}} x d t \\
& =\int_{T}^{T+\varepsilon} y(t) d t .
\end{aligned}
$$

Proposition 2.23. Let $\mathscr{B}$ denote the $\sigma$-algebra of Borel sets of $\mathbb{R}$. Consider a set $\mathscr{B}_{\sigma} \subset \mathscr{B}$ of bounded sets such that, for any $\Omega \in \mathscr{B}, \Omega \cap \sigma(\mathcal{A})$ is a countable disjoint union of elements of $\mathcal{B}_{\sigma}$ (e.g. if $\mathscr{B}_{\sigma}$ is the set of bounded Borel subset with diameter smaller than $\varepsilon>0$, then any $\Omega \in \mathscr{B}$ can be written $\Omega=\bigsqcup_{k \in \mathbb{Z}} \Omega_{k}$ with $\Omega_{k}=\Omega \cap\left[k \varepsilon,(k+1) \varepsilon\left[\in \mathscr{B}_{\sigma}\right)\right.$. 
The system (3) is approximately observable in infinite time if and only if

$$
\bigcap_{\Omega \in B_{\sigma}} \operatorname{ker}\left(\mathcal{C}_{\mathbf{1}_{\mathcal{A}} \in \Omega}\right)=\{0\} .
$$

In particular, assuming $\sigma_{\mathrm{ess}}(\mathcal{A})=\emptyset$ and defining $\mathscr{B}_{\sigma}$ as the set of singletons $\{\lambda\}$ for all eigenvalues $\lambda$ of $\mathcal{A}$, we obtain that (3) is approximately observable in infinite time if and only if the eigenvector test (24) holds.

Proof. The second of the following equalities results from the spectral theorem:

$$
\begin{aligned}
\bigcap_{t \in \mathbb{R}} \operatorname{ker}\left(\mathcal{C} e^{i t \mathcal{A}}(i-\mathcal{A})^{-1}\right) & =\bigcap_{f \in L^{\infty}(\mathbb{R})} \operatorname{ker}\left(\mathcal{C} f(\mathcal{A})(i-\mathcal{A})^{-1}\right) \\
& =\bigcap_{\Omega \in \mathcal{B}} \operatorname{ker}\left(\boldsymbol{C}_{\mathbf{1}_{\mathcal{A}} \in \Omega}(i-\mathcal{A})^{-1}\right) \\
& =\bigcap_{\Omega \in \mathcal{B}_{\sigma}} \operatorname{ker}\left(\mathcal{C} \mathbf{1}_{\mathcal{A} \in \Omega}(i-\mathcal{A})^{-1}\right) .
\end{aligned}
$$

The first equality (26) results from the Fourier transform in the following way. Consider $x \in \mathscr{D}(\mathcal{A})$, such that $\mathcal{C} e^{i t \mathcal{A}} x=0, t \geqslant 0$. For any $f \in C^{\infty}(\mathbb{R})$ with compact support, $f$ is the Fourier transform of an $\check{f} \in L^{1}(\mathbb{R})$, hence

$$
\varphi f(\mathcal{A}) x=\varphi \int \check{f}(t) e^{-i t \mathcal{A}} x d t=\int \check{f}(t) \varphi e^{-i t \mathcal{A}} x d t=0 .
$$

The property $\mathcal{C} f(\mathcal{A}) x=0$ extends to the set of continuous function $f$ with compact support since $C^{\infty}(\mathbb{R})$ functions with compact support are dense in this set for the $L^{\infty}(\mathbb{R})$ norm (e.g. by convolution). To extend this property to an $f \in L^{\infty}(\mathbb{R})$, first consider a sequence of continuous functions with compact support $\left(f_{n}\right)_{n \in \mathbb{N}}$ bounded in $L^{\infty}(\mathbb{R})$ and converging pointwise to $f$ (e.g. by Lusin theorem). Since $f_{n}(\mathcal{A})$ converges to $f(\mathcal{A})$ pointwise in $X, f_{n}(\mathcal{A}) x$ converges to $f(\mathcal{A}) x$ in $\mathscr{D}(\mathcal{A})$. Hence we still have $\mathscr{C} f(\mathcal{A}) x=0$ for any $f \in L^{\infty}(\mathbb{R})$.

If $\Omega \in \mathscr{B}$ can be written as the disjoint union $\Omega=\bigsqcup_{n \in \mathbb{N}} \Omega_{n}$ with $\Omega_{n} \in \mathscr{B}_{\sigma}$ then $\mathbf{1}_{\mathcal{A} \in \Omega} x=\sum_{n \in \mathbb{N}} \mathbf{1}_{\mathcal{A} \in \Omega_{n}} x$ converges in $X$. Since $\mathscr{C} \in \mathscr{L}\left(X_{1}, Y\right)$, this implies the convergence in $Y$ of $\mathcal{C}_{\mathbf{1}_{\mathcal{A}} \in \Omega}(i-\mathcal{A})^{-1} x=\sum_{n \in \mathbb{N}} \mathcal{C}_{\mathbf{1}_{\mathcal{A} \in \Omega_{n}}(i-\mathcal{A})^{-1} x \text {. Since }}$ $\Omega_{n} \in \mathscr{B}_{\sigma}$ is bounded, $\operatorname{ker}\left(\mathcal{C}_{\mathbf{1}_{\mathcal{A} \in \Omega_{n}}}\right)=\operatorname{ker}\left(\mathcal{C}_{\mathbf{1}_{\mathcal{A}} \in \Omega_{n}}(i-\mathcal{A})^{-1}\right)$. This completes the proof of the last equality in (26).

Proposition 2.23 results from (26) and the equivalent definition (25).

It is not clear that approximate observability in infinite time is an interesting notion for controllability unless the semigroup is analytic. When the semigroup $t \mapsto e^{t \mathcal{A}}$ is analytic, the output $t \mapsto \mathcal{C} e^{t \mathcal{A}} x$ is analytic so that approximate observability in infinite time is equivalent to approximate observability in any time $T>0$, which is equivalent to approximate controllability in any time $T>0$. Nonetheless we mention the following easy implication in our context of unitary group. 
Lemma 2.24. Consider $\Omega \subset \mathbb{R}$ compact and the restriction $\mathcal{A}_{\Omega}$ of $\mathcal{A}$ to the spectral subspace $X_{\Omega}=\mathbf{1}_{\mathcal{A} \in \Omega} X$. If the system (3) is approximately observable in infinite time then the system (4) obtained by replacing $\mathcal{A}$ on $X$ by $\mathcal{A}_{\Omega}$ on $X_{\Omega}$ is approximately observable in any time $T>0$.

Proof. The set $\Omega$ is compact, hence $\mathcal{A}_{\Omega}$ is bounded, hence the semigroup $t \mapsto e^{i t \mathcal{A}_{\Omega}}$ is analytic, hence $y: t \mapsto \mathcal{C} e^{i t \mathcal{A}_{\Omega}} x$ is analytic. By unique continuation, $\left(\mathcal{A}_{\Omega}, \mathcal{C}\right)$ is approximately observable in infinite time if and only if it is approximately observable in any time $T>0$, i.e. $x=0$ is the only $x \in X_{\Omega}$ such that $y(t)=0$ for all $t \in[0, T]$.

2.7. Quasimode condition for the lack of exact controllability. The necessity of resolvent conditions has been widely overlooked as a means to disprove exact observability. There are two more common means. Firstly, to produce an eigenvector which is not observable in the sense that it violates the eigenvector test (24). Secondly, to produce an approximate solution of the system (3) which is close enough to an exact solution and little enough observable so that it violates (11), e.g. the exact observability of the wave equation is disproved in [37] by a space-time Gaussian beams construction (microlocal measures extend this result from hyperbolic to diffractive and gliding geometric rays, cf. [2], and [28]). Here we point out this intermediate means: to produce an almost not observable approximate eigenvector (approximate eigenvectors are also known as quasimode).

In addition to the first order system (3) we shall consider a second order system, anticipating on (48) in section 3.2. For this purpose we use the notations at the beginning of section 3 and consider the second order observation system associated to a self-adjoint, positive and boundedly invertible operator $A$ on a Hilbert space $H_{0}$ with domain $\mathscr{D}(A)$, where $H_{1}$ is $\mathscr{D}(\sqrt{A})$ with the norm $\|x\|_{1}=\|\sqrt{A} x\|_{0}$ :

$$
\ddot{z}(t)+A z(t)=0, \quad z(0)=z_{0} \in H_{1}, \quad \dot{z}(0)=z_{1} \in H_{0}, \quad y(t)=C z(t) .
$$

Definition 2.25. A quasimode for the system (3) at $\lambda \in \overline{\mathbb{R}}$ is a sequence $\left(x_{n}\right)_{n \in \mathbb{N}}$ in $\mathscr{D}(\mathcal{A})$ such that there is a real sequence $\left(\lambda_{n}\right)_{n \in \mathbb{N}}$ satisfying

$$
\left\|x_{n}\right\| \rightarrow 1, \quad\left\|\mathcal{E} x_{n}\right\| \rightarrow 0, \quad\left\|\left(\mathcal{A}-\lambda_{n}\right) x_{n}\right\| \rightarrow 0, \quad \text { and } \quad \lambda_{n} \rightarrow \lambda
$$

as $\quad n \rightarrow \infty$. A quasimode for the second order system (27) is a $\left(x_{n}\right)_{n \in \mathbb{N}}$ in $\mathscr{D}\left(A^{3 / 2}\right)$ such that

$$
\begin{aligned}
& \left\|x_{n}\right\|_{1} \rightarrow 1, \quad\left\|C x_{n}\right\|=o(1), \quad\left\|\left(A-\lambda_{n}\right) x_{\lambda}\right\|_{1}=o\left(\sqrt{\lambda_{n}}\right), \quad \text { and } \quad \lambda_{n} \rightarrow+\infty \\
& \text { as } n \rightarrow \infty .
\end{aligned}
$$

This second definition anticipates on the study of second order systems in $\$ 3.2$ to allow comparison: a quasimode for the wave-like system (27) must only satisfy 
$\left\|(A-\lambda) x_{\lambda}\right\|_{1}=o(\sqrt{\lambda})$ whereas a quasimode for the corresponding Schrödinger-like system (50) with $s=1$ must satisfy the stronger condition $\left\|(A-\lambda) x_{\lambda}\right\|_{1}=o(1)$. The same comparison can be made in the context of interior observation in \$3.2.3.

As a direct consequence of definition 2.25, Theorem 2.4 and Corollary 3.10:

Theorem 2.26. Assume that the system (3) (resp. the second-order system (27)) is admissible. If there is a quasimode for (3) (resp. for (27)) then it is not exactly observable.

Applying Theorem 2.26 to the very large literature on quasimodes provides relevant specific PDEs systems where exact controllability does not hold. We dwell on this quasimode approach in a forthcoming paper, e.g. it deduces from a construction in [35] that the Schrödinger equation is not exactly controllable from the boundary of a domain where the diffusion constant takes some value outside a bounded strictly convex smooth subdomain and a lower value inside this subdomain, with transmission conditions at the boundary of the subdomain.

In keeping with the topic of this paper, we give two rather abstract applications.

Consider two positive self-adjoint operators $A_{1}$ and $A_{2}$ on two Hilbert spaces $H^{1}$ and $H^{2}$. The operator $A_{1} \otimes I+I \otimes A_{2}$ defined on the algebraic tensor product $\mathscr{D}\left(A_{1}\right) \otimes \mathscr{D}\left(A_{2}\right)$ is closable and its closure, denoted $A=A_{1}+A_{2}$ is a positive selfadjoint operator on the closure $H^{1} \bar{\otimes} H^{2}$ of the algebraic tensor products $H^{1} \otimes H^{2}$.

Theorem 2.27. Assume $C_{1} \in \mathscr{L}\left(\mathscr{D}\left(A_{1}\right) ; Y\right), C=C_{1} \otimes I \in \mathscr{L}\left(\mathscr{D}(A) ; Y \bar{\otimes} H^{2}\right)$, and the second order system (27) with $A$ and $C$ is admissible. If $\operatorname{ker} C_{1} \neq\{0\}$ and $A_{2}^{-1}$ is compact then (27) is not exactly observable.

Proof. Since $A_{2}$ has compact resolvent, there is a sequence $\Lambda$ of eigenvalues tending to $+\infty$ and a corresponding sequence $\left(x_{\lambda}^{2}\right)_{\lambda \in \Lambda}$ in $\mathscr{D}\left(A_{2}^{3 / 2}\right)$ of normalized eigenvectors, i.e. $\left(A_{2}-\lambda\right) x_{\lambda}^{2}=0$ and $\left\|x_{\lambda}^{2}\right\|_{1}=1$. Since $\operatorname{ker} C_{1} \neq\{0\}$ and $\mathscr{D}\left(A_{1}^{3 / 2}\right)$ is dense in $\mathscr{D}\left(A_{1}\right)$, there exists $x^{1} \in \mathscr{D}\left(A_{1}^{3 / 2}\right)$ such that $C_{1} x^{1}=0$ and $\left\|x^{1}\right\|_{0}=1$. Now $x_{\lambda}=x^{1} \otimes x_{\lambda}^{2}$ defines a quasimode for (27), since $C x_{\lambda}=C_{1} x^{1} \otimes x_{\lambda}^{2}=0$,

$$
\begin{aligned}
\left\|x_{\lambda}\right\|_{1}^{2} & =\left\|\sqrt{A} x_{\lambda}\right\|_{0}^{2}=\left\langle A x_{\lambda}, x_{\lambda}\right\rangle_{0}=\left\|x^{1}\right\|_{1}^{2}\left\|x_{\lambda}^{2}\right\|_{0}^{2}+\left\|x^{1}\right\|_{0}^{2}\left\|x_{\lambda}^{2}\right\|_{1}^{2} \\
& =\left(\left\|x^{1}\right\|_{1}^{2} / \lambda+\left\|x^{1}\right\|_{0}^{2}\right)\left\|x_{\lambda}^{2}\right\|_{1}^{2}=1+\left\|x^{1}\right\|_{1}^{2} / \lambda \rightarrow 1,
\end{aligned}
$$

and $\left\|(A-\lambda) x_{\lambda}\right\|_{1}^{2}=\left\|A_{1} x^{1} \otimes x_{\lambda}^{2}\right\|_{1}^{2}=\left\|A_{1} x^{1}\right\|_{1}^{2} / \lambda+\left\|A_{1} x^{1}\right\|_{0}^{2}=O(1)=o(\lambda)$.

The same theorem can be stated in the context of interior observation in $\$ 3.2 .3$ and its application to the wave equation was already stated in [26], Theorem 4.5.

In the second application $X=L^{2}\left(\mathbb{R}^{d}\right)$. Assume the self-adjoint operator $\mathcal{A}$ on $X$ is locally compact, i.e. for all compactly supported $\varphi \in C^{\infty}\left(\mathbb{R}^{d}\right)$ considered as a multiplication operator, $\varphi(\mathcal{A}-z)^{-1}$ is compact for some hence all $z \notin \sigma(\mathcal{A})$. Also assume that $\mathcal{A}$ is local, i.e. there exists a non-negative compactly supported 
$\varphi \in C^{\infty}\left(\mathbb{R}^{d}\right)$ such that $\varphi(q)=1$ for $|q| \leqslant 1$ and $\varphi_{n}(q)=\varphi(q / n)$ satisfies $\varphi_{n} \mathscr{D}(\mathcal{A}) \subset \mathscr{D}(\mathcal{A})$ and $\left\|\left(\mathcal{A} \varphi_{n}-\varphi_{n} \mathcal{A}\right)(\mathcal{A}-i)^{-1}\right\| \rightarrow 0$. The observation operator $\mathcal{C} \in \mathscr{L}(\mathscr{D}(\mathcal{A}) ; Y)$ is compactly supported if there is a compact $K \subset \mathbb{R}^{d}$ such that $\mathcal{C}_{x}=0$ for any $x \in \mathscr{D}(\mathcal{A})$ with support in $\mathbb{R}^{d} \backslash K$.

Theorem 2.28. Assume $\mathcal{A}$ is locally compact and local as above. If $\sigma_{\mathrm{ess}}(\mathcal{A}) \neq \varnothing$ then, for all compactly supported $\mathcal{C}$, the system (3) is not exactly observable.

Proof. Let $\lambda \in \sigma_{\text {ess }}(\mathcal{A})$. Equivalently by Theorem 10.6 in [18] there exists a Zhislin sequence $\left(x_{n}\right)_{n \in \mathbb{N}}$ for $A$ and $\lambda$, i.e. $x_{n} \in \mathscr{D}(\mathcal{A}),\left\|x_{n}\right\|=1$, the support of $x_{n}$ is outside the ball of radius $n$ and $\left\|(\mathcal{A}-\lambda) x_{n}\right\| \rightarrow 0$ as $n \rightarrow \infty$. Since $C$ is supported in some ball of radius $n_{0}, C x_{n}=0$ for $n \geqslant n_{0}$. Hence $\left(x_{n}\right)_{n \in \mathbb{N}}$ is a quasimode for the system (3) and Theorem 2.26 completes the proof.

\section{Links between systems with generator build on a positive $A$}

This section investigates the logical links between the control properties of various systems of the form (3) with various positive $\mathcal{A}$ which are defined using the same building block: a positive self-adjoint operator denoted $A$.

The framework for this section is more specific than 2.1 . Let $H_{0}$ and $Y$ be Hilbert spaces with respective norms $\|\cdot\|_{0}$ and $\|\cdot\|$. When the context is unambiguous we shall omit the index 0 in $\|\cdot\|_{0}$. Let $A$ be a self-adjoint, positive and boundedly invertible unbounded operator on $H_{0}$ with domain $\mathscr{D}(A)$.

We introduce the Sobolev scale of spaces based on $A$. For any positive $s$, let $H_{s}$ denote the Hilbert space $\mathscr{D}\left(A^{s / 2}\right)$ with the norm $\|x\|_{s}=\left\|A^{s / 2} x\right\|_{0}$ (which is equivalent to the graph norm $\|x\|_{0}+\left\|A^{s / 2} x\right\|_{0}$ ). We identify $H_{0}$ and $Y$ with their duals with respect to their inner product (i.e. we use them as pivot spaces). Let $H_{-s}$ denote the dual of $H_{s}$. Since $H_{s}$ is densely continuously embedded in $H_{0}$, the pivot space $H_{0}$ is densely continuously embedded in $H_{-s}$, and $H_{-s}$ is the completion of $H_{0}$ with respect to the norm $\|x\|_{-s}=\left\|A^{-s / 2} x\right\|_{0}$. We still denote by $A$ the restriction of $A$ to $H_{S}$ with domain $H_{S+2}$. It is self-adjoint with respect to the $H_{S}$ scalar product. We denote by $A^{\prime}$ its dual with respect to the duality between $H_{s}$ and $H_{-s}$, which is an extension of $A$ to $H_{-s}$ with domain $H_{2-s}$.

Let $C \in \mathscr{L}\left(H_{2} ; Y\right)$ and let $B \in \mathscr{L}\left(Y ; H_{-2}\right)$ denote its dual.

The dual observation and control systems for the generator $A$ are

$$
\begin{array}{ll}
\dot{x}(t)-i A x(t)=0, & x(0)=x_{0} \in H_{0}, \quad y(t)=C x(t), \\
\dot{\xi}(t)-i A^{\prime} \xi(t)=B u(t), & \xi(0)=\xi_{0} \in H_{0}, \quad u \in L_{\mathrm{loc}}^{2}(\mathbb{R} ; Y) .
\end{array}
$$

We consider the following resolvent conditions (which are restricted to the convex 
hull $[\sigma](A)$ of the spectrum of $A$ with variable coefficients $l, L, m$ and $M$ ):

$$
\begin{gathered}
\|C x\|^{2} \leqslant L(\lambda)\|(A-\lambda) x\|_{0}^{2}+l(\lambda)\|x\|_{0}^{2}, \quad x \in \mathcal{D}(A), \lambda \in[\sigma](A), \\
\|x\|_{0}^{2} \leqslant M(\lambda)\|(A-\lambda) x\|_{0}^{2}+m(\lambda)\|C x\|^{2}, \quad x \in \mathcal{D}(A), \lambda \in[\sigma](A),
\end{gathered}
$$

where $l, L, m$ and $M$ are locally bounded positive functions on $[\sigma](A)$.

Example 3.1. We refer to [42], §7.5, for the typical example of the free linear Schrödinger equation on a domain with Dirichlet boundary condition observed from a subset of the domain, or from the Neumann derivative on a subset of the boundary. Systems of such equations can also be written as (28).

\subsection{Systems generated by fractional powers of $\boldsymbol{A}$ and other convex functions} of $\boldsymbol{A}$. For any function $f$ of the form $f(\lambda)=\lambda h(\lambda)$ where $h: \sigma(A) \rightarrow\left[h_{0},+\infty\right)$ is measurable and $h_{0}>0$, the spectral theorem defines a positive self-adjoint operator $f(A)$ such that $\mathscr{D}(f(A)) \subset \mathscr{D}(A)$ and $\sigma(f(A)) \subset f(\sigma(A))$. Therefore we may consider the systems generated by $f(A)$ fitting the general framework of $\$ 2.1$ with $X=H_{0}=X^{\prime}, \beta=0, \mathcal{C}=C$ and $\mathcal{A}=f(A)$ :

$$
\begin{array}{ll}
\dot{x}(t)-i f(A) x(t)=0, & x(0)=x_{0} \in H_{0}, \quad y(t)=C x(t), \\
\dot{\xi}(t)-i f\left(A^{\prime}\right) \xi(t)=B u(t), & \xi(0)=\xi_{0} \in H_{0}, \quad u \in L_{\mathrm{loc}}^{2}\left(\mathbb{R} ; Y^{\prime}\right) .
\end{array}
$$

This section investigates the link between the control properties of (29) and (33).

An example of particular interest is $f(\lambda)=\lambda^{\alpha}$ with $\alpha \geqslant 1$ which defines an operator $f(A)$ known as the fractional power $A^{\alpha}$ of the operator $A$ with domain $\mathscr{D}\left(A^{\alpha}\right)=H_{2 \alpha} \subset \mathscr{D}(A)=H_{2}$ :

$$
\begin{aligned}
\dot{x}(t)-i A^{\alpha} x(t) & =0, \quad x(0)=x_{0} \in H_{0}, \quad y(t)=C x(t), \\
\dot{\xi}(t)-i\left(A^{\prime}\right)^{\alpha} \xi(t) & =B u(t), \quad \xi(0)=\xi_{0} \in H_{0}, \quad u \in L_{\mathrm{loc}}^{2}\left(\mathbb{R} ; Y^{\prime}\right) .
\end{aligned}
$$

In deducing control properties of (33) from (29), convexity is the main property of $f:[0,+\infty) \rightarrow[0,+\infty)$ that our argument relies on. E.g. Theorem 3.2 applies to $f(\lambda)=\lambda \ln (1+\lambda)$ which has an interesting application (cf. [8]). Although systems generated by fractional powers of the Laplacian are a well established modeling tool, we do not know which range of the power-logarithm scale of the Laplacian has ever been actually considered for modeling purposes. Conversely in deducing control properties of (29) from (33), we use homogeneity as an additional property of $f$, therefore Theorem 3.5 only concerns fractional powers.

Throughout this section the norm $\|\cdot\|_{0}$ on the state space $H_{0}$ is simply denoted $\|\cdot\|$ as the norm on the observation space $Y$ without ambiguity. 
Theorem 3.2. If the system (28) for $A$ is admissible (resp. exactly observable) then the system (32) for $f(A)$ is admissible (resp. exactly observable) for any convex function $f:[0,+\infty) \rightarrow[0,+\infty)$ which vanishes only at 0 .

More precisely (31) implies the observability resolvent estimate

$$
\|x\|^{2} \leqslant M_{f}(\lambda)\|(f(A)-\lambda) x\|^{2}+m_{f}(\lambda)\|C x\|^{2}, \quad x \in \mathscr{D}(f(A)), \lambda \in f([\sigma](A)),
$$

with $m_{f}(\lambda)=m\left(f^{-1}(\lambda)\right)$ and $M_{f}(\lambda)=\left(f^{-1}(\lambda) / \lambda\right)^{2} M\left(f^{-1}(\lambda)\right)$. If moreover $f(\lambda)=\lambda h(\lambda)$ then this simplifies as $M_{f}(\lambda)=M\left(f^{-1}(\lambda)\right) / h^{2}\left(f^{-1}(\lambda)\right)$. Similarly (30) implies the admissibility resolvent estimate for $f(A)$ with coefficients defined from $l$ and $L$ as $m_{f}$ and $M_{f}$ were defined from $m$ and $M$.

Note that $f$ is strictly increasing since $0<x<y$ implies $\frac{f(y)-f(x)}{y-x} \geqslant \frac{f(y)}{y}>0$. Moreover convexity implies continuity. Hence $f$ is bijective.

Since $f$ is continuous, the spectrum of $f(A)$ is $\sigma(f(A))=f(\sigma(A))$. Taking convex hulls and using the convexity of $f$ yields $[\sigma](f(A))=[f(\sigma(A))]=f([\sigma](A))$.

Theorem 3.2 results directly from the following simple convexity inequality.

Lemma 3.3. If $f:[0,+\infty) \rightarrow[0,+\infty)$ is convex and vanishes only at 0 then

$$
\|(A-\mu) x\| \leqslant \frac{\mu}{f(\mu)}\|(f(A)-f(\mu)) x\|,
$$

for $x \in \mathscr{D}(f(A))$ and $\mu>0$.

Proof. The hypothesis implies that $f$ is continuous and $t \mapsto t / f(t)$ is positive nonincreasing on $(0,+\infty)$ hence bounded on $[\varepsilon,+\infty)$ for all $\varepsilon>0$. Therefore $f(A)$ is well defined and $\mathscr{D}(f(A)) \subset \mathscr{D}(A)$.

For all $\mu>0$, the difference quotient $g_{\mu}$ is the left continuous function at $t=\mu$ defined on $[0,+\infty)$ by $g_{\mu}(t)=\frac{f(t)-f(\mu)}{t-\mu}$. Since $f$ is convex, $g_{\mu}$ is increasing. Hence $g_{\mu}(t) \geqslant g_{\mu}(0)=\frac{f(\mu)}{\mu}$ for $t \geqslant 0$. Therefore, setting $h_{\mu}:=1 / g_{\mu}$, the spectral theorem yields $\left\|h_{\mu}(A)\right\| \leqslant \frac{\mu}{f(\mu)}$. Now (36) results from $A-\mu=h_{\mu}(A)(f(A)-$ $f(\mu)$ ).

Example 3.4. Theorem 3.2 applies, e.g., when $f(t)=t \log ^{\alpha}(1+t)=t h(t), \alpha \geqslant 1$, and $M$ is a constant. In this case we check that the coefficient $M_{f}$ satisfies:

$$
M_{f}(\lambda)=M / h^{2}\left(f^{-1}(\lambda)\right) \leqslant(1+\alpha)^{2 \alpha} M / \log ^{2 \alpha}(1+\lambda) .
$$

Setting $\lambda=f(\mu)=\mu \log ^{\alpha}(1+\mu)$, we have $1 / h\left(f^{-1}(\lambda)\right)=1 / \log ^{\alpha}(1+\mu)$ and we want to check $1 / \log ^{\alpha}(1+\mu) \leqslant(1+\alpha)^{\alpha} / \log ^{\alpha}(1+\lambda)$, which is equivalent to $1+\lambda \leqslant(1+\mu)^{1+\alpha}$. But this results from $1+\mu^{1+\alpha} \leqslant(1+\mu)^{1+\alpha}$ and this estimate of the logarithm: $\lambda=\mu \log ^{\alpha}(1+\mu) \leqslant \mu^{1+\alpha}$. 
Applying Theorem 3.2 to $f(\lambda)=\lambda^{\alpha}$ with $\alpha>1$ yields

$$
M_{f}(\lambda)=M\left(\lambda^{\frac{1}{\alpha}}\right) / \lambda^{2-2 / \alpha} .
$$

This makes the following notations for the resolvent conditions more convenient when dealing with the fractional powers in (34):

$$
\begin{gathered}
\|C x\|^{2} \leqslant \frac{L_{\alpha}(\lambda)}{\lambda^{2-2 / \alpha}}\left\|\left(A^{\alpha}-\lambda\right) x\right\|^{2}+l_{\alpha}(\lambda)\|x\|^{2}, \quad x \in \mathscr{D}\left(A^{\alpha}\right), \lambda \in[\sigma]\left(A^{\alpha}\right), \\
\|x\|^{2} \leqslant \frac{M_{\alpha}(\lambda)}{\lambda^{2-2 / \alpha}}\left\|\left(A^{\alpha}-\lambda\right) x\right\|^{2}+m_{\alpha}(\lambda)\|C x\|^{2}, \quad x \in \mathscr{D}\left(A^{\alpha}\right), \lambda \in[\sigma]\left(A^{\alpha}\right) .
\end{gathered}
$$

where $l_{\alpha}, L_{\alpha}, m_{\alpha}$ and $M_{\alpha}$ are locally bounded positive functions on $\mathbb{R}$. The first part of the following theorem is the application of Theorem 3.2 with these notations. The new feature is the converse in the second part using the homogeneity of $f$.

Theorem 3.5. If the system (28) for $A$ is admissible (resp. exactly observable) then the system (34) for $A^{\alpha}$ is admissible (resp. exactly observable) for any $\alpha>1$. More precisely (31) implies (38) with $m_{\alpha}(\lambda)=m\left(\lambda^{1 / \alpha}\right)$ and $M_{\alpha}(\lambda)=M\left(\lambda^{1 / \alpha}\right)$. Similarly (30) implies (37) with $l_{\alpha}(\lambda)=l\left(\lambda^{1 / \alpha}\right)$ and $L_{\alpha}(\lambda)=L\left(\lambda^{1 / \alpha}\right)$.

The system (28) for $A$ is admissible if and only if the resolvent condition (37) for $A^{\alpha}$ holds for some $\alpha \geqslant 1$ and some constant $L_{\alpha}$ and $l_{\alpha}$. Assuming that it is admissible, it is exactly observable if and only if the resolvent condition (38) for $A^{\alpha}$ holds for some $\alpha \geqslant 1$ and some constant $M_{\alpha}$ and $m_{\alpha}$ (if moreover $\sigma_{\mathrm{ess}}(A)=\emptyset$ this implies that the system (34) for $A^{\alpha}$ is exactly observable for any positive time).

More precisely, (37) implies (30) with

$$
L(\lambda)=2 \max \left\{\left(2^{\alpha}-1\right)^{2} L_{\alpha}\left(\lambda^{\alpha}\right), 4\|C\|_{\mathscr{L}\left(H_{2} ; Y\right)}^{2}\right\}
$$

and $l(\lambda)=2 l_{\alpha}\left(\lambda^{\alpha}\right)$. Moreover (30) and (38) imply (31) with $m(\lambda)=2 m_{\alpha}\left(\lambda^{\alpha}\right)$ and $M(\lambda)=\max \left\{\left(2^{\alpha}-1\right)^{2} M_{\alpha}\left(\lambda^{\alpha}\right), m(\lambda) L(\lambda)+(1+m(\lambda) l(\lambda)) / \inf A^{2}\right\}$.

Proof. Thanks to Theorem 3.2 and Proposition 2.9 (and Corollary 2.14 for the statement in parenthesis), we only need to prove the last paragraph. We shall prove (30) and (31) by density, taking $x \in \mathscr{D}\left(A^{\alpha}\right)$. Let $\mu \in[\sigma](A)$ and $\varepsilon>0$. In each case we use some spectral projection $x_{\mu}$ of $x$ which depends on $\varepsilon$ and take advantage of:

$$
\begin{gathered}
\|x\|^{2}=\left\|x_{\mu}\right\|^{2}+\left\|x-x_{\mu}\right\|^{2} \\
\|(A-\mu) x\|^{2}=\left\|(A-\mu) x_{\mu}\right\|^{2}+\left\|(A-\mu)\left(x-x_{\mu}\right)\right\|^{2}, \\
\alpha\left\|(A-\mu) x_{\mu}\right\|^{2}+\beta\left\|(A-\mu)\left(x-x_{\mu}\right)\right\|^{2} \leqslant \max \{\alpha, \beta\}\|(A-\mu) x\|^{2} .
\end{gathered}
$$

Since $f(\mu)=\mu^{\alpha}$ satisfies the homogeneity equality $f(t \mu)=t^{\alpha} f(\mu)$, the difference quotient function $g_{\mu}$ defined in Lemma 3.3 satisfies $g_{\mu}(\mu t)=g(t) f(\mu) / \mu=$ $\mu^{\alpha-1} g(t)$ where $g$ is defined on $[0,+\infty)$ by $g(1)=\alpha$ and $g(t)=\left(1-t^{\alpha}\right) /(1-t)$. 
We first assume only the admissibility condition (37) for $A^{\alpha}$. We introduce the projection $x_{\mu}=\mathbf{1}_{A<(1+\varepsilon) \mu} x$ of $x$ on the spectrum of $A$ below $(1+\varepsilon) \mu$. The spectral theorem yields

$$
\begin{aligned}
& \left\|A\left(x-x_{\mu}\right)\right\| \leqslant(1+1 / \varepsilon)\left\|(A-\mu)\left(x-x_{\mu}\right)\right\|, \\
& \frac{1}{\mu^{\alpha-1}}\left\|\left(A^{\alpha}-\mu^{\alpha}\right) x_{\mu}\right\| \leqslant g(1+\varepsilon)\left\|(A-\mu) x_{\mu}\right\| .
\end{aligned}
$$

The former inequality results from writing $A=(A-\mu) h(A / \mu)$ where $h(t)=$ $1 /(1-1 / t)$ is decreasing. The latter inequality results from writing $\mu^{1-\alpha}\left(A^{\alpha}-\mu^{\alpha}\right)=$ $(A-\mu) g(A / \mu)$ where $g$ is increasing. Using $C \in \mathscr{L}\left(H_{2} ; Y\right)$ and (41) yields

$$
\left\|C\left(x-x_{\mu}\right)\right\| \leqslant(1+1 / \varepsilon)\|C\|\left\|(A-\mu)\left(x-x_{\mu}\right)\right\| .
$$

Applying (37) to $x_{\mu}$ and plugging (42) yields

$$
\left\|C x_{\mu}\right\|^{2} \leqslant g^{2}(1+\varepsilon) L_{\alpha}\left(\mu^{\alpha}\right)\left\|(A-\mu) x_{\mu}\right\|^{2}+l_{\alpha}\left(\mu^{\alpha}\right)\left\|x_{\mu}\right\|^{2} .
$$

Plugging (43) and (44) in $\frac{1}{2}\|C x\|^{2} \leqslant\left\|C x_{\mu}\right\|^{2}+\left\|C\left(x-x_{\mu}\right)\right\|^{2}$ and simplifying by (40) yields (30) with

$$
l(\mu)=2 l_{\alpha}\left(\mu^{\alpha}\right) \text { and } L(\mu)=2 \max \left\{g^{2}(1+\varepsilon) L_{\alpha}\left(\mu^{\alpha}\right),(1+1 / \varepsilon)^{2}\|C\|^{2}\right\} .
$$

Taking $\varepsilon=1$ completes the statement that (37) implies (30) in Theorem 3.5.

Now we assume the admissibility condition (30) for $A$ and the observability condition (38) for $A^{\alpha}$. We introduce the projection $x_{\mu}=\mathbf{1}_{A<\mu+\varepsilon} x$ of $x$ on the spectrum of $A$ below $\mu+\varepsilon$. The spectral theorem yields

$$
\begin{gathered}
\left\|x-x_{\mu}\right\| \leqslant \frac{1}{\varepsilon}\left\|(A-\mu)\left(x-x_{\mu}\right)\right\|, \\
\frac{1}{\mu^{\alpha-1}}\left\|\left(A^{\alpha}-\mu^{\alpha}\right) x_{\mu}\right\| \leqslant g(1+\varepsilon / \mu)\left\|(A-\mu) x_{\mu}\right\| .
\end{gathered}
$$

Applying (38) to $x_{\mu}$, plugging (46) in, and using the monotony of $g$ yields

$$
\left\|x_{\mu}\right\|^{2} \leqslant g^{2}(1+\varepsilon / \inf A) M_{\alpha}\left(\mu^{\alpha}\right)\left\|(A-\mu) x_{\mu}\right\|^{2}+m_{\alpha}\left(\mu^{\alpha}\right)\left\|C x_{\mu}\right\|^{2} .
$$

To estimate the last term, we apply (30) to $x-x_{\mu}$ :

$$
\frac{1}{2}\left\|C x_{\mu}\right\|^{2} \leqslant\|C x\|^{2}+L(\mu)\left\|(A-\mu)\left(x-x_{\mu}\right)\right\|^{2}+l(\mu)\left\|x-x_{\mu}\right\|^{2} .
$$

Plugging this in (47), then plugging the resulting inequality in (39), and simplifying by (45) and finally by (40) yields (31) with $m(\mu)=2 m_{\alpha}\left(\mu^{\alpha}\right)$ and

$$
M(\mu)=\max \left\{g^{2}\left(1+\frac{\varepsilon}{\inf A}\right) M_{\alpha}\left(\mu^{\alpha}\right), 2 m_{\alpha}\left(\mu^{\alpha}\right) L(\mu)+\frac{1+2 m_{\alpha}\left(\mu^{\alpha}\right) l(\mu)}{\varepsilon^{2}}\right\} .
$$

Taking $\varepsilon=\inf A$ yields the last statement in Theorem 3.5. 
Remark 3.6. In the particular case of second-order equations, the part of the first sentence concerning exact observability was proved in [46] (without explicit $M_{\alpha}$ and $\left.m_{\alpha}\right)$.

3.2. Second order systems. In this section we start with the general framework for second order systems which suits the boundary control of PDEs. We finish with a framework which suits the interior control of PDEs better.

3.2.1. "Boundary" second order systems. In addition to first order systems for $A$, we consider the dual observation and control second order systems:

$$
\begin{gathered}
\ddot{z}(t)+A z(t)=0, \quad z(0)=z_{0} \in H_{1}, \\
\dot{z}(0)=z_{1} \in H_{0}, \quad y(t)=C z(t), \\
\ddot{\zeta}(t)+A^{\prime} \zeta(t)=B v(t), \quad \zeta(0)=\zeta_{0} \in H_{0}, \\
\dot{\zeta}(0)=\zeta_{1} \in H_{-1}, \quad v \in L_{\mathrm{loc}}^{2}(\mathbb{R} ; Y) .
\end{gathered}
$$

Example 3.7. We refer to [42], §7.4 and 7.6, for the typical example of the wave equation on a domain with Dirichlet boundary condition observed from a subset of the domain, or from the Neumann derivative on a subset of the boundary. We refer to [42], §7.5, for the examples of plate equations.

We shall now explain how they fit in the general framework of $\S 2.1$. The states $x(t)$ and $\xi(t)$ of the systems (48) and (49) at time $t$ and their state spaces $X$ and $X^{\prime}$ are defined by

$$
x(t)=(z(t), \dot{z}(t)) \in X=H_{1} \times H_{0}, \quad \xi(t)=(\zeta(t), \dot{\zeta}(t)) \in X^{\prime}=H_{0} \times H_{-1} .
$$

$X$ is a Hilbert space with the "energy norm" defined by $\left\|\left(z_{0}, z_{1}\right)\right\|^{2}=\left\|\sqrt{A} z_{0}\right\|_{0}^{2}+$ $\left\|z_{1}\right\|_{0}^{2}, X^{\prime}$ is a Hilbert space with norm defined by $\left\|\left(\zeta_{0}, \zeta_{1}\right)\right\|^{2}=\left\|\zeta_{0}\right\|_{0}^{2}+\left\|\zeta_{1}\right\|_{-1}^{2}$, and $X$ is densely continuously embedded in $X^{\prime}$. These spaces are dual with respect to the pairing $\left\langle\left(z_{0}, z_{1}\right),\left(\zeta_{0}, \zeta_{1}\right)\right\rangle=\left\langle A^{1 / 2} z_{0}, A^{-1 / 2} \zeta_{1}\right\rangle_{0}-\left\langle z_{1}, \zeta_{0}\right\rangle_{0}$.

The dual second-order systems (48) and (49) rewrite as dual first order systems (3) and (4), where $u=v, \mathcal{A}$ is defined on the domain $\mathscr{D}(\mathcal{A})=\mathscr{D}(A) \times \mathscr{D}(\sqrt{A})$ by $\mathcal{A}\left(z_{0}, z_{1}\right)=i\left(-z_{1}, A z_{0}\right), \mathcal{A}^{\prime}$ is an extension of $\mathcal{A}$ to $X^{\prime}$ with domain $X, \beta=0, X_{1}$ is $H_{2} \times H_{1}$ with the norm defined by $\left\|\left(z_{0}, z_{1}\right)\right\|_{1}^{2}=\left\|\mathcal{A}\left(z_{0}, z_{1}\right)\right\|^{2}=\left\|\sqrt{A} z_{1}\right\|_{0}^{2}+$ $\left\|A z_{0}\right\|_{0}^{2}$, $\mathcal{C} \in \mathscr{L}\left(X_{1} ; Y\right)$ is defined by $\mathscr{C}\left(z_{0}, z_{1}\right)=C z_{0}$ and $\mathscr{B} \in \mathscr{L}\left(Y ; X_{-1}^{\prime}\right)$ is defined by $\mathscr{B} y=(0, B y)$.

We also consider the dual observation and control first order systems for $A^{s / 2}$, with $s \geqslant 1$ :

$$
\begin{aligned}
& \dot{f}(t)-i A^{s / 2} f(t)=0, \quad f(0)=f_{0} \in H_{1}, \quad y(t)=C f(t), \\
& \dot{\varphi}(t)-i\left(A^{\prime}\right)^{s / 2} \varphi(t)=B u(t), \quad \varphi(0)=\varphi_{0} \in H_{-1}, \quad u \in L_{\mathrm{loc}}^{2}(\mathbb{R} ; Y) .
\end{aligned}
$$


It fits in the general framework of §2.1: $X=H_{1}, X^{\prime}=H_{-1}, \mathcal{C}=C, \mathcal{A}$ is $A^{s / 2}$ with $\mathscr{D}\left(A^{s / 2}\right)=H_{1+s}, \mathcal{A}^{\prime}$ is $\left(A^{\prime}\right)^{s / 2}$ with $\mathscr{D}\left(\left(A^{\prime}\right)^{s / 2}\right)=H_{s-1}, \beta=0$.

We consider the improved resolvent conditions for (50): $\exists L_{s}, l_{s}, M_{s}, m_{s}>0$,

$$
\begin{aligned}
& \|C f\|^{2} \leqslant \frac{L_{s}}{\lambda^{2(1-1 / s)}}\left\|\left(A^{s / 2}-\lambda\right) f\right\|_{1}^{2}+l_{s}\|f\|_{1}^{2}, \quad f \in H_{1+s}, \lambda \in[\sigma]\left(A^{s / 2}\right), \\
& \|f\|_{1}^{2} \leqslant \frac{M_{s}}{\lambda^{2(1-1 / s)}}\left\|\left(A^{s / 2}-\lambda\right) f\right\|_{1}^{2}+m_{s}\|C f\|^{2}, \quad f \in H_{1+s}, \lambda \in[\sigma]\left(A^{s / 2}\right) .
\end{aligned}
$$

Theorem 3.8. The second order system (48) generated by $A$ is admissible (resp. exactly observable) if and only if the first order system (50) with $s=1$ generated by $\sqrt{A}$ is admissible (resp. exactly observable).

More precisely, (10) implies (52) for $s=1$ with $L_{1}=2 L$ and $l_{1}=2 l$; (11) implies (53) for $s=1$ with $M_{1}=M$ and $m_{1}=m / 2$; (52) for $s=1$ implies (10) with $L=L_{1}$ and $l=l_{1}$; (53) for $s=1$ implies (11) with $m=4 m_{1}$ and $M=$ $\max \left\{M_{1}, 2 m_{1}\|C\|_{\mathscr{L}(\mathscr{D}(A) ; Y)}^{2}+1 /(\inf \sqrt{A})^{2}\right\} ;(52)$ and (53) for $s=1$ implies (11) with $m=4 m_{1}$ and $M=\max \left\{M_{1}, 2 m_{1} L_{1}+\left(2 m_{1} l_{1}+1\right) /(\inf \sqrt{A})^{2}\right\}$.

For variable coefficients as in $\$ 2.3$ the result still holds with inf $\sqrt{A}$ replaced by $\lambda+\inf \sqrt{A}$ in the two formulas for $M$. E.g. if $m_{1}=l_{1}$ is constant but $M_{1}=L_{1} \rightarrow 0$ slower than $1 / \lambda^{2}$ as $\lambda \rightarrow+\infty$, then $m$ is constant and $M \rightarrow 0$ as fast as $M_{1}$ in the second formula for $M$ (whereas the first formula does not even ensure $M \rightarrow 0$ ).

Proof. The theorem follows from the Hilbert spaces isomorphism $\mathcal{W}$ from $X=H_{1} \times$ $H_{0}$ onto $W=H_{1} \times H_{1}$ defined by $W\left(z_{0}, z_{1}\right)=\left(z_{0}-i A^{-1 / 2} z_{1}, z_{0}+i A^{-1 / 2} z_{1}\right) / \sqrt{2}$, with inverse $\mathcal{W}^{-1}\left(w_{0}, w_{1}\right)=\left(w_{0}+w_{1}, i \sqrt{A}\left(w_{0}-w_{1}\right)\right) / \sqrt{2}$. Unitarity is easily checked: $\left\|\left(w_{0}, w_{1}\right)\right\|_{W}^{2}:=\left\|w_{0}\right\|_{1}^{2}+\left\|w_{1}\right\|_{1}^{2}=\left(\left\|\sqrt{A} z_{0}-i z_{1}\right\|_{0}^{2}+\| \sqrt{A} z_{0}+\right.$ $\left.i z_{1} \|_{0}^{2}\right) / 2=\left\|\sqrt{A} z_{0}\right\|_{0}^{2}+\left\|-i z_{1}\right\|_{0}^{2}=\left\|\left(z_{0}, z_{1}\right)\right\|^{2}$. The operator $\mathcal{A}$ for the second order system (48) is isomorphic to $\mathcal{A}_{H}=W_{\mathcal{A}} W^{-1}=\sqrt{A}\left(\begin{array}{cc}1 & 0 \\ 0 & -1\end{array}\right)$.

The resolvent conditions (10) and (11) for (48) write: $\forall w_{0}, w_{1} \in H_{2}, \lambda \in$ $[\sigma](\sqrt{A})$,

$$
\begin{aligned}
\frac{1}{2}\left\|C\left(w_{0}+w_{1}\right)\right\|^{2} \leqslant & L\left(\left\|(\sqrt{A}-\lambda) w_{0}\right\|_{1}^{2}+\left\|(\sqrt{A}+\lambda) w_{1}\right\|_{1}^{2}\right) \\
& +l\left(\left\|w_{0}\right\|_{1}^{2}+\left\|w_{1}\right\|_{1}^{2}\right), \\
\left\|w_{0}\right\|_{1}^{2}+\left\|w_{1}\right\|_{1}^{2} \leqslant & M\left(\left\|(\sqrt{A}-\lambda) w_{0}\right\|_{1}^{2}+\left\|(\sqrt{A}+\lambda) w_{1}\right\|_{1}^{2}\right) \\
& +\frac{m}{2}\left\|C\left(w_{0}+w_{1}\right)\right\|^{2} .
\end{aligned}
$$

(Note that the symmetry between $w_{0}$ and $w_{1}$ allows to let $\lambda$ vary only in the positive part of $[\sigma](\mathcal{A})=[\sigma](\sqrt{A}) \cup[\sigma](-\sqrt{A})$.) Taking $w_{1}=0$ proves the first two implications in the second statement of the theorem. 
The converse for admissibility with $L=L_{1}$ and $l=l_{1}$ follows from writing $\frac{1}{2}\left\|C\left(w_{0}+w_{1}\right)\right\|^{2} \leqslant\left\|C w_{0}\right\|^{2}+\left\|C w_{1}\right\|^{2}$ and applying (52) with $s=1$ to $f=w_{0}$ and $f=w_{1}$. To prove the converse for observability, the main step is to apply (53) with $s=1$ to $f=w_{0}$ and write $\left\|C w_{0}\right\|^{2} \leqslant 2\left\|C\left(w_{0}+w_{1}\right)\right\|^{2}+2\left\|C w_{1}\right\|^{2}$. The following two alternative subsidiary steps lead to the alternative values for $M$.

The first value of $M$ results from $\left\|C w_{1}\right\| \leqslant\|C\|\left\|\sqrt{A} w_{1}\right\|_{1}$, and finally simplifying by (inf $\sqrt{A}+\lambda)\left\|w_{1}\right\|_{1} \leqslant\left\|(\sqrt{A}+\lambda) w_{1}\right\|_{1}$.

To prove the second value of $M$, apply (52) with $s=1$ to $f=w_{1}$, change the sign of $\lambda$ thanks to (17), and finally simplify by $\left\|w_{1}\right\|_{1} \leqslant \frac{1}{\inf \sqrt{A}+\lambda}\left\|(\sqrt{A}+\lambda) w_{1}\right\|_{1}$.

3.2.2. Fractional second order systems. We also consider the dual observation and control second order systems for $A^{S}$, with $s \geqslant 1$ :

$$
\begin{gathered}
\ddot{z}(t)+A^{s} z(t)=0, \quad z(0)=z_{0} \in H_{1}, \\
\dot{z}(0)=z_{1} \in H_{1-s}, \quad y(t)=C z(t), \\
\ddot{\zeta}(t)+\left(A^{\prime}\right)^{s} \zeta(t)=B v(t), \quad \zeta(0)=\zeta_{0} \in H_{s-1}, \\
\dot{\zeta}(0)=\zeta_{1} \in H_{-1},
\end{gathered}
$$

They fit the general framework of §2.1: $X=H_{1} \times H_{1-s}, \mathcal{A}\left(z_{0}, z_{1}\right)=i\left(-z_{1}, A^{s} z_{0}\right)$ with the domain $X_{1}=H_{1+s} \times H_{1}, X_{-1}=H_{1-s} \times H_{1-2 s}, X^{\prime}=H_{s-1} \times H_{-1}$, $\left\langle\left(z_{0}, z_{1}\right),\left(\zeta_{0}, \zeta_{1}\right)\right\rangle=\left\langle A^{1 / 2} z_{0}, A^{-1 / 2} \zeta_{1}\right\rangle_{0}-\left\langle A^{(1-s) / 2} z_{1}, A^{(s-1) / 2} \zeta_{0}\right\rangle_{0}$ and therefore $\mathcal{A}^{\prime}\left(\zeta_{0}, \zeta_{1}\right)=i\left(-\zeta_{1},\left(A^{\prime}\right)^{s} \zeta_{0}\right)$ with $X_{1}^{\prime}=H_{2 s-1} \times H_{s-1}, X_{-1}^{\prime}=H_{-1} \times H_{-1-s}$, $\mathcal{C}\left(z_{0}, z_{1}\right)=C z_{0}$ and therefore $\mathcal{B} y=(0, B y)$.

The following corollary of Theorem 3.8 (using Theorem 3.5 with $H_{0}$ and $A$ replaced by $H_{1}$ and $\sqrt{A}$, and using Corollary 2.14 for the time), characterizes the properties of the second order system (48) in terms of improved resolvent conditions for the first order systems (50).

Corollary 3.9. If the second order system (48) is admissible (resp. exactly observable) then for any $s>1$ the first order system (50) and the second order system (54) are admissible (resp. exactly observable, moreover they are exactly observable for any positive time $T$ if $\left.\sigma_{\mathrm{ess}}(A)=\emptyset\right)$.

More precisely, the second order system (48) is admissible if and only if (52) holds for some $s \geqslant 1$. Assuming that it is admissible, it is exactly observable if and only if (53) holds for some $s \geqslant 1$.

The constants $L, l, M, m$ in (10) and (11) for (48) on the one hand, and $L_{s}, l_{s}$, $M_{s}, m_{s}$ in (52) and (53) on the other hand are explicitly related here.

In particular, for $s=2$ we have the following (using Corollary 2.14 for the time)

Corollary 3.10. Admissibility and exact observability for the wave-like equation (48) hold if and only if $\frac{1}{\sqrt{\lambda}}\|(A-\lambda) x\|_{0}+\left\|C A^{-\frac{1}{2}} x\right\|$ is a norm on $\mathscr{D}(A)$ equivalent to 
$\frac{1}{\sqrt{\lambda}}\|(A-\lambda) x\|_{0}+\|x\|_{0}$ uniformly for $\lambda \geqslant \inf A>0$, and it implies admissibility and exact observability (in any positive time $T$ if moreover $\sigma_{\mathrm{ess}}(A)=\emptyset$ ) for the Schrödinger-like equation (50) and the Plate-like equation (54) both with $s=2$.

Example 3.11. When $A$ is the Dirichlet Laplacian and $C$ is the Neumann derivative at the boundary, combining corollary 3.10 with [1] gives another proof of Theorem 4.1 and Proposition 5.1.B in [24]: under the geometric condition of Bardos, Lebeau, and Rauch for the exact observability of the wave equation in $H_{0}^{1} \times L_{2}$ with Neumann observation, exact observability in arbitrary time holds for the Schrödinger equation in $H_{0}^{1}$ with Neumann observation and for the plate equation in $H_{0}^{1} \times H^{-1}$ with Neumann observation. Under the same assumption, the control transmutation method in [30], Theorem 10.2, yields more information on the Schrödinger equation since it provides a geometric bound on the cost of fast controls.

Example 3.12. We now use Theorems 3.8 and 3.2 to interpret in terms of differential operators an initially abstract example due to Thomas Duyckaerts of a positive self-adjoint operator $A_{*}$ with observation $C_{*} \in \mathscr{L}\left(\mathscr{D}\left(A_{*}\right) ; Y\right), Y=\mathbb{C}$, such that the Schrödinger group $e^{i t A_{*}}$ is exactly observable for all positive time but the heat semigroup $e^{-t A_{*}}$ is not final-observable for any time, cf. [8], §5.1. Let $\Delta$ denotes the Laplacian $\partial_{x}^{2}$ on the segment $[0,1]$ with Dirichlet boundary condition, which is negative self-adjoint on $L^{2}(0,1)$ with domain $\mathscr{D}(\Delta)=H_{0}^{1}(0,1) \cap H^{2}(0,1)$. Let $\partial_{\nu}$ denote the derivative at the endpoint 1 . It is well-known that the wave equation $\ddot{w}-\Delta w=\partial_{t}^{2} w-\partial_{x}^{2} w=0$ is observable by the Neumann derivative $\partial_{v}$ in any time $T \geqslant 2$ on the energy space $H_{0}^{1}(0,1) \times L^{2}(0,1)$ (by the unitarity of the discrete Fourier transform known as Parseval's theorem). Hence Theorem 3.8 with $A=-\Delta$ and $s=1$ yields that $\sqrt{-\Delta}$ on $X=L^{2}(0,1)$ is observable by $C=\partial_{\nu}(-\Delta)^{-1 / 2} \in \mathscr{L}\left(H_{1} ; Y\right), Y=\mathbb{C}$. Applying Theorem 3.2 with $A=\sqrt{-\Delta}, f(t)=t \log (1+t)$ and constant $M$ as in example 3.4 yields that $A_{*}:=\sqrt{-\Delta} \log (1+\sqrt{-\Delta})$ satisfies the logarithmically improved resolvent condition:

$$
\|x\|^{2} \leqslant \frac{M_{*}}{\log ^{2}(1+\lambda)}\left\|\left(A_{*}-\lambda\right) x\right\|^{2}+m_{*}\|C x\|^{2}, \quad x \in \mathscr{D}\left(A_{*}\right), \lambda \in \mathbb{R} .
$$

Note that we have $\mathscr{D}\left((-\Delta)^{\varepsilon+1 / 2}\right) \subset \mathscr{D}\left(A_{*}\right) \subset \mathscr{D}(\sqrt{-\Delta})$, for all $\varepsilon>0$ and $C \in$ $\mathscr{L}\left(\mathscr{D}\left(A_{*}\right) ; Y\right)$.

3.2.3. "Interior" second order systems. The previous Theorem 3.8 is adapted to boundary observability (for wave and Schrödinger equations) since $\mathrm{C} \in \mathscr{L}\left(\mathrm{H}_{2} ; \mathrm{Y}\right)$. For interior observability, we have $C \in \mathscr{L}\left(H_{0} ; Y\right)$ and admissibility is obvious. In the following version of Theorem 3.8, the assumption made on the observation operator $C$ is in-between: $C \in \mathscr{L}\left(H_{1} ; Y\right)$, equivalently $B \in \mathscr{L}\left(Y ; H_{-1}\right)$. Accordingly, we 
may consider a larger space of states $\left(z_{0}, z_{1}\right)$ than the previous "energy space":

$$
\begin{gathered}
\ddot{z}(t)+A z(t)=0, \quad z(0)=z_{0} \in H_{0}, \\
\dot{z}(0)=z_{1} \in H_{-1}, \quad y(t)=C z(t), \\
\ddot{\zeta}(t)+A^{\prime} \zeta(t)=B v(t), \quad \zeta(0)=\zeta_{0} \in H_{1}, \\
\dot{\zeta}(0)=\zeta_{1} \in H_{0}, \quad v \in L_{\mathrm{loc}}^{2}(\mathbb{R} ; Y) .
\end{gathered}
$$

It fits in the general framework of §2.1: $X=H_{0} \times H_{-1}$ with norm $\left\|\left(z_{0}, z_{1}\right)\right\|^{2}=$ $\left\|z_{0}\right\|_{0}^{2}+\left\|z_{1}\right\|_{-1}^{2}, X^{\prime}=H_{1} \times H_{0}$ with the "energy norm" $\left\|\left(\zeta_{0}, \zeta_{1}\right)\right\|^{2}=\left\|\zeta_{0}\right\|_{1}^{2}+\left\|\zeta_{1}\right\|_{0}^{2}$, the duality pairing is $\left\langle\left(z_{0}, z_{1}\right),\left(\zeta_{0}, \zeta_{1}\right)\right\rangle=\left\langle z_{0}, \zeta_{1}\right\rangle_{0}-\left\langle A^{-1 / 2} z_{1}, A^{1 / 2} \zeta_{0}\right\rangle_{0}$.

We rewrite the fractional first order systems (34) and (35) with $\alpha=s / 2$ :

$$
\begin{aligned}
& \dot{x}(t)-i A^{s / 2} x(t)=0, \quad x(0)=x_{0} \in H_{0}, \quad y(t)=C x(t), \\
& \dot{\xi}(t)-i\left(A^{\prime}\right)^{s / 2} \xi(t)=B u(t), \quad \xi(0)=\xi_{0} \in H_{0}, \quad u \in L_{\mathrm{loc}}^{2}\left(\mathbb{R} ; Y^{\prime}\right) .
\end{aligned}
$$

We also consider the improved resolvent condition for (58): $\exists L_{s}, l_{s}, M_{s}, m_{s}>0$,

$$
\begin{aligned}
& \|C x\|^{2} \leqslant \frac{L_{s}}{\lambda^{2(1-1 / s)}}\left\|\left(A^{s / 2}-\lambda\right) x\right\|_{0}^{2}+l_{s}\|x\|_{0}^{2}, \quad x \in H_{S}, \lambda \in[\sigma]\left(A^{s / 2}\right), \\
& \|x\|_{0}^{2} \leqslant \frac{M_{s}}{\lambda^{2(1-1 / s)}}\left\|\left(A^{s / 2}-\lambda\right) x\right\|_{0}^{2}+m_{s}\|C x\|^{2} \quad x \in H_{s}, \lambda \in[\sigma]\left(A^{s / 2}\right) .
\end{aligned}
$$

Applying Theorem 3.8 to the observation operator $C A^{1 / 2} \in \mathscr{L}\left(H_{2} ; Y\right)$ and using Theorem 3.5 and Corollary 2.14 (as in Corollary 3.9) yields:

Theorem 3.13. Assume $C \in \mathscr{L}\left(H_{1} ; Y\right)$. The second order system (56) is admissible (resp. exactly observable) if and only if the first order system (58) with $s=1$ is admissible (resp. exactly observable). These imply that, for any $s>1$, (58) is admissible (resp. exactly observable, moreover it is exactly observable for any positive time $T$ if $\left.\sigma_{\text {ess }}(A)=\emptyset\right)$.

Moreover, (56) is admissible if and only if (60) holds for some $s \geqslant 1$. Assuming that it is admissible, it is exactly observable if and only if (61) holds for some $s \geqslant 1$.

The constants $L, l, M, m$ in (10) and (11) for (56) on the one hand, and $L_{s}, l_{s}$, $M_{s}, m_{s}$ on the other hand are explicitly related. In the following example, we only state these relations in the case $s=1$ (e.g. this is used in [8]).

Example 3.14. Assume the two resolvent conditions for $A$ :

$$
\begin{array}{ll}
\|C x\|^{2} \leqslant L_{2}(\lambda)\left(\frac{1}{\lambda}\|(A-\lambda) x\|_{0}^{2}+\|x\|_{0}^{2}\right), & x \in \mathscr{D}(A), \lambda \in[\sigma](A), \\
\|x\|^{2} \leqslant M_{2}(\lambda)\left(\frac{1}{\lambda}\|(A-\lambda) x\|_{0}^{2}+\|C x\|_{0}^{2}\right), & x \in \mathscr{D}(A) . \lambda \in[\sigma](A),
\end{array}
$$


where $L_{2}$ is positively bounded from below and $M_{2} \rightarrow+\infty$ as $\lambda \rightarrow+\infty$. We shall compute the asymptotics as $\lambda \rightarrow+\infty$ of the coefficients in the resolvent conditions (13) and (14) for the wave-like equation (57). Firstly, the converse in Theorem 3.5 with $\alpha=2$ yields

$$
\begin{gathered}
\|C x\|^{2} \leqslant L_{1}(\lambda)\|(\sqrt{A}-\lambda) x\|_{0}^{2}+l_{1}(\lambda)\|x\|_{0}^{2}, \quad x \in \mathscr{D}(\sqrt{A}), \lambda \in[\sigma](\sqrt{A}), \\
\|x\|^{2} \leqslant M_{1}(\lambda)\|(\sqrt{A}-\lambda) x\|_{0}^{2}+m_{1}(\lambda)\|C x\|_{0}^{2}, \quad x \in \mathscr{D}(\sqrt{A}), \lambda \in[\sigma](\sqrt{A}),
\end{gathered}
$$

with $l_{1}(\lambda)=2 L_{2}\left(\lambda^{2}\right), L_{1}(\lambda)=2 \max \left\{9 L_{2}\left(\lambda^{2}\right), 4\|C\|_{\mathscr{L}\left(H_{1} ; Y\right)}^{2}\right\}, m_{1}(\lambda)=2 M_{2}\left(\lambda^{2}\right)$, and $M_{1}(\lambda) \sim 2 M_{2}\left(\lambda^{2}\right) \max \left\{\frac{9}{2}, L_{1}(\lambda)+\frac{l_{1}(\lambda)}{\inf A}\right\}$. Secondly, with the same computations as in Theorem 3.8, Theorem 3.13 yields (13) and (14) with $l(\lambda)=l_{1}(\lambda)=$ $2 L_{2}\left(\lambda^{2}\right), L(\lambda)=L_{1}(\lambda)=2 \max \left\{9 L_{2}\left(\lambda^{2}\right), 4\|C\|_{\mathscr{L}\left(H_{1} ; Y\right)}^{2}\right\}, m(\lambda)=4 m_{1}(\lambda)=$ $8 M_{2}\left(\lambda^{2}\right)$ and $M(\lambda) \sim 2 M_{2}\left(\lambda^{2}\right) \max \left\{\frac{9}{2}, L(\lambda)+\frac{2 L_{2}\left(\lambda^{2}\right)}{\inf A}, 2 L(\lambda)\right\}$. In particular, if $L_{2}$ is a constant, then $L$ is a constant, and $M(\lambda) \sim 2 M_{2}\left(\lambda^{2}\right) \max \left\{\frac{9}{2}, L+\frac{2 L_{2}}{\inf A}, 2 L\right\}$. If $L_{2} \rightarrow+\infty$ as $\lambda \rightarrow+\infty$, then $L(\lambda) \sim 18 L_{2}\left(\lambda^{2}\right)$ and

$$
M(\lambda) \sim 4 L_{2}\left(\lambda^{2}\right) M_{2}\left(\lambda^{2}\right) \max \left\{9+\frac{1}{\inf A}, 18\right\} .
$$

Similarly to Corollary 3.10, we have in the case $s=2$ :

Corollary 3.15. Admissibility and exact observability for the wave-like equation (57) hold if and only if $\frac{1}{\sqrt{\lambda}}\|(A-\lambda) x\|_{0}+\|C x\|$ is a norm on $\mathscr{D}(A)$ equivalent to $\frac{1}{\sqrt{\lambda}}\|(A-\lambda) x\|_{0}+\|x\|_{0}$ uniformly for $\lambda \geqslant \inf A>0$, and it implies admissibility and exact observability (in any positive time $T$ if moreover $\sigma_{\mathrm{ess}}(A)=\emptyset$ ) for the Schrödinger-like equation (29).

Remark 3.16. We first discuss earlier results concerning the first part of Corollary 3.15, i.e. admissibility and observability resolvent conditions on $A$ for the admissibility and the observability of the wave-like equation. The implication for observability was proved in the proof of Theorem 3.4 in [30]. The equivalence under the additional assumption that the resolvent of $A$ is compact was proved in [38], Proposition 4.5, for observability, and in [12], Theorem 2.2, for admissibility by a proof through wavepackets conditions which does not relate explicitly the constants in the resolvent conditions (as partly explained after [12], Theorem 2.2, the submitted version of (2.9) in [12] was (7.2.10) in [11] which contains a spurious term in the right hand side; the privately communicated (52) for $s=2$ gets rid of this spurious term and restricts the spectral interval; it was published as (2.10) in [12]).

Concerning the implication from the wave-like equation to the Schrödinger-like equation in the second part of Corollary 3.15: it was proved in Theorem 3.1 in [30] for observability (cf. [42], Theorem 6.7.2, for a simpler proof under the additional assumption that the resolvent of $\mathcal{A}$ is compact, in which the cost cannot be estimated, cf. Remark 3.17); it was proved in [42], Proposition 6.8.1, for admissibility. 
Remark 3.17. Concerning the implication from the wave-like equation for some time $L>0$ to the Schrödinger-like equation for all times $T>0$ in corollaries 3.10 and 3.15, the following stronger result is proved in [30]: without the assumption $\sigma_{\text {ess }}(A)=\emptyset$, for all times $T>0$, the controllability cost $\kappa_{1, T}$ for the Schrödingerlike equation satisfies $\kappa_{1, T} \leqslant c \exp \left(\alpha L^{2} / T\right)$ where $\alpha$ is a universal constant and the positive constant $c$ depends only on the uniform lower and upper bounds for the ratio between the two norms in Corollaries 3.10 and 3.15 respectively (e.g. in the case of boundary observation, $c$ depends only on $L_{s}, l_{s}, M_{s}, m_{s}$ in (52) and (53) for $s=2$ ). Although it is not explicit in [30], Theorem 3.1, this statement can be easily checked on the explicit bound of $\kappa_{1, T}$ at the end of its proof: $\alpha$ and $\gamma$ come from an independent one dimensional problem, $\kappa$ and $d$ come from [30], Theorem 3.4, hence from [30], Theorem 6.1, hence depend only on the observability constants $M_{2}$ and $m_{2}$ of the wave-like equation, the admissibility constant $K_{1,1}$ of Schrödinger-like equation in time 1 comes from the corresponding constants $L_{2}$ and $l_{2}$ of the wave-like equation. We do not include here the proof of this result since it combines resolvent conditions with the quite different control transmutation method.

Example 3.18. Combining Corollary 3.15 with the interior control version of [1] yields: under the geometric condition of Bardos, Lebeau, and Rauch for the wave equation in $H_{0}^{1} \times L^{2}$ with $L^{2}$ interior controls, exact controllability in arbitrary time holds for the Schrödinger equations generated by fractional Laplacians $(-\Delta)^{\alpha}$, $\alpha>1 / 2$, in $L^{2}$ with $L^{2}$ interior controls. Recall that the heat equation generated by these fractional Laplacians (a.k.a. anomalous diffusion), is null-controllable in any positive time without geometric condition on the control set, cf. e.g. [31].

\section{Semidiscretization of a system with positive $A$}

The framework of this section is the same as the previous section. In particular we keep the notations introduced at the beginning of section 3: a positive $A$, its scale of Sobolev spaces $H_{s}$ and its observation system (28). This section introduces a finite-dimensional approximation of this system which encompasses a wide range of numerical schemes where the state space $H_{0}$ is a space of functions on the continuum $\mathbb{R}^{d}$ discretized on non-uniform meshes. It investigates the links between the infinitedimensional system (28) called the continuous system and such finite-dimensional approximation called semidiscretized system because it applies to the discretization of spatial variables but not the time variable, cf. [14] for time discretization.

4.1. Approximation spaces. Let $\left(V^{h}\right)_{h>0}$ be a family of finite-dimensional vector spaces with injections $J_{h}: V^{h} \rightarrow H_{0}$. We assume that the range $H^{h}:=J^{h} V_{h}$ of the injection $J_{h}$ is included in $\mathscr{D}(\sqrt{A})$, therefore inducing a Hilbert structure on $V^{h}$ from each $H_{s}, s \leqslant 1$. Let $V_{s}^{h}$ denote $V^{h}$ with the corresponding norm $\|v\|_{s}=\left\|J_{h} v\right\|_{s}$, 
$v \in V^{h}$. The dual $J_{h}^{*}: H_{0} \rightarrow V_{0}^{h}$, defines the $H_{0}$-orthogonal projection $J_{h} J_{h}^{*}$ from $H_{0}$ onto $H^{h}$, and the identity operator $J_{h}^{*} J_{h}$ on $V^{h}$.

Let $\pi_{h}$ be the $H_{1}$-orthogonal projection from $H_{1}$ onto $H^{h}$. The only approximation assumption we make is: $\left\|\left(I-\pi_{h}\right) A^{-1 / 2}\right\|_{1}=O(h)$, i.e. $\exists c_{0}>0$,

$$
\left\|x-\pi_{h} x\right\|_{1} \leqslant c_{0} h\|x\|_{2}, \quad x \in \mathscr{D}(A), h>0 .
$$

In other words $\left\|A^{1 / 2} x-A^{1 / 2} \pi_{h} x\right\|_{0} \leqslant c_{0} h\|A x\|_{0}$, recalling $\|x\|_{s}=\left\|A^{s / 2} x\right\|_{0}$.

Only the asymptotics $h \rightarrow 0$ matters in this section, hence $h$ can be restricted to a finite interval $h \in\left(0, h_{0}\right)$. When the approximation space $V^{h}$ is based on a finite element, $h$ is usually the maximal cell diameter of the mesh $h_{K}$, or $h=h_{K}^{\theta}$ for some fixed $\theta>0$.

Remark 4.1. The approximation assumption (62) is satisfied when $J_{h}$ is the canonical injection of the $H_{1}$-conformal approximation space $V^{h}=H^{h}$ based on the $\mathbb{P}_{1}$ Lagrange finite element for a shape-regular family of affine, simplicial, geometrically conformal meshes, cf. e.g. [10], Proposition 1.134, where $J_{h} J_{h}^{*}$ and $\pi_{h}$ are denoted $\Pi_{c, h}^{0,1}$ and $\Pi_{c, h}^{1,1}$, or [36], §3.5, where they are denoted $P_{h}^{1}$ and $\Pi_{1, h}^{1}$. In practice (62) is weaker than (1.9) in [13] where $J_{h} J_{h}^{*}$ and $h$ are denoted $\pi_{h} \pi_{h}^{*}$ and $h^{\theta}$, since (1.9) in [13] is satisfied only for quasi-uniform meshes, cf. e.g. [10], Proposition 1.134(iii), or (3.5.21) in [36]. Quasi-uniform meshes satisfy the inverse inequality (65), cf. [10], Remark 1.143(i), or (6.3.21) in [36], which is not assumed here unless explicitly.

Remark 4.2. The following Lemma 4.4 proves that (62) is equivalent to: $\exists c_{1}>0$,

$$
\inf _{v \in V^{h}}\left(\left\|x-J_{h} v\right\|_{0}+h\left\|x-J_{h} v\right\|_{1}\right) \leqslant c_{1} h^{2}\|x\|_{2}, \quad x \in \mathscr{D}(A), h>0,
$$

or to the existence of an interpolation operator $I_{h}: \mathscr{D}(A) \rightarrow V^{h}$ such that: $\exists c_{1}>0$,

$$
\left\|x-J_{h} I_{h} x\right\|_{0}+h\left\|x-J_{h} I_{h} x\right\|_{1} \leqslant c_{1} h^{2}\|x\|_{2}, \quad x \in \mathscr{D}(A), h>0 .
$$

The approximation assumption appears in the literature in one of these three forms.

Remark 4.3. The framework of this section can be slightly generalized by considering two bounded linear operators: an injection $J_{h}: V^{h} \rightarrow H_{0}$ and an interpolation operator $J_{h}^{*}: H_{0} \rightarrow V^{h}$ (not necessarily the adjoint of $J_{h}$ ) such that $J_{h}^{*} J_{h}$ is the identity operator on $V^{h}$. In this case $J_{h}^{*} J_{h}$ is still a projection from $H_{0}$ onto $H^{h}$ although this projection is not necessarily orthogonal, hence the only failing statements in this section are the first two parts of Lemma 4.8. Moreover, in practice Lemma 4.8 holds anyway since interpolation operators are usually required to satisfy (71), e.g. finite element interpolation operators for a shape-regular family of affine meshes does, cf. e.g. [10], Corollary 1.109. This framework also generalizes to infinite-dimensional Hilbert spaces $\left(V^{h}\right)_{h>0}$. In this case, whenever the admissibility of finite-dimensional systems is used explicitly in a proof, the corresponding statement should assume the admissibility of the semidiscrete system. 
The approximation assumption (62) is the same as (1.7) in [39] (which deals with stabilization rather than observability, and with second rather than first order systems) and (1.4) in [6] where $h$ is denoted $h^{\theta}$. Indeed, each of these papers makes a second approximation assumption: (1.8) in [39] and (1.5) in [6]. They are both a consequence of the first according to the following lemma (which is a simple version of the Aubin-Nitsche lemma, cf. e.g. [10], Lemma 2.31).

Lemma 4.4. The approximation assumption (62) (in $\left.H_{1}\right)$ implies the approximation in $H_{0}:\left\|\left(I-\pi_{h}\right) A^{-1 / 2}\right\|_{\mathscr{L}\left(H_{0}\right)}=O(h)$ and $\left\|\left(I-\pi_{h}\right) A^{-1}\right\|_{\mathscr{L}\left(H_{0}\right)}=O\left(h^{2}\right)$.

More precisely, (62) implies, with the same constant $c_{0}$,

$$
\begin{gathered}
\left\|x-\pi_{h} x\right\|_{0} \leqslant c_{0} h\|x\|_{1}, \quad x \in H_{1}, h>0, \\
\left\|x-\pi_{h} x\right\|_{0} \leqslant c_{0}^{2} h^{2}\|x\|_{2}, \quad x \in H_{2}, h>0 .
\end{gathered}
$$

Proof. Since $A$ and $\pi_{h}$ are selfadjoint on $H_{1}$, the $H_{1}$-adjoint of $\left(I-\pi_{h}\right) A^{-1 / 2}$ is $A^{-1 / 2}\left(I-\pi_{h}\right)$, hence they have the same $H_{1}$ operator norm and its square is the $H_{1}$ operator norm of the latter times the former. Therefore

$$
\begin{aligned}
\left\|\left(I-\pi_{h}\right) A^{-1 / 2}\right\|_{\mathscr{L}\left(H_{0}\right)} & =\left\|A^{-1 / 2}\left(I-\pi_{h}\right)\right\|_{\mathscr{L}\left(H_{1}\right)} \\
& =\left\|\left(I-\pi_{h}\right) A^{-1 / 2}\right\|_{\mathscr{L}\left(H_{1}\right)} \\
& =c_{0} h,
\end{aligned}
$$

asd

$$
\begin{aligned}
\left\|\left(I-\pi_{h}\right) A^{-1}\right\|_{\mathscr{L}\left(H_{0}\right)} & =\left\|\left(I-\pi_{h}\right)^{2} A^{-1}\right\|_{\mathscr{L}\left(H_{0}\right)} \\
& =\left\|A^{-1 / 2}\left(I-\pi_{h}\right)\left(I-\pi_{h}\right) A^{-1 / 2}\right\|_{\mathscr{L}\left(H_{1}\right)}, \\
& =\left\|\left(I-\pi_{h}\right) A^{-1 / 2}\right\|_{\mathscr{L}\left(H_{1}\right)}^{2} \\
& =\left(c_{0} h\right)^{2},
\end{aligned}
$$

where $\left(I-\pi_{h}\right)^{2}=I-\pi_{h}$ since $I-\pi_{h}$ is an $H_{1}$-orthogonal projection as $\pi_{h}$.

4.2. Galerkin approximation of the unitary group. In the framework of $\$ 4.1$, the Ritz-Galerkin variational method considers the finite-dimensional positive selfadjoint operator $G_{h}=\left(\sqrt{A} J_{h}\right)^{*}\left(\sqrt{A} J_{h}\right): V_{h} \rightarrow V_{h}$ and approximates $A$ by the non-negative selfadjoint operator $A_{h}=\left(\sqrt{A} J_{h} J_{h}^{*}\right)^{*}\left(\sqrt{A} J_{h} J_{h}^{*}\right)=J_{h} G_{h} J_{h}^{*}$ on $H_{0}$. Their spectra are related by $\sigma\left(A_{h}\right)=\sigma\left(G_{h}\right) \cup\{0\}$.

Lemma 4.5. The infimum of the spectrum satisfies inf $G_{h} \geqslant \inf A>0$.

The spectrum satisfies $\sigma\left(G_{h}\right) \subset\left[\inf A, \eta_{0} / h^{2}\right]$ if this inverse inequality holds

$$
h\|x\|_{1} \leqslant \sqrt{\eta_{0}}\|x\|_{0}, \quad x \in H^{h} .
$$


Proof. The first inequality results from $(\inf \sqrt{A})^{2}=\inf A$ and

$$
\begin{aligned}
\inf G_{h}=\inf _{v \in V_{h}} \frac{\left\langle G_{h} v, v\right\rangle_{0}}{\|v\|^{2}} & =\inf _{x \in H^{h}} \frac{\langle\sqrt{A} x, \sqrt{A} x\rangle_{0}}{\|x\|_{0}^{2}} \\
& \geqslant \inf _{x \in \mathscr{D}(\sqrt{A})} \frac{\langle\sqrt{A} x, \sqrt{A} x\rangle_{0}}{\|x\|_{0}^{2}} \\
& =(\inf \sqrt{A})^{2} .
\end{aligned}
$$

Using (65):

$$
\sup G_{h}=\sup _{v \in V_{h}}\left\langle G_{h} v, v\right\rangle_{0} /\|v\|^{2}=\sup _{x \in H^{h}}\|x\|_{1}^{2} /\|x\|_{0}^{2} \leqslant \eta_{0} / h^{2} .
$$

The definition of $G_{h}$ implies that the norm in $V_{1}^{h}$ coincides with the " $H_{1}$ Sobolev norm corresponding to $G_{h}$ " and the dual norms also coincide, i.e.

$$
\begin{gathered}
\|v\|_{1}=\left\|J_{h} v\right\|_{1}=\left\|G_{h}^{1 / 2} v\right\|_{0}, \\
\|v\|_{-1}=\left\|J_{h} v\right\|_{-1}=\left\|G_{h}^{-1 / 2} v\right\|_{0}, \quad v \in V^{h} .
\end{gathered}
$$

Lemma 4.6. This equality of bounded operators on $H_{0}$ defines $G_{h}$ in terms of $\pi_{h}$ :

$$
\pi_{h} A^{-1}=J_{h} G_{h}^{-1} J_{h}^{*} .
$$

The approximation assumption (62) is $\left\|\left(A^{-1}-J_{h} G_{h}^{-1} J_{h}^{*}\right) x\right\|_{1} \leqslant c_{0} h\|x\|_{0}, x \in H_{0}$. It implies $A_{h}$ converges to $A$ in $H_{1}$ strongly in the resolvent sense, i.e.

$$
(A-z)^{-1} x \longrightarrow\left(A_{h}-z\right)^{-1} x \text { in } H_{1}, \quad x \in H_{1}, \Im z \neq 0 .
$$

Proof. For all $v \in V^{h},\left\langle\pi_{h} A^{-1} x, J_{h} v\right\rangle_{1}=\left\langle A^{-1} x, J_{h} v\right\rangle_{1}=\left\langle x, J_{h} v\right\rangle_{0}=\left\langle J_{h}^{*} x, v\right\rangle_{0}$ $=\left\langle G_{h}^{-1} J_{h}^{*} x, v\right\rangle_{1}=\left\langle J_{h} G_{h}^{-1} J_{h}^{*} x, J_{h} v\right\rangle_{1}$. This proves (67).

Let $R_{0}(z)=(A-z)^{-1}, z \notin \sigma(A)$, and $R_{h}(z)=J_{h}\left(G_{h}-z\right)^{-1} J_{h}^{*}, z \notin \sigma\left(G_{h}\right)$. According to Lemma 4.5, the distance of 0 to $\sigma(A)$ and to $\sigma\left(G_{h}\right)$ is greater than inf $A$, hence $\left\|R_{h}(0)\right\| \leqslant 1 / \inf A, h \geqslant 0$. For all $z \in \mathbb{C}$ such that $|z|<\inf A$, the Neumann series $R_{h}(z)=\sum_{k=0}^{\infty} z^{k} R_{h}(0)^{k+1}$ converges for all $|z|<\inf A$ and $h \geqslant 0$ and the approximation assumption implies $R_{h}(0) x \rightarrow R_{0}(0) x$ in $H_{1}$ as $h \rightarrow 0$, hence $R_{h}(z) x \rightarrow R_{0}(z) x$ in $H_{1}$. This property is propagated to all $z \in \mathbb{C}$ such that $\Im z \neq 0$ by Neumann series similarly, since the distance of $z$ to $\sigma(A)$ and to $\sigma\left(G_{h}\right)$ is greater than $\Im z$. This completes the proof of the convergence in the strong resolvent sense, since $\sigma\left(A_{h}\right) \subset \mathbb{R}$ and $R_{h}(z)=\left(A_{h}-z\right)^{-1}$ for $z \notin \sigma\left(A_{h}\right)$.

This convergence in the strong resolvent sense is called "convergence in the generalized sense" in [23], §.VIII.1.2, where the following two spectral properties are deduced. All open sets containing a point of $\sigma(A)$ contain at least a point of $\sigma\left(A_{h}\right)$ for sufficiently large $h$, i.e. the spectrum does not expand suddenly in the limit (in 
particular, with Lemma 4.5: $\inf _{h} \inf G_{h}=\inf A$ ). If $\lambda \in \mathbb{R}$ is not an eigenvalue of $A$, then the finite dimensional spectral projections $\mathbf{1}_{A_{h}<\lambda}=J_{h} \mathbf{1}_{G_{h}<\lambda} J_{h}^{*}$ satisfy

$$
\left\|\left(\mathbf{1}_{A<\lambda}-\mathbf{1}_{A_{h}<\lambda}\right) x\right\|_{1} \longrightarrow 0, \quad x \in H_{1} .
$$

The following theorem is a quantitative version of the semigroup approximation result in [23], Theorem IX.2.16, i.e. convergence in the strong resolvent sense implies strong convergence of the generated semigroups uniformly on finite time intervals.

Theorem 4.7. If $x^{h} \rightarrow x$ in $H_{0}$ (resp. in $H_{1}$ ) then $e^{i t A_{h}} x^{h} \rightarrow e^{i t A} x$ in $H_{0}$ (resp. in $H_{1}$ ) uniformly on finite intervals of $t$.

More precisely, (62) implies these convergence rates in $H_{1}$ and $H_{0}$ :

$$
\begin{gathered}
\left\|\left(e^{i t A_{h}}-e^{i t A}\right) x\right\|_{1} \leqslant c_{0} h\left(t\|x\|_{4}+2\|x\|_{2}\right), \quad x \in H_{4}, h>0, t \in \mathbb{R}, \\
\left\|\left(e^{i t A_{h}}-e^{i t A}\right) x\right\|_{0} \leqslant c_{0}^{2} h^{2}\left(t\|x\|_{4}+2\|x\|_{2}\right), \quad x \in H_{4}, h>0, t \in \mathbb{R} .
\end{gathered}
$$

Proof. We recall (IX.2.27) in [23, ] which is simply verified by taking time derivatives: with $R(\varepsilon)=(\varepsilon+A)^{-1}$ and $R_{h}(\varepsilon)=\left(\varepsilon+A_{h}\right)^{-1}$,

$$
R_{h}(\varepsilon)\left(e^{i t A_{h}}-e^{i t A}\right) R(\varepsilon)=i \int_{0}^{t} e^{i(t-s) A_{h}}\left(R(\varepsilon)-R_{h}(\varepsilon)\right) e^{i s A} d s .
$$

Taking the limit $\varepsilon \rightarrow 0$ yields, since $R_{h}(\varepsilon) \rightarrow J_{h} G_{h}^{-1} J_{h}^{*}=\pi_{h} A^{-1}$ due to (67), and since $R_{h}(\varepsilon) e^{i t A_{h}}=e^{i t A_{h}} R_{h}(\varepsilon)$ :

$$
\left(e^{i t A_{h}} \pi_{h}-\pi_{h} e^{i t A}\right) A^{-2}=i \int_{0}^{t} e^{i(t-s) A_{h}}\left(I-\pi_{h}\right) A^{-1} e^{i s A} d s .
$$

Combining this with (62), (64) and the unitarity of the group first yields

$$
\begin{gathered}
\left\|\left(e^{i t A_{h}} \pi_{h}-\pi_{h} e^{i t A}\right) x\right\|_{1} \leqslant c_{0} h t\|x\|_{4}, \quad x \in H_{4}, h>0, t \in \mathbb{R}, \\
\left\|\left(e^{i t A_{h}} \pi_{h}-\pi_{h} e^{i t A}\right) x\right\|_{0} \leqslant c_{0}^{2} h^{2} t\|x\|_{4}, \quad x \in H_{4}, h>0, t \in \mathbb{R},
\end{gathered}
$$

then completes the proof of (69) and (70). These imply the first statement of the theorem since $H_{4}=D\left(A^{2}\right)$ is dense in $H_{1}$ and in $H_{0}$, and the group is unitary.

The following lemma is used to approximate initial data in Theorems 4.14 and 4.19.

Lemma 4.8. The approximation assumption (62) implies

$$
\begin{gathered}
\left\|x-J_{h} J_{h}^{*} x\right\|_{0} \leqslant c_{0} h\|x\|_{1}, \quad x \in H_{1}, h>0, \\
\left\|x-J_{h} J_{h}^{*} x\right\|_{-1} \leqslant c_{0} h\|x\|_{0}, \quad x \in H_{0}, h>0, \\
\left\|x-J_{h} J_{h}^{*} x\right\|_{-1} \leqslant c_{0}^{2} h^{2}\|x\|_{1}, \quad x \in H_{1}, h>0,
\end{gathered}
$$

$J_{h} J_{h}^{*} x \rightarrow x$ in $H_{0}$ for all $x \in H_{0}$ and $A_{h} \pi_{h} A^{-1} x \rightarrow x$ in $H_{-1}$ for all $x \in H_{-1}$. 
Proof. Using that $J_{h} J_{h}^{*}$ hence $I-J_{h} J_{h}^{*}$ are $H_{0}$-orthogonal projection, $\left\|J_{h} J_{h}^{*} \xi\right\|_{0} \leqslant$ $\|\xi\|_{0}, \xi \in H_{0}$, and $\left\|x-J_{h} J_{h}^{*} x\right\|_{0} \leqslant\left\|x-\pi_{h} x\right\|_{0}, x \in H_{1}$, Hence (62) implies (71). Replacing $\pi_{h}$ by $J_{h} J_{h}^{*}$ and $H_{1}$ by $H_{0}$ in the proof of Lemma 4.4 deduces (72), (73).

To prove the first convergence, let $x \in H_{0}$ and $\varepsilon>0$. There exists $x_{\varepsilon} \in H_{1}$ such that $\left\|x-x_{\varepsilon}\right\|_{0} \leqslant \varepsilon$. Taking $\xi=x-x_{\varepsilon}$ yields $\left\|J_{h} J_{h}^{*}\left(x-x_{\varepsilon}\right)\right\|_{0} \leqslant \varepsilon$ and (71) yields $\left\|x_{\varepsilon}-J_{h} J_{h}^{*} x_{\varepsilon}\right\|_{0} \leqslant c_{0} h\left\|x_{\varepsilon}\right\|_{1}$. Hence $\left\|x-J_{h} J_{h}^{*} x\right\|_{0} \leqslant 2 \varepsilon+c_{0} h\left\|x_{\varepsilon}\right\|_{1}$. Taking first the limsup as $h \rightarrow 0$ then the limit $\varepsilon \rightarrow 0$ completes the proof of the first convergence.

The second convergence is proved similarly by density of $H_{0}$ in $H_{-1}$ since, for $x_{\varepsilon} \in H_{0}, A_{h} \pi_{h} A^{-1} x_{\varepsilon}=J_{h} G_{h} J_{h}^{*} J_{h} G_{h}^{-1} J_{h}^{*} x_{\varepsilon}=J_{h} J_{h}^{*} x_{\varepsilon}$, and, for $\xi=x-x_{\varepsilon} \in$ $H_{-1},\left\|A_{h} \pi_{h} A^{-1} \xi\right\|_{-1}=\left\|G_{h} J_{h}^{*} \pi_{h} A^{-1} \xi\right\|_{-1}=\left\|J_{h}^{*} \pi_{h} A^{-1} \xi\right\|_{1}=\left\|\pi_{h} A^{-1} \xi\right\|_{1} \leqslant$ $\left\|A^{-1} \xi\right\|_{1}=\|\xi\|_{-1}$ using (67), (66), and that $\pi_{h}$ is an $H_{1}$-orthogonal projection .

4.3. "Interior" semidiscrete systems. In order to define the semidiscrete observation system for the generator $G_{h}$ :

$$
\dot{v}^{h}(t)-i G_{h} v^{h}(t)=0, \quad v^{h}(0)=v_{0}^{h} \in H^{h}, \quad y^{h}(t)=C_{h} v^{h}(t),
$$

it seems natural to approximate the observation operator $C$ by

$$
C_{h}=C J_{h}, \quad C \in \mathscr{L}\left(H_{1} ; Y\right) .
$$

In order for this definition of $C_{h}$ to make sense we must assume $C \in \mathscr{L}\left(H_{1} ; Y\right)$. As already mentioned in $\$ 3.2 .3$, this assumption is in-between the general case $C \in$ $\mathscr{L}\left(\mathrm{H}_{2} ; \mathrm{Y}\right)$ adapted to boundary observability of PDEs and the bounded case $C \in$ $\mathscr{L}\left(H_{0} ; Y\right)$ adapted to interior observability of PDEs.

The norm of $C_{h}$ in $\mathscr{L}\left(V_{1}^{h} ; Y\right)$ is the norm of $C$ in $\mathscr{L}\left(H_{1} ; Y\right)$.

4.3.1. Convergence of the observation systems. In this framework, the following proposition discusses the convergence of the discrete observation $y^{h}$ in (74) to the continuous observation $y$ in (28) depending on the convergence of the initial data $J^{h} v_{0}^{h}$ to $x_{0}$ in $H_{1}$. It uses the following lemma.

Lemma 4.9. Assume (28) is admissible. Consider $\left(v^{h}\right)_{h>0}$ in $V^{h}$ and $x \in H_{0}$. Weakly in $L^{2}(0, T ; Y): C_{h} e^{i t G_{h}} G_{h}^{-1} v^{h} \rightarrow C e^{i t A} A^{-1} x$ implies $C_{h} e^{i t G_{h}} v^{h} \longrightarrow$ $C e^{i t A} x$.

Proof. If $x \in \mathscr{D}(A)$ then $C A^{-1} e^{i t A} x \in C^{1}(0, T ; Y)$ hence the following integration by parts is justified, therefore by admissibility of (28) and density of $\mathscr{D}(A)$ in $H_{0}$ :

$$
i \int_{0}^{T}\left\langle C A^{-1} e^{i t A} x, \varphi^{\prime}(t)\right\rangle d t=\int_{0}^{T}\left\langle C e^{i t A} x, \varphi(t)\right\rangle d t
$$


for $x \in H_{0}$, and $\varphi \in C_{\text {comp }}^{1}(0, T ; Y)$. Since $H_{h}$ is finite dimensional, (74) is admissible and similarly

$$
i \int_{0}^{T}\left\langle C_{h} G_{h}^{-1} e^{i t G_{h}} v^{h}, \varphi^{\prime}(t)\right\rangle d t=\int_{0}^{T}\left\langle C_{h} e^{i t G_{h}} v^{h}, \varphi(t)\right\rangle d t, \quad v^{h} \in H^{h} .
$$

The density of $C_{\text {comp }}^{1}(0, T ; Y)$ in $L^{2}(0, T ; Y)$ completes the proof.

Proposition 4.10. Assume (75). Consider a family $\left(v^{h}\right)_{h>0}$ in $V^{h}$. If $J_{h} v^{h} \rightarrow x$ in $H_{1}$ then $C_{h} e^{i t G_{h}} v^{h} \rightarrow C e^{i t A} x$ in $Y$ uniformly on finite intervals. More precisely, (62) implies the convergence rate

$$
\left\|\left(C_{h} e^{i t G_{h}} J_{h}^{*}-C e^{i t A}\right) x\right\| \leqslant\|C\|_{\mathscr{L}\left(H_{1} ; Y\right)} c_{0} h\left(t\|x\|_{4}+2\|x\|_{2}\right),
$$

for all $x \in H_{4}, h>0, t \in \mathbb{R}$.

If $J_{h} v^{h} \rightarrow x$ in $H_{1}$ then $C_{h} e^{i t G_{h}} v^{h} \rightarrow C e^{i t A} x$ in $L^{2}(0, T ; Y)$ for all $T>0$.

If $J_{h} v^{h} \rightarrow x$ in $H_{-1}, x \in H_{0}$ and (28) is admissible then $C_{h} e^{i t G_{h}} v^{h} \rightarrow C e^{i t A} x$ in $L^{2}(0, T ; Y)$ for all $T>0$.

Proof. The first implication results from the convergence in $H_{1}$ in the first part of Theorem 4.7, since $C_{h} e^{i t G_{h}}=C e^{i t A_{h}} J_{h}$ and $C \in \mathscr{L}\left(H_{1} ; Y\right)$. Moreover (69) implies the convergence rate since $C_{h} e^{i t G_{h}} J_{h}^{*}-C e^{i t A}=C\left(e^{i t A_{h}}-e^{i t A}\right)$.

The strong convergence implication in the first part of Theorem 4.7 also implies the weak convergence implication: if $x^{h} \rightarrow x$ in $H_{1}$ then $e^{i t A_{h}} x^{h} \rightarrow e^{i t A} x$ in $H_{1}$ uniformly on finite intervals of $t$. Using $C_{h} e^{i t G_{h}}=C e^{i t A_{h}} J_{h}$ and $C \in \mathscr{L}\left(H_{1} ; Y\right)$ again results in the second implication in Proposition 4.10.

To prove the third implication, we assume $J_{h} v^{h}-x$ in $H_{-1}$, equivalently $J_{h} G_{h}^{-1} v^{h} \rightarrow A^{-1} x$ in $H_{1}$. By the second implication, we have $C_{h} e^{i t G_{h}} G_{h}^{-1} v^{h} \rightarrow$ $C A^{-1} e^{i t A} x$ in $L^{2}(0, T ; Y)$. Lemma 4.9 completes the proof.

4.3.2. From continuous to filtered discrete observability. We consider improved resolvent conditions for (28), $s \geqslant 0: \exists L_{s}, l_{s}, M_{s}, m_{s}>0$,

$$
\begin{gathered}
\|C x\|^{2} \leqslant \frac{L_{s}}{\lambda^{s}}\|(A-\lambda) x\|_{0}^{2}+l_{s}\|x\|_{0}^{2}, \quad x \in H_{0}, \lambda \geqslant \inf A, \\
\|x\|_{0}^{2} \leqslant \frac{M_{s}}{\lambda^{s}}\|(A-\lambda) x\|_{0}^{2}+m_{s}\|C x\|^{2}, \quad x \in H_{0}, \lambda \geqslant \inf A .
\end{gathered}
$$

Unfortunately such conditions do not imply the corresponding conditions for the semidiscrete system (74) uniformly with respect to $h$. Therefore we consider uniform conditions for the semidiscrete system (74) restricted to the filtered space $\mathbf{1}_{G_{h}<\eta / h^{\sigma}} V^{h}$, where $\eta$ and $\sigma$ are positive filtering parameters: $\exists L_{s}^{\prime}, l_{s}^{\prime}, M_{s}^{\prime}, m_{s}^{\prime}>0$,

$$
\begin{gathered}
\left\|C_{h} v\right\|^{2} \leqslant \frac{L_{s}^{\prime}}{\lambda^{s}}\left\|\left(G_{h}-\lambda\right) v\right\|_{0}^{2}+l_{s}^{\prime}\|v\|_{0}^{2}, \\
\|v\|_{0}^{2} \leqslant \frac{M_{s}^{\prime}}{\lambda^{s}}\left\|\left(G_{h}-\lambda\right) v\right\|_{0}^{2}+m_{s}^{\prime}\left\|C_{h} v\right\|^{2},
\end{gathered}
$$


where, in both equations, $v \in \mathbf{1}_{G_{h}<\eta / h^{\sigma}} V^{h}$ and $\eta / h^{\sigma} \geqslant \lambda \geqslant \inf A$.

Theorem 4.11. Assume (75). Recall the approximation assumption (62) with $c_{0}$.

If the continuous system (28) is admissible (resp. moreover exactly observable) then, for all $\eta>0$ (resp. for $\eta>0$ small enough), there exists $T>0$ such that the semidiscrete system (74) restricted to the filtered space $\mathbf{1}_{G_{h}<\eta / h} V^{h}$ is admissible in time $T$ (resp. moreover exactly observable in time $T$ ) uniformly in $h \in(0, \eta)$.

If the second order system (56) is admissible and exactly observable then, for all $\eta>0$ small enough, for all $T>0$, the semidiscrete system (74) restricted to the filtered space $\mathbf{1}_{G_{h}<\eta / h} V^{h}$ is admissible and exactly observable in time $T$ uniformly in $h \in(0, \eta)$.

If $C \in \mathscr{L}\left(H_{0} ; Y\right)$ and the second order system (56) is admissible and exactly observable then, for all $\eta>0$ small enough, for all $T>0$, the semidiscrete system (74) restricted to the filtered space $\mathbf{1}_{G_{h}<\eta / h^{4 / 3}} V^{h}$ is admissible and exactly observable in time $T$ uniformly in $h \in(0, \eta)$.

More precisely, for $\sigma=1$ and any $s \geqslant 0$, setting $c_{\eta}=\max \left\{\left(c_{0} \eta\right)^{2}, c_{0} \eta\right\}:$ for all $\eta>0$, (76) implies (78) with $L_{s}^{\prime}=4 L_{s}$ and $l_{s}^{\prime}=4 l_{s}+2\left(\|C\|^{2}+2 l_{s}+\right.$ $\left.2 L_{s} / \inf A^{s}\right) c_{\eta}^{2}$; for $\eta>0$ small enough such that $d_{s}=1-c_{\eta}^{2}\left(1+4 m_{s}\|C\|^{2}-\right.$ $\left.4 M_{s} / \inf A^{s}\right)>0$, (77) implies (79) with $M_{s}^{\prime}=2 M_{s} / d_{s}$ and $m_{s}^{\prime}=2 m_{s} / d_{s}$. Here $\|C\|=\|C\| \mathscr{L}\left(H_{1} ; Y\right)$.

Proof. According to Proposition 2.9, $\lambda \geqslant \inf A$ in (76), (77), (78), (79) can be equivalently replaced by $\lambda>0$ since inf $G_{h} \geqslant \inf A>0$. For $s=0$, the range of $\lambda$ can be equivalently replaced by $\lambda \in \mathbb{R}$. Thus, the first (resp. second) implication of the theorem results from the last part of the theorem with $s=0$ (resp. $s=1$ ) according to Theorems 2.3 and 2.4 (resp. Theorem 3.13 and Remark 3.17).

To prove the last part of the theorem let $v \in V^{h}$ and consider $x^{h}=A^{-1} J_{h} G_{h} v$. It satisfies $A x^{h}=J_{h} G_{h} v$ and $\pi_{h} x^{h}=J_{h} v$ due to (67), hence $x^{h}-J_{h} v=\left(I-\pi_{h}\right) x^{h}$,

$$
(A-\lambda) x^{h}-J_{h}\left(G_{h}-\lambda\right) v=-\lambda\left(I-\pi_{h}\right) x^{h}, \quad C x^{h}-C_{h} v=C\left(I-\pi_{h}\right) x^{h} .
$$

Using Lemma 4.4, $C \in \mathscr{L}\left(H_{1} ; Y\right)$ and (62), this implies $\left\|x^{h}-J_{h} v\right\|_{0} \leqslant c_{0}^{2} h^{2}\left\|A x^{h}\right\|_{0}$,

$$
\begin{gathered}
\left\|(A-\lambda) x^{h}-J_{h}\left(G_{h}-\lambda\right) v\right\|_{0} \leqslant c_{0}^{2} h^{2} \lambda\left\|A x^{h}\right\|_{0}, \\
\left\|C x^{h}-C_{h} v\right\| /\|C\| \leqslant c_{0} h\left\|A x^{h}\right\|_{0} .
\end{gathered}
$$

Let $\sigma=1, s>0, \eta>0$ and $\eta / h \geqslant \lambda>0$. For $v \in \mathbf{1}_{G_{h}<\eta / h} V^{h},\left\|A x^{h}\right\|_{0}=$ $\left\|G_{h} v\right\|_{0} \leqslant(\eta / h)\|v\|_{0}$. Hence all the above norms are bounded by $c_{\eta}\|v\|_{0}$ for $h<\eta$. Plugging these bounds in (76) and (77) for $x^{h}$, and factoring out $\|v\|_{0}^{2}$ yields (78) and (79) with the constants stated in the theorem.

To prove the third implication of the theorem, we assume $C \in \mathscr{L}\left(H_{0} ; Y\right)$, (76), and (77) with $s=1$. The above bound is now replaced by

$$
\left\|C x^{h}-C_{h} v\right\| /\|C\| \leqslant c_{0}^{2} h^{2}\left\|A x^{h}\right\|_{0},
$$


which is better than $\lambda^{-s / 2}\left\|(A-\lambda) x^{h}-J_{h}\left(G_{h}-\lambda\right) v\right\|_{0} \leqslant c_{0}^{2} h^{2} \lambda^{1-s / 2}\left\|A x^{h}\right\|_{0}$ for $s<2, \lambda>1$. For $v \in \mathbf{1}_{G_{h}<\eta / h^{4 / 3}} V^{h},\left\|A x^{h}\right\|_{0}=\left\|G_{h} v\right\|_{0} \leqslant(\eta / h)^{4 / 3}\|v\|_{0}$. Plugging these bounds in (76) and (77) with $s=1$ for $x^{h}$, yield (78) and (79) with $s=1$ and errors bounded by the square of $c_{0}^{2} h^{2}\left((\eta / h)^{4 / 3}\right)^{1-1 / 2+1}\|v\|_{0}$ for $h<\eta$, where the power of $h$ is zero.

4.3.3. From filtered discrete to continuous observability. We prove that generalized resolvent conditions for the continuous system (28),

$$
\begin{gathered}
\|C x\|^{2} \leqslant L(\lambda)\|(A-\lambda) x\|_{0}^{2}+l(\lambda)\|x\|_{0}^{2}, \quad x \in \mathscr{D}(A), \lambda>\lambda_{0} \geqslant 0, \\
\|x\|_{0}^{2} \leqslant M(\lambda)\|(A-\lambda) x\|_{0}^{2}+m(\lambda)\|C x\|^{2}, \quad x \in \mathscr{D}(A), \lambda>\lambda_{0} \geqslant 0,
\end{gathered}
$$

where $l, L, m$ and $M$ are bounded positive functions on $(0,+\infty)$, can be obtained as the $h$-limit of the following $h$-uniform resolvent conditions for the semidiscrete system (74) restricted to the filtered space $\mathbf{1}_{G_{h}<f(h)} V^{h}$, where $f:\left(0, h_{0}\right) \rightarrow(0,+\infty)$ decreases and $f(h) \rightarrow+\infty$ as $h \rightarrow 0$ :

$$
\begin{gathered}
\left\|C_{h} v\right\|^{2} \leqslant L(\lambda)\left\|\left(G_{h}-\lambda\right) v\right\|_{0}^{2}+l(\lambda)\|v\|_{0}^{2}, \\
\|v\|_{0}^{2} \leqslant M(\lambda)\left\|\left(G_{h}-\lambda\right) v\right\|_{0}^{2}+m(\lambda)\left\|C_{h} v\right\|^{2},
\end{gathered}
$$

where, in both equations, $v \in \mathbf{1}_{G_{h}<f(h)} V^{h}$, and $\lambda \in\left[\lambda_{0}, f(h)\right)$.

Theorem 4.12. Assume (75). The semidiscrete admissibility (resp. observability) resolvent condition (82) implies (80) (resp. (83) implies (81)).

Proof. Consider $x \in \mathscr{D}(A)$ and $\mu>\lambda>\lambda_{0}$. It is enough to prove (80) and (81) for $x$ replaced by $\mathbf{1}_{A<\mu} x$ since $\left\|x-\mathbf{1}_{A<\mu} x\right\|_{2}=\left\|A \mathbf{1}_{A \geqslant \mu} x\right\|_{0} \rightarrow 0$ as $\mu \rightarrow \infty$. Let $h_{\mu}>0$ such that $f\left(h_{\mu}\right)>\mu$, and let $v^{h}=\mathbf{1}_{G_{h}<\mu} J_{h}^{*} \pi_{h} x \in \mathbf{1}_{G_{h}<f(h)} V^{h}$ for all $h \in$ $\left(0, h_{\mu}\right)$. Since $\mathbf{1}_{A_{h}<\mu} \pi_{h}-\mathbf{1}_{A<\mu}=\left(\mathbf{1}_{A_{h}<\mu}-\mathbf{1}_{A<\mu}\right) \pi_{h}+\mathbf{1}_{A_{h}<\mu}\left(I-\pi_{h}\right)$, using (68) and (62) yields $\left\|J_{h} v^{h}-\mathbf{1}_{A<\mu} x\right\|_{1}=\left\|\left(\mathbf{1}_{A_{h}<\mu} \pi_{h}-\mathbf{1}_{A<\mu}\right) x\right\|_{1} \rightarrow 0$ as $h \rightarrow 0$. Since $C \in \mathscr{L}\left(H_{1} ; Y\right)$, this convergence implies $\left\|C_{h} v^{h}-C \mathbf{1}_{A<\mu} x\right\|=\| C\left(J_{h} v^{h}-\right.$ $\left.\mathbf{1}_{A<\mu}\right) x \| \rightarrow 0$. Since $J_{h} G_{h} v^{h}=J_{h} \mathbf{1}_{G_{h}<\mu} G_{h} J_{h}^{*} \pi_{h} A^{-1} A x=\mathbf{1}_{A_{h}<\mu} A x$ due to (67), since $\left\|\left(\mathbf{1}_{A<\mu}-\mathbf{1}_{A_{h}<\mu}\right) A x\right\|_{0} \rightarrow 0$ due to (68), the convergence $J_{h} v^{h} \rightarrow$ $\mathbf{1}_{A<\mu} x$ in $H_{1}$ hence in $H_{0}$ implies $\left\|J^{h}\left(G_{h}-\lambda\right) v^{h}-(A-\lambda) \mathbf{1}_{A<\mu} x\right\|_{0} \rightarrow 0$. Therefore, taking the limit $h \rightarrow 0$ in (82) and (83) for $v^{h}$ implies (80) and (81) for $\mathbf{1}_{A<\mu} x$.

4.3.4. Convergence of the filtered control systems. We consider the semidiscrete control system dual to (74) with $B_{h}=\left(A^{1 / 2} J_{h}\right)^{*} A^{-1 / 2} B=G_{h} J_{h}^{*} \pi_{h} A^{-1} B$ (which is both the dual of $C_{h}: V_{1}^{h} \rightarrow Y$ and the adjoint of $C_{h}: V_{0}^{h} \rightarrow Y$ ),

$$
\dot{\psi}^{h}(t)-i G_{h} \psi^{h}(t)=B_{h} u^{h}, \quad \psi^{h}(0)=\psi_{0}^{h} \in H^{h}, \quad u^{h} \in L_{\mathrm{loc}}^{2}(\mathbb{R} ; Y) .
$$


The norm of $B_{h}$ in $\mathscr{L}\left(Y ; V_{-1}^{h}\right)$ is the norm of $C$ in $\mathscr{L}\left(H_{1} ; Y\right)$. According to Theorems 2.3 and 2.4 , the admissibility and exact observability resolvent conditions (82) and (83) for constant $l, L, m$ and $M$ are equivalent to the admissibility and exact observability of (74) restricted to the filtered space $\mathbf{1}_{G_{h}<f(h)} V^{h}$ for some $T>0$ uniformly in $h$. As in definitions 2.1 and 2.2, the dual notions for (84) are the following. There is an admissibility cost $K_{T}$ such that:

$$
\left\|\int_{0}^{T} e^{i t G_{h}} B_{h} u^{h}(t) d t\right\|^{2} \leqslant K_{T} \int_{0}^{T}\left\|u^{h}(t)\right\|^{2} d t, \quad u^{h} \in L^{2}(\mathbb{R} ; Y) .
$$

There is a controllability cost $\kappa_{T}>0$ such that: $\forall \psi_{0} \in H^{h}, \exists u^{h} \in L^{2}(\mathbb{R} ; Y)$,

$$
\begin{gathered}
u^{h}(t)=0, \quad t \notin[0, T], \\
\mathbf{1}_{G_{h}<f(h)} \psi^{h}(T)=0, \quad \text { and } \\
\int_{0}^{T}\left\|u^{h}(t)\right\|^{2} d t \leqslant \kappa_{T}\left\|\psi_{0}^{h}\right\|_{0}^{2} .
\end{gathered}
$$

The following theorem discusses the convergence of the inputs $u^{h}$ for the discrete system (84) to the input $u$ for the continuous system (29) depending on the convergence of the initial data $J_{h} \psi_{0}^{h}$ to $\xi_{0}$. It needs the notion of minimal control investigated by Jacques-Louis Lions, a.k.a. HUM control after the Hilbert Uniqueness Method he introduced (cf. [25]), and the characterization of this minimal control $u$ in (29) as the only control being also an observation $y$ in (28). For completeness, we prove this result and the same result for the discrete system (84).

Proposition 4.13. If (28) is admissible and exactly observable in time $T$ at cost $\kappa_{T}$ then among all the inputs $u$ such that the solution of (29) satisfies $\xi(T)=0$, there is one of minimal norm in $L^{2}(0, T ; Y)$ and this is the only one for which there exists $x_{0} \in H_{0}$ such that $u(t)=C e^{i t A} x_{0}, t \in[0, T]$. Moreover it satisfies

$$
\left\|x_{0}\right\|_{0} \leqslant \kappa_{T}\left\|\xi_{0}\right\|_{0} \quad \text { and } \quad-\left\langle x_{0}, \xi_{0}\right\rangle_{0}=\int_{0}^{T}\|u(t)\|^{2} d t \leqslant \kappa_{T}\left\|\xi_{0}\right\|_{0}^{2} .
$$

If (74) restricted to the filtered space $\mathbf{1}_{G_{h}<f(h)} V^{h}$ is exactly observable in time $T$, among all the inputs $u^{h}$ such that the solution of (84) satisfies $\mathbf{1}_{G_{h}<f(h)} \psi^{h}(T)=0$, there is one of minimal norm in $L^{2}(0, T ; Y)$ and this is the only one for which there exists $v_{0}^{h} \in \mathbf{1}_{G_{h}<f(h)} V^{h}$ such that $u^{h}(t)=C_{h} e^{i t G_{h}} v_{0}^{h}, t \in[0, T]$.

Proof. The Hilbert space $H_{0}$ with the hermitian scalar product $\langle\cdot, \cdot\rangle_{0}$ is also a real Hilbert space with the scalar product $\Re\langle\cdot, \cdot\rangle_{0}$. Consider the strictly convex $\ell^{1}$ functional $J$ defined on the real Hilbert space $H_{0}$ by density and admissibility as

$$
J(x)=\frac{1}{2} \int_{0}^{T}\left\|C e^{i t A} x\right\|^{2} d t+\Re\left\langle x, \xi_{0}\right\rangle_{0}, \quad x \in \mathscr{D}(A) .
$$


Exact observability implies $J(x) \geqslant \frac{1}{2 \kappa_{T}}\|x\|^{2}+\Re\left\langle x, \xi_{0}\right\rangle_{0}$, hence $J$ is coercive. Therefore $J$ has a unique minimizer $x_{*} \in H_{0}$, i.e. $J\left(x_{*}\right)=\inf _{x \in H_{0}} J(x)$, and the gradient $\nabla J$ computed with respect to the real scalar product $\Re\langle\cdot, \cdot\rangle_{0}$ vanishes at $x_{*}$, hence

$$
0=e^{i T A} \nabla J\left(x_{*}\right)=\int_{0}^{T} e^{i(T-t) A} B C e^{i t A} x_{*} d t+e^{i T A} \xi_{0} .
$$

This equation also says that the solution of (29) with the input $u_{*}(t)=C e^{i t A_{1}} x_{*}$ reaches the final state $\xi(T)=0$. In terms of this $u_{*},\left\langle\nabla J\left(x_{*}\right), x_{*}\right\rangle_{0}=0$ writes

$$
\int_{0}^{T}\left\|u_{*}(t)\right\|^{2} d t=\int_{0}^{T}\left\|C e^{i t A} x_{*}\right\|^{2} d t=-\left\langle x_{*}, \xi_{0}\right\rangle_{0} .
$$

The second equality and observability yield $\frac{1}{\kappa_{T}}\left\|x_{*}\right\|_{0}^{2} \leqslant-\left\langle x_{*}, \xi_{0}\right\rangle_{0} \leqslant\left\|x_{*}\right\|_{0}\left\|\xi_{0}\right\|_{0}$. Hence $\left\|x_{*}\right\|_{0} \leqslant \kappa_{T}\left\|\xi_{0}\right\|_{0}$. Plugging this in (89) yields (87) for $x_{*}$ and $u_{*}$.

Integrating by parts in time the scalar product in $H_{0}$ of (28) and (29) yields:

$$
\left\langle e^{i T A} x_{0}, \xi(T)\right\rangle_{0}=\left\langle x_{0}, \xi_{0}\right\rangle_{0}+\int_{0}^{T}\left\langle C e^{i t A} x_{0}, u(t)\right\rangle d t,
$$

for all $x_{0}, \xi_{0}$ in $H_{0}$ and $u$ in $L^{2}(\mathbb{R} ; Y)$. Thus $u$ controls $\xi_{0}$ in time $T$ if and only if

$$
0=\left\langle x, \xi_{0}\right\rangle_{0}+\int_{0}^{T}\left\langle C e^{i t A} x, u(t)\right\rangle d t, \quad x \in H_{0} .
$$

The minimality of $u_{*}$ results from this consequence of (89) and (91) with $x=x_{*}$ :

$$
\begin{aligned}
\left\|u_{*}\right\|_{L^{2}(0, T ; Y)}^{2} & =\int_{0}^{T}\left\|u_{*}(t)\right\|^{2} d t \\
& =\int_{0}^{T}\left\langle u_{*}(t), u(t)\right\rangle d t \\
& \leqslant\left\|u_{*}\right\|_{L^{2}(0, T ; Y)}\|u\|_{L^{2}(0, T ; Y)} .
\end{aligned}
$$

Writing (91) twice with $x=x_{0}-x_{*}$, once with $u(t)=C e^{i t A} x_{0}$ and once with $u_{*}(t)=C e^{i t A} x_{*}$, then taking the difference of the two equations yields

$$
0=\int_{0}^{T}\left\langle C e^{i t A} x, u(t)-u_{*}(t)\right\rangle d t=\int_{0}^{T}\left\|C e^{i t A} x\right\|^{2} d t .
$$

Exact observability deduces $\|x\|^{2}=0$, therefore $x_{0}=x_{*}$ hence $u=u_{*}$.

The second implication is proved similarly considering the functional $J_{h}$ defined on the real Hilbert space $\mathbf{1}_{G_{h}<f(h)} V^{h}$ with scalar product $\mathfrak{R}\langle\cdot, \cdot\rangle_{0}$ by

$$
J_{h}(v)=\frac{1}{2} \int_{0}^{T}\left\|C_{h} e^{i t G_{h}} v\right\|^{2} d t+\Re\left\langle v, \psi_{0}\right\rangle_{0}, \quad v \in \mathbf{1}_{G_{h}<f(h)} V^{h} .
$$


The projection $\mathbf{1}_{G_{h}<f(h)}$ commutes with $e^{i T G_{h}}$ and, for this scalar product,

$$
\nabla J_{h}(v)=\mathbf{1}_{G_{h}<f(h)} \int_{0}^{T} e^{-i t G_{h}} B_{h} C_{h} e^{i t G_{h}} v d t+\mathbf{1}_{G_{h}<f(h)} \psi_{0} .
$$

The solution of (84) with $u^{h}$ satisfies $\mathbf{1}_{G_{h}<f(h)} \psi^{h}(T)=0$ if and only if, as (91),

$$
0=\left\langle v_{0}^{h}, \psi_{0}^{h}\right\rangle_{0}+\int_{0}^{T}\left\langle C_{h} e^{i t G_{h}} v_{0}^{h}, u^{h}(t)\right\rangle d t, \quad v_{0}^{h} \in \mathbf{1}_{G_{h}<f(h)} V^{h} .
$$

Admissibility holds since $\mathbf{1}_{G_{h}<f(h)} V^{h}$ is finite dimensional (this proposition does not mention uniformity in $h$ ).

Theorem 4.14. Assume (75). Consider a family of initial data $\left(\psi_{0}^{h}\right)_{h>0}$ in $V^{h}$ and a family of inputs $\left(u^{h}\right)_{h>0}$ in $L^{2}(0, T ; Y)$ such that $u^{h}$ controls $\psi_{0}^{h}$ in time $T$ in the filtered space $\mathbf{1}_{G_{h}<f(h)} V^{h}$, i.e. the solution of (84) satisfies $\mathbf{1}_{G_{h}<f(h)} \psi^{h}(T)=0$.

If $J_{h} \psi_{0}^{h} \rightarrow \xi_{0}$ weakly in $H_{-1}$ and $u^{h} \rightarrow u$ weakly in $L^{2}(0, T ; Y)$ then u controls $\xi_{0}$ in time $T$, i.e. the solution of (97) satisfies $\xi(T)=0$.

Assume the discrete system (74) restricted to the filtered space $\mathbf{1}_{G_{h}<f(h)} V^{h}$ with the $V_{0}^{h}$ norm and the system (28) are admissible and exactly observable in time $T$ uniformly in $h$ (equivalently (85) and (86) hold, and (29) is admissible and exactly controllable in time $T$ ). If $J_{h} \psi_{0}^{h} \rightarrow \xi_{0}$ in $H_{0}$ then the minimal control $u^{h}$ of $\psi_{0}^{h}$ converges in $L^{2}(0, T ; Y)$ to the minimal control $u$ of $\xi_{0}$ given in Proposition 4.13. E.g. this applies to $\psi_{0}^{h}=J_{h}^{*} \xi_{0}$ for all $\xi_{0}$ in $H_{0}$, cf. Lemma 4.8 .

Proof. As in the proof of Proposition 4.13, integrating by parts in time the duality pairing between $H_{1}$ and $H_{-1}$ of (96) and (97), $u$ controls $\xi_{0}$ if and only if

$$
0=\left\langle x_{0}, \xi_{0}\right\rangle_{1,-1}+\int_{0}^{T}\left\langle C e^{i t A} x_{0}, u(t)\right\rangle d t, \quad x_{0} \in H_{1},
$$

and $u^{h}$ controls $\psi_{0}^{h}$ in time $T$ in the filtered space $\mathbf{1}_{G_{h}<f(h)} V^{h}$ if and only if (92). As in the proof of Theorem 4.12, consider $x_{0} \in \mathcal{D}(A), \mu>\lambda>0, h_{\mu}>0$ such that $f\left(h_{\mu}\right)>\mu$, and $v_{0}^{h}=\mathbf{1}_{G_{h}<\mu} J_{h}^{*} \pi_{h} x_{0} \in \mathbf{1}_{G_{h}<f(h)} V^{h}$ for all $h \in\left(0, h_{\mu}\right)$. Recall from the proof of Theorem 4.12, $J_{h} v_{0}^{h} \rightarrow \mathbf{1}_{A<\mu} x_{0}$ in $H_{1}$ as $h \rightarrow 0$. Proposition 4.10 deduces $C_{h} e^{i t G_{h}} v_{0}^{h} \rightarrow C e^{i t A} x_{0}$ in $L^{2}(0, T ; Y)$. Therefore the two assumptions of the first implication allows taking the limit $h \rightarrow 0$ in (92) and yield (93) for $x_{0}$ replaced by $\mathbf{1}_{A<\mu} x_{0}$. Taking the limit $\mu \rightarrow \infty$ and recalling from the proof of Theorem $4.12 \mathbf{1}_{A<\mu} x_{0} \rightarrow x_{0}$ in $H_{2}$, hence in $H_{1}$, yield (93) for $x_{0}$ in $H_{2}$, hence in $H_{1}$ by density. This completes the proof of the first implication.

Let $u^{h}(t)=C_{h} e^{i t G_{h}} v_{0}^{h}$ be the minimal control of $\psi_{0}^{h}$ and $u_{*}(t)=C e^{i t A} x_{*}$ be the minimal control of $\xi_{0}$ provided by the Hilbert Uniqueness Method in Proposition 4.13. Similarly to (87) we have

$$
\left\|v_{0}^{h}\right\|_{0} \leqslant \kappa_{T}\left\|\psi_{0}^{h}\right\|_{0} \quad \text { and } \quad-\left\langle v_{0}^{h}, \psi_{0}^{h}\right\rangle_{0}=\int_{0}^{T}\left\|u^{h}(t)\right\|^{2} d t \leqslant \kappa_{T}\left\|\psi_{0}^{h}\right\|_{0}^{2},
$$


where $\kappa_{T}$ is the uniform controllability cost in (86). Since $\psi_{0}^{h}$ converges in $H_{0}$, we deduce that $J^{h} v_{0}^{h}$ and $u^{h}$ are bounded respectively in $H_{0}$ and $L^{2}(0, T ; Y)$. We deduce that, after extracting a sequence if needed, $\left(u^{h}\right)$ has a weak limit $u$ in $L^{2}(0, T ; Y)$ and, after extracting again a sequence if needed, $\left(J^{h} v_{0}^{h}\right)$ has a weak limit $x_{0}$ in $H_{0}$. Since $J_{h} \psi_{0}^{h} \rightarrow \xi_{0}$ in $H_{0}$ hence in $H_{-1}$, the first implication now ensures that $u$ controls $\xi_{0}$ in time $T$. Since $J^{h} v_{0}^{h} \rightarrow x_{0}$ in $H_{0}$ hence in $H_{-1}$, Proposition 4.10 ensures $u^{h}(t)=C_{h} e^{i t G_{h}} v_{0}^{h} \rightarrow C e^{i t A} x_{0}$ in $L^{2}(0, T ; Y)$. Therefore $u(t)=C e^{i t A} x_{0}$ in $L^{2}(0, T ; Y)$. Hence Proposition 4.13 ensures that $x_{0}=x_{*}$ and $u=u_{*}$. Thus $u_{*}$ is the only accumulation point of $\left(u^{h}\right)$ weakly in $L^{2}(0, T ; Y)$, hence $u^{h} \rightarrow u_{*}$ in $L^{2}(0, T ; Y)$. Since $J^{h} v_{0}^{h} \rightarrow x_{0}=x_{*}$ in $H_{0}$ and $J^{h} \psi_{0}^{h} \rightarrow \xi_{0}$ in $H_{0}$, the left hand side of the equality in (94) converges to the right hand side of (89). Hence the norm of $u^{h}$ converges to the norm of $u_{*}$. Since we already proved the weak convergence of $u^{h}$ to $u_{*}$ in $L^{2}(0, T ; Y)$, this proves the strong convergence, which completes the proof of the second implication.

Remark 4.15. The investigation in $\S 4$ was triggered by the approach introduced by Sylvain Ervedoza in [11] under the assumptions $C \in \mathscr{L}\left(H_{\gamma} ; Y\right), \gamma \in[0,1)$ and $A^{-1}$ is compact. After the version of [12] and [13] in [11] was submitted, the author privately communicated to Ervedoza an improvement of the filtering scale now included in [12] and [13]. E.g. with the current notations Ervedoza proved his main theorem with (6.1.11) in [11], $\sigma=\min \{2(1-\gamma), 2 / 5\}$, using a version of the resolvent condition which he called interpolation inequalities; the author improved it to $(1.11)$ in [13], $\sigma=\min \{2(1-\gamma), 2 / 3\}$, using Proposition 2.9 instead. Similarly, the author improved (7.1.12) in [11] into (1.12) in [12], cf. [12], Remark 3.1.

Theorem 4.11 improves Theorem 1.3 in [13] in four ways: the approximation assumption (62) is weaker in practice (cf. Remark 4.1), the assumption on the observation operator $C \in \mathscr{L}\left(H_{1} ; Y\right)$ is weaker, $A^{-1}$ is not assumed to be compact, the filtering power $\sigma=\min \{2(1-\gamma), 2 / 3\}$ is improved into $\sigma=1$.

The converse Trotter-Kato type Theorem 4.12 is not considered in [13].

The second part of Theorem 4.14 improves Theorem 6.2 in [13] by eliminating, thanks to Lemma 4.9, the dubiously used assumption (6.11) in [13] which limited to $C \in \mathscr{L}\left(H_{0} ; Y\right)$ the validity of Theorem 6.2 in [13] and the validity of the convergence of the observation. Proposition 4.10 also improves this convergence of the observation in particular by providing explicit convergence rates. Theorem 4.7 is proved here, with no claim of originality, since we could not find the proof of the similar Lemma 6.4 in [13] in the reference given for it.

4.4. "Boundary" semidiscrete systems. To address the general case of $\S 3$ which suits boundary observability of PDEs, i.e. $\mathrm{C} \in \mathscr{L}\left(\mathrm{H}_{2} ; \mathrm{Y}\right)$, we need to modify the definition (75) of the approximate observation operator. Thus we consider the semidiscrete observation system (74) with (75) replaced by:

$$
C_{h}=C A^{-1} J_{h} G_{h}, \quad C \in \mathscr{L}\left(H_{2} ; Y\right) .
$$


Note that $\left\|C_{h} v\right\| \leqslant\|C\|_{\mathscr{L}\left(H_{2} ; Y\right)}\left\|G_{h} v\right\|_{0}$ but the $V_{2}^{h}$ norm $\|v\|_{2}$ was not defined as $\left\|G_{h} v\right\|_{0}$.

4.4.1. Convergence of the observation systems. Similarly to Proposition 4.10:

Proposition 4.16. Assume (95). Consider a family $\left(v^{h}\right)_{h>0}$ in $V^{h}$ and $x \in \mathscr{D}(A)$. If $J_{h} G_{h} v^{h} \rightarrow A x$ in $H_{0}$ then $C_{h} e^{i t G_{h}} v^{h} \rightarrow C e^{i t A} x$ in $Y$ uniformly on finite intervals of $t$. More precisely, (62) implies the convergence rate: $\forall h>0, \forall t \in \mathbb{R}$,

$$
\left\|\left(C_{h} e^{i t G_{h}} J_{h}^{*} \pi_{h}-C e^{i t A}\right) x\right\| \leqslant\|C\|_{\mathscr{L}\left(H_{2} ; Y\right)} c_{0}^{2} h^{2}\left(t\|x\|_{6}+2\|x\|_{4}\right), \quad x \in H_{6} .
$$

If $J_{h} G_{h} v^{h} \rightarrow$ Ax in $H_{0}$ then $C_{h} e^{i t G_{h}} v^{h} \rightarrow C e^{i t A} x$ in $L^{2}(0, T ; Y)$ for all $T>0$. If $J_{h} v^{h} \rightarrow x$ in $H_{0}, x \in H_{1}$ and (96) is admissible then $C_{h} e^{i t G_{h}} v^{h} \rightarrow C e^{i t A_{x}}$ in $L^{2}(0, T ; Y)$ for all $T>0$.

Proof. The first implication results from the convergence in $H_{0}$ in Theorem 4.7, since $C_{h} e^{i t G_{h}}=C A^{-1} e^{i t A_{h}} J_{h} G_{h}$ and $C A^{-1} \in \mathscr{L}\left(H_{0} ; Y\right)$. Since (67) is equivalent to $A=J_{h} G_{h} J_{h}^{*} \pi_{h}$ on $\mathcal{D}(A)$, we have $C_{h} e^{i t G_{h}} J_{h}^{*} \pi_{h}-C e^{i t A}=C A^{-1}\left(e^{i t A_{h}}-e^{i t A}\right) A$. Therefore (70) implies the convergence rate in Proposition 4.16.

The second implication in Proposition 4.16. is proved as in Proposition 4.10.

To prove the third implication, we rewrite the assumption $J_{h} v^{h} \rightarrow x$ in $H_{0}$, as $J_{h} G_{h}\left(G_{h}^{-1} v^{h}\right) \rightarrow A^{-1}(A x)$ in $H_{0}$. The second implication yields $C_{h} e^{i t G_{h}} G_{h}^{-1} v^{h} \rightarrow$ $C A^{-1} e^{i t A} x$ in $L^{2}(0, T ; Y)$. Since $x \in H_{1}$ and (96) is admissible, this completes the proof as in Lemma 4.9.

4.4.2. Continuous and filtered discrete observability. For boundary observation and in relation to the second order system (48), it is natural to consider the unitary group on $H_{1}$ instead of $H_{0}$, i.e. we consider (50) and (51) for $s=2$ :

$$
\begin{gathered}
\dot{x}(t)-i A x(t)=0, \quad x(0)=x_{0} \in H_{1}, \quad y(t)=C x(t), \\
\dot{\xi}(t)-i A^{\prime} \xi(t)=B u(t), \quad \xi(0)=\xi_{0} \in H_{-1}, \quad u \in L_{\mathrm{loc}}^{2}(\mathbb{R} ; Y) .
\end{gathered}
$$

We consider improved resolvent conditions for (96): $\exists L_{s}, l_{s}, M_{s}, m_{s}>0$,

$$
\begin{gathered}
\|C x\|^{2} \leqslant \frac{L_{s}}{\lambda^{s}}\|(A-\lambda) x\|_{1}^{2}+l_{s}\|x\|_{1}^{2}, \quad x \in H_{3}, \lambda>0, \\
\|x\|_{1}^{2} \leqslant \frac{M_{s}}{\lambda^{s}}\|(A-\lambda) x\|_{1}^{2}+m_{s}\|C x\|^{2}, \quad x \in H_{3}, \lambda>0 .
\end{gathered}
$$

Recall from (66) that the $V_{1}^{h}$ norm on $V^{h}$ is $\|v\|_{1}=\left\|J_{h} v\right\|_{1}=\left\|G_{h}^{1 / 2} v\right\|, v \in V^{h}$. We also consider the improved resolvent conditions (78) and (79) for the semidiscrete 
system (74) but with this $V_{1}^{h}$ norm instead of the $V_{0}^{h}$ norm: $\exists L_{s}^{\prime}, l_{s}^{\prime}, M_{s}^{\prime}, m_{s}^{\prime}>0$,

$$
\begin{gathered}
\left\|C_{h} v\right\|^{2} \leqslant \frac{L_{s}^{\prime}}{\lambda^{s}}\left\|\left(G_{h}-\lambda\right) v\right\|_{1}^{2}+l_{s}^{\prime}\|v\|_{1}^{2}, \\
\|v\|_{1}^{2} \leqslant \frac{M_{s}^{\prime}}{\lambda^{s}}\left\|\left(G_{h}-\lambda\right) v\right\|_{1}^{2}+m_{s}^{\prime}\left\|C_{h} v\right\|^{2},
\end{gathered}
$$

where, in both equations, $v \in \mathbf{1}_{G_{h}<\eta / h^{\sigma}} V^{h}$, and $\eta / h^{\sigma} \geqslant \lambda>0$. Similarly to Theorem 4.12, we have:

Theorem 4.17. Assume (95). The semidiscrete admissibility (resp. observability) resolvent condition (82) implies (80) (resp. (83) implies (81)) when replacing the $H_{0}$ norms by $H_{1}$ norms and $\mathscr{D}(A)$ by $H_{3}$ in all these resolvent conditions.

Proof. Few modifications of the proof of Theorem 4.12 are necessary.

Consider $x \in H_{3}$ and $\mu>\lambda \geqslant \lambda_{0}$. It is enough to prove (80) and (81) for $x$ replaced by $\mathbf{1}_{A<\mu} x$ since $\left\|x-\mathbf{1}_{A<\mu} x\right\|_{3}=\left\|A \mathbf{1}_{A \geqslant \mu} x\right\|_{1} \rightarrow 0$ as $\mu \rightarrow \infty$. Let $h_{\mu}>0$ such that $f\left(h_{\mu}\right)>\mu$, and let $v^{h}=\mathbf{1}_{G_{h}<\mu} J_{h}^{*} \pi_{h} x \in \mathbf{1}_{G_{h}<f(h)} V^{h}$ for all $h \in\left(0, h_{\mu}\right)$. Recall from the proof of Theorem 4.12 that $\left\|J_{h} v^{h} \rightarrow \mathbf{1}_{A<\mu} x\right\|_{1} \rightarrow 0$ as $h \rightarrow 0$ and $J_{h} G_{h} v^{h}=\mathbf{1}_{A_{h}<\mu} A x$. Moreover $\left\|\left(\mathbf{1}_{A<\mu}-\mathbf{1}_{A_{h}<\mu}\right) A x\right\|_{1} \rightarrow 0$ due to (68). Hence $\left\|J^{h}\left(G_{h}-\lambda\right) v^{h}-(A-\lambda) \mathbf{1}_{A<\mu} x\right\|_{1} \rightarrow 0$. Since $C_{h} v^{h}=C A^{-1} J_{h} G_{h} v^{h}=$ $C A^{-1} \mathbf{1}_{A_{h}<\mu} A x$ and $C A^{-1} \in \mathscr{L}\left(H_{0} ; Y\right)$ due to (95), this also implies $\| C_{h} v^{h}-$ $C \mathbf{1}_{A<\mu} x\|=\| C A^{-1}\left(\mathbf{1}_{A_{h}<\mu}-\mathbf{1}_{A<\mu}\right) A x \| \rightarrow 0$. Therefore, replacing the $H_{0}$ norms by $H_{1}$ norms and $\mathscr{D}(A)$ by $H_{3}$ and taking the limit $h \rightarrow 0$ in (82) and (83) for $v^{h}$ implies (80) and (81) for $\mathbf{1}_{A<\mu} x$.

Similarly to Theorem 4.11 for the direct implications and due to Theorem 4.12 for the converse implications:

Theorem 4.18. Assume that the observation operators satisfy (95).

The continuous system (96) is admissible (resp. moreover exactly observable) if and only if, for all $\eta>0$ (resp. for $\eta>0$ small enough), there exists $T>0$ such that the semidiscrete system (74) restricted to the filtered space $\mathbf{1}_{G_{h}<\eta / h^{2 / 3}} V^{h}$ with the $V_{1}^{h}$ norm is admissible (resp. moreover exactly observable) in time $T$ uniformly in $h \in(0,1)$.

If the second order system (48) is admissible and exactly observable then, for $\eta>0$ small enough, for all $T>0$, the semidiscrete system (74) restricted to the filtered space $\mathbf{1}_{G_{h}<\eta / h} V^{h}$ with the $V_{1}^{h}$ norm is admissible and exactly observable in time $T$ uniformly in $h \in(0,1)$.

The continuous system (28) is admissible (resp. moreover exactly observable) if and only if, for all $\eta>0$ (resp. for $\eta>0$ small enough), there exists $T>0$ such that the semidiscrete system (74) restricted to the filtered space $\mathbf{1}_{G_{h}<\eta / h} V^{h}$ with the $V_{0}^{h}$ norm is admissible (resp. moreover exactly observable) in time $T$ uniformly in $h \in(0,1)$. 
More precisely, for $s \in[0,4)$ and $\sigma=1 /(1-s / 4)$ (resp. $s \in[0,3)$ and $\sigma=$ $2 /(3-s)$ ), for all $\eta>0$, (76) implies (78) (resp. (98) implies (100)); for $\eta>0$ small enough (77) implies (79) (resp. (98) implies (100)).

Proof. According to Proposition 2.9, $\lambda>0$ in (98), (99), (100), (101) can be equivalently replaced by $\lambda \geqslant \inf A$ since inf $G_{h} \geqslant \inf A>0$. For $s=0$, the range of $\lambda$ can be equivalently replaced by $\lambda \in \mathbb{R}$. Thus, the first and third (resp. second) implication of the theorem results from the last part of the theorem with $s=0$ (resp. $s=1$ ) according to Theorems 2.3 and 2.4 (resp. Theorem 3.8).

To prove the last part of the theorem let $v \in V^{h}$ and consider $x^{h}=A^{-1} J_{h} G_{h} v$ as in the proof of Theorem 4.11. Due to (95), we now have $C_{h} v=C x^{h}$.

Recall from the proof of Theorem 4.11 that Lemma 4.4 implies:

$$
\left\|x^{h}-J_{h} v\right\|_{0} \leqslant c_{0}^{2} h^{2}\left\|A x^{h}\right\|_{0}, \quad\left\|(A-\lambda) x^{h}-J_{h}\left(G_{h}-\lambda\right) v\right\|_{0} \leqslant c_{0}^{2} h^{2} \lambda\left\|A x^{h}\right\|_{0} .
$$

For $v \in \mathbf{1}_{G_{h}<\eta / h^{\sigma}} V^{h},\left\|A x^{h}\right\|_{0}=\left\|G_{h} v\right\|_{0} \leqslant\left(\eta / h^{\sigma}\right)\|v\|_{0}$. Plugging these bounds in (76) and (77) for $x^{h}$ yield (78) and (79) with errors bounded by the square of $c_{\eta} h^{2-\sigma(2-s / 2)}\|v\|_{0}$ for $h<1$, where the power of $h$ is zero for $\sigma=1 /(1-s / 4)$, and $c_{\eta}=\max \left\{\left(c_{0} \eta\right)^{2}, c_{0} \eta\right\} \rightarrow 0$ as $\eta \rightarrow 0$.

Similarly, the approximation assumption (62) yields:

$$
\left\|x^{h}-J_{h} v\right\|_{1} \leqslant c_{0} h\left\|A x^{h}\right\|_{0}, \quad\left\|(A-\lambda) x^{h}-J_{h}\left(G_{h}-\lambda\right) v\right\|_{1} \leqslant c_{0} h \lambda\left\|A x^{h}\right\|_{0} .
$$

For $v \in \mathbf{1}_{G_{h}<\eta / h^{\sigma}} V^{h},\left\|A x^{h}\right\|_{0}=\left\|G_{h} v\right\|_{0} \leqslant\left(\eta / h^{\sigma}\right)^{1 / 2}\|v\|_{1}$. Plugging these bounds in (98) and (99) for $x^{h}$ yield (100) and (101) with errors bounded by the square of $c_{\eta} h^{1-\sigma(3-s) / 2}\|v\|_{0}$ for $h<1$, and the power of $h$ is now zero for $\sigma=2 /(3-s)$.

4.4.3. Convergence of the filtered control systems. We consider the semidiscrete control system (84) dual to (74) with $B_{h}=G_{h} J_{h}^{*} A^{-1} B$ (which is both the dual of $C_{h}: V_{2}^{h} \rightarrow Y$ and the adjoint of $\left.C_{h}: V_{0}^{h} \rightarrow Y\right)$. Note that $\left\|G_{h}^{-1} B_{h} y\right\|_{0} \leqslant$ $\|C\|_{\mathscr{L}\left(H_{2} ; Y\right)}\|y\|$.

Theorem 4.19. Assume (95). Consider a family of initial data $\left(\psi_{0}^{h}\right)_{h>0}$ in $V^{h}$ and a family of inputs $\left(u^{h}\right)_{h>0}$ in $L^{2}(0, T ; Y)$ such that $u^{h}$ controls $\psi_{0}^{h}$ in time $T$ in the filtered space $\mathbf{1}_{G_{h}<f(h)} V^{h}$, i.e. the solution of (84) satisfies $\mathbf{1}_{G_{h}<f(h)} \psi^{h}(T)=0$.

If $J_{h} \psi_{0}^{h} \rightarrow \xi_{0}$ weakly in $H_{-1}$ and $u^{h} \rightarrow u$ weakly in $L^{2}(0, T ; Y)$ then u controls $\xi_{0}$ in time $T$, i.e. the solution of (97) satisfies $\xi(T)=0$.

Assume the discrete system (74) restricted to the filtered space $\mathbf{1}_{G_{h}<f(h)} V^{h}$ with the $V_{1}^{h}$ norm and the system (96) are admissible and exactly observable in time $T$ uniformly in $h$. If $J_{h} \psi_{0}^{h} \rightarrow \xi_{0}$ in $H_{-1}$ then the minimal control $u^{h}$ of $\psi_{0}^{h}$ converges in $L^{2}(0, T ; Y)$ to the minimal control $u$ of $\xi_{0}$. E.g. this applies to $\psi_{0}^{h}=G_{h} J_{h}^{*} \pi_{h} A^{-1} \xi_{0}$ for all $\xi_{0}$ in $H_{-1}$, cf. Lemma 4.8 . 
Proof. Few modifications of the proof of Theorem 4.14 are necessary.

In the proof of the first implication, Proposition 4.16 replaces Proposition 4.10. In order to apply this Proposition 4.16 to $v_{0}^{h}$ as in the proof of Theorem 4.14, recall from the proof of Theorem 4.12 that $J_{h} G_{h} v_{0}^{h} \rightarrow A \mathbf{1}_{A<\mu} x_{0}$ in $H_{0}$ as $h \rightarrow 0$.

The proof of the second implication uses the Hilbert Uniqueness Method as in Proposition 4.13 but the $H_{0}$ scalar product in the functional (88) is replaced by the duality pairing between $H_{1}$ and $H_{-1}$. Thus (87) and (94) are replaced by

$$
\begin{gathered}
\left\|x_{0}\right\|_{1} \leqslant \kappa_{T}\left\|\xi_{0}\right\|_{-1} \quad \text { and } \quad-\left\langle x_{0}, \xi_{0}\right\rangle_{1,-1}=\int_{0}^{T}\|u(t)\|^{2} d t \leqslant \kappa_{T}\left\|\xi_{0}\right\|_{-1}^{2}, \\
\left\|v_{0}^{h}\right\|_{1} \leqslant \kappa_{T}\left\|\psi_{0}^{h}\right\|_{-1} \quad \text { and } \quad-\left\langle v_{0}^{h}, \psi_{0}^{h}\right\rangle_{1,-1}=\int_{0}^{T}\left\|u^{h}(t)\right\|^{2} d t \leqslant \kappa_{T}\left\|\psi_{0}^{h}\right\|_{-1}^{2} .
\end{gathered}
$$

Since $\psi_{0}^{h}$ converges in $H_{-1}$, we deduce that $J^{h} v_{0}^{h}$ and $u^{h}$ are bounded respectively in $H_{1}$ and $L^{2}(0, T ; Y)$. We deduce that, after extracting a sequence if needed, $\left(u^{h}\right)$ has a weak limit $u$ in $L^{2}(0, T ; Y)$ and, after extracting again a sequence if needed, $\left(J^{h} v_{0}^{h}\right)$ has a weak limit $x_{0}$ in $H_{1}$. Since $J_{h} \psi_{0}^{h} \rightarrow \xi_{0}$ in $H_{-1}$ strongly hence weakly, the first implication now ensures that $u$ controls $\xi_{0}$ in time $T$. Since $J^{h} v_{0}^{h} \rightarrow x_{0}$ in $H_{1}$ hence in $H_{0}$, Proposition 4.16 ensures $u^{h}(t)=C_{h} e^{i t G_{h}} v_{0}^{h} \rightarrow C e^{i t A_{x_{0}}}$ in $L^{2}(0, T ; Y)$. Therefore $u(t)=C e^{i t A} x_{0}$ in $L^{2}(0, T ; Y)$. As in Proposition 4.13, we deduce $x_{0}=x_{*}$ and $u=u_{*}$. Thus $u_{*}$ is the only accumulation point of $\left(u^{h}\right)$ weakly in $L^{2}(0, T ; Y)$, hence $u^{h} \rightarrow u_{*}$ in $L^{2}(0, T ; Y)$. Since $\left(J^{h} v_{0}^{h}\right) \rightarrow x_{0}=x_{*}$ in $H_{1}$ and $\left(J^{h} \psi_{0}^{h}\right) \rightarrow \xi_{0}$ in $H_{-1}$, the left hand side of the equality in (103) converges to the left hand side of the equality in (102). Hence the norm of $u^{h}$ converges to the norm of $u=u_{*}$. Since we already proved the weak convergence of $u^{h}$ to $u_{*}$ in $L^{2}(0, T ; Y)$, this proves the strong convergence, which completes the proof of the second implication.

\subsection{Second order semidiscrete systems}

4.5.1. Framework for the Galerkin approximation of second order systems. Section 3.2 explains how the second order observation systems both in the "boundary" case (48) and the "interior" case (56) fit in the general framework of $\S 2.1$. Since they are dual to each other, from now on the state space and the operator generating the observation system are denoted by $X$ and $\mathcal{A}$ in the "boundary" case, by $X^{\prime}$ and $\mathcal{A}^{\prime}$ in the "interior" case ( $X$ is the "energy space" for the wave equation). Using the new notation

$$
\begin{aligned}
Z_{s}=H_{s+1} \times H_{s}, \quad\left\|\left(z_{0}, z_{1}\right)\right\|_{s}^{2} & =\left\|A z_{0}\right\|^{2}+\left\|z_{1}\right\|^{2} \\
& =\left\|A^{(s+1) / 2} z_{0}\right\|_{0}^{2}+\left\|A^{s / 2} z_{1}\right\|_{0}^{2},
\end{aligned}
$$


these state spaces and operators are defined by

$$
\begin{array}{cc}
X=Z_{0}=X_{1}^{\prime}=\mathscr{D}\left(\mathcal{A}^{\prime}\right), & \mathscr{D}(\mathcal{A})=X_{1}=Z_{1}, \\
X_{-1}=Z_{-1}=X^{\prime}, & Z_{-2}=X_{-1}^{\prime},
\end{array}
$$

with duality pairing $\left\langle\left(z_{0}, z_{1}\right),\left(\zeta_{0}, \zeta_{1}\right)\right\rangle=\left\langle A^{1 / 2} z_{0}, A^{-1 / 2} \zeta_{1}\right\rangle_{0}-\left\langle z_{1}, \zeta_{0}\right\rangle_{0}$,

$$
\mathcal{A}\left(z_{0}, z_{1}\right)=i\left(-z_{1}, A z_{0}\right), \quad \mathcal{A}^{\prime}\left(z_{0}, z_{1}\right)=i\left(-z_{1}, A^{\prime} z_{0}\right) .
$$

Thus $\mathcal{A}^{\prime}$ is an extension of $\mathcal{A}$ to $Z_{-1}$ with domain $Z_{0}$.

The spectra and spectral projections of $\mathcal{A}$ and $A$ are linked by the isomorphism $W$ in the proof of Theorem (3.8):

$$
\sigma(\mathcal{A})=\sigma(\sqrt{A}) \cup \sigma(-\sqrt{A}), \quad \mathbf{1}_{\mathcal{A}^{2}<\lambda}\left(z_{0}, z_{1}\right)=\left(\mathbf{1}_{A<\lambda} z_{0}, \mathbf{1}_{A<\lambda} z_{1}\right) .
$$

In both cases the observation operator $C$ is bounded on the domain of the generator of the observation system, it is defined by $\varrho\left(z_{0}, z_{1}\right)=C z_{0}$ and the dual control operator $\mathscr{B}$ is defined by $\mathscr{B} y=(0, B y)$. Thus $\mathscr{C} \in \mathscr{L}\left(Z_{1} ; Y\right)$ and $\mathscr{B} \in \mathscr{L}\left(Y ; Z_{-2}\right)$ in the "boundary" case, $\mathcal{C} \in \mathscr{L}\left(Z_{0} ; Y\right)$ and $\mathscr{B} \in \mathscr{L}\left(Y ; Z_{-1}\right)$ in the "interior" case.

The approximation spaces are $W^{h}=W^{h} \times W^{h}$ with injections $\mathscr{g}_{h}: W^{h} \rightarrow Z_{0}$ defined by $g_{h}\left(w_{0}, w_{1}\right)=\left(J_{h} w_{0}, J_{h} w_{1}\right)$. Let $W_{s}^{h}$ denote $W^{h}$ with the norm $\|w\|_{s}=$ $\left\|\mathscr{A}_{h} w\right\|_{s}, w \in W^{h}$, induced by $\mathscr{g}_{h}$ from $Z_{s}$. The dual $\mathcal{G}_{h}^{*}: Z_{0} \rightarrow W_{0}^{h}$ is defined by $\mathcal{I}_{h}^{*}\left(z_{0}, z_{1}\right)=\left(J_{h}^{*} \pi_{h} z_{0}, J_{h}^{*} z_{1}\right)$. The $Z_{0}$-orthogonal projection from $Z_{0}$ onto $Z^{h}$ is

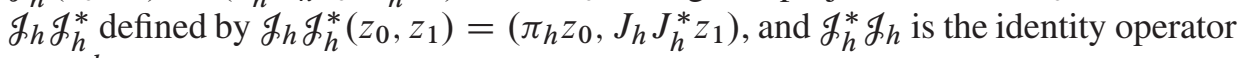
on $W^{h}$.

The Ritz-Galerkin variational method considers the finite-dimensional positive self-adjoint operator on $\mathscr{E}_{h}$ on $W_{0}^{h}$ defined by $\mathscr{G}_{h}\left(w_{0}, w_{1}\right)=i\left(-w_{1}, G_{h} w_{0}\right)$, approximates $\mathcal{A}$ by the non-negative selfadjoint operator $\mathcal{A}_{h}=\mathscr{A}_{h} \mathscr{E}_{h} \mathcal{F}_{h}^{*}$ on $Z_{0}$ defined by $\mathcal{A}_{h}\left(z_{0}, z_{1}\right)=\left(-J_{h}^{*} J_{h} z_{1}, A_{h} \pi_{h} z_{0}\right)$, approximates $C_{\text {by }}$ the observation operator $\bigodot_{h}$ defined by $\mathcal{C}_{h}\left(w_{0}, w_{1}\right)=C_{h} w_{0}$, and approximates $\mathcal{B}$ by the control operator $\mathcal{B}_{h}$ defined by $\mathscr{B}_{h} y=\left(0, B_{h} y\right)$. In the "interior" case the definition of $C_{h}$ is (75) thus $e_{h}=e_{\mathcal{G}_{h}}$, in the "boundary" case the definition of $C_{h}$ is (95) thus $\ell_{h}=e_{\mathcal{A}^{-1}} \mathscr{g}_{h} \varepsilon_{h}$.

The dual observation and control systems generated by $\mathscr{E}_{h}$ are:

$$
\begin{gathered}
\ddot{w}^{h}(t)+G_{h} w^{h}(t)=0, \quad w^{h}(0)=w_{0} \in V^{h}, \\
\dot{w}^{h}(0)=w_{1} \in V^{h}, \quad y(t)=C_{h} w^{h}(t), \\
\ddot{\Psi}^{h}(t)+G^{h} \Psi^{h}(t)=B_{h} u^{h}(t), \quad \Psi(0)=\Psi_{0} \in V^{h}, \\
\dot{\Psi}(0)=\Psi_{1} \in V^{h}, \quad u \in L_{\text {loc }}^{2}(\mathbb{R} ; Y) .
\end{gathered}
$$

We still denote by $\pi_{h}$ the projection defined on $H_{1} \times H_{1}$ by $\pi_{h}\left(z_{0}, z_{1}\right)=\left(\pi_{h} z_{0}, \pi_{h} z_{1}\right)$. The approximation assumption (62) and its consequence (63) write:

$$
\left\|z-\pi_{h} z\right\|_{0} \leqslant c_{0} h\|z\|_{1}, \quad z \in Z_{1}, h>0 .
$$


As in Lemma 4.6 , in terms of $\pi_{h}, \boldsymbol{E}_{h}$ is defined by

$$
\pi_{h} \mathcal{A}^{-1}=\mathscr{g}_{h} \mathscr{E}_{h}^{-1} \mathcal{g}_{h}^{*},
$$

hence $\left\|\left(\mathcal{A}^{-1}-\mathcal{G}_{h} \mathcal{E}_{h}^{-1} \mathcal{G}_{h}^{*}\right) z\right\|_{0} \leqslant c_{0} h\|z\|_{0}, x \in H_{0}$. Therefore $\mathcal{A}_{h}$ converges to $\mathcal{A}$ in $Z_{0}$ strongly in the resolvent sense. In particular, if $\lambda \in \mathbb{R}$ is not an eigenvalue of $\mathcal{A}$, then the finite dimensional spectral projections $\mathbf{1}_{\mathcal{A}_{h}<\lambda}=\mathscr{A}_{h} \mathbf{1}_{h}<\lambda \mathcal{G}_{h}^{*}$ satisfy

$$
\left\|\left(\mathbf{1}_{\mathcal{A}<\lambda}-\mathbf{1}_{\mathcal{A}_{h}<\lambda}\right) z\right\|_{0} \rightarrow 0, \quad z \in Z_{0} .
$$

The proof of Theorem 4.7, (107) and (108) yield:

Theorem 4.20. If $z^{h} \rightarrow z$ in $Z_{0}$ then $e^{i t \mathcal{A}_{h}} z^{h} \rightarrow e^{i t \mathcal{A}_{z}}$ in $Z_{0}$ uniformly on finite intervals of $t$, i.e. the solutions $z$ and $w$ of (48) and (105) satisfy

$$
\sup _{t \in[0, T]}\left(\left\|w^{h}(t)-z(t)\right\|_{1}+\left\|\dot{w}^{h}(t)-\dot{z}(t)\right\|_{0}\right) \rightarrow 0,
$$

with $(z(0), \dot{z}(0)) \in H_{1} \times H_{0}, T \in \mathbb{R}$. More precisely, (62) implies the convergence rate

$$
\left\|\left(e^{i t \mathscr{A}_{h}}-e^{i t \mathcal{A}}\right) z\right\|_{0} \leqslant c_{0} h\left(t\|z\|_{2}+2\|z\|_{1}\right), \quad z \in W_{2}, h>0, t \in \mathbb{R} .
$$

If $(z(0), \dot{z}(0)) \in H_{3} \times H_{2}, w^{h}(0)=\pi_{h} z(0)$, and $\dot{w}^{h}(0)=\pi_{h} \dot{z}(0)$, then the solutions $z$ and $w$ of (48) and (105) satisfy

$$
\left\|w^{h}(t)-\pi_{h} z(t)\right\|_{1}+\left\|\dot{w}^{h}(t)-\pi_{h} \dot{z}(t)\right\|_{0} \leqslant c_{0} h t\left(\|z(0)\|_{3}+\|\dot{z}(0)\|_{2}\right),
$$

for all $h>0$ and $t \in \mathbb{R}$.

4.5.2. Convergence of the observation systems. The proof of Proposition 4.16 yields:

Proposition 4.21. Assume (95). Consider a family $\left(w^{h}\right)_{h>0}$ in $W^{h}$ and $z \in \mathscr{D}(\mathcal{A})$. If $\mathscr{A}_{h} \mathscr{E}_{h} w^{h} \rightarrow \mathcal{A} z$ in $Z_{0}$ then $\boldsymbol{C}_{h} e^{i t \mathscr{E}_{h}} w^{h} \rightarrow \mathcal{C} e^{i t \mathcal{A}_{z}}$ in $Y$ uniformly on finite intervals of $t$. More precisely, (62) implies the convergence rate

$$
\left\|\left(\boldsymbol{C}_{h} e^{i t \mathscr{E}_{h}} g_{h}^{*} \pi_{h}-\boldsymbol{C}^{i t \mathcal{A}}\right) z\right\| \leqslant\|C\|_{\mathscr{L}\left(H_{2} ; Y\right)} c_{0} h\left(t\|z\|_{3}+2\|z\|_{2}\right),
$$

with $z \in Z_{3}, h>0$, and $t \in \mathbb{R}$.

If $\mathscr{S}_{h} \mathscr{E}_{h} w^{h} \rightarrow \mathcal{A z}$ in $Z_{0}$ then $\mathscr{C}_{h} e^{i t \mathscr{E}_{h}} w^{h} \rightarrow \mathcal{C} e^{i t \mathcal{A}_{z}}$ in $L^{2}(0, T ; Y)$ for all $T>0$.

If $\mathscr{g}_{h} v^{h} \rightarrow z$ in $Z_{0}$ and (48) is admissible then $\varphi_{h} e^{i t \mathscr{E}_{h}} w^{h} \rightarrow C e^{i t A_{z}}$ in $L^{2}(0, T ; Y)$ for all $T>0$.

In the "interior" case, the proof of Proposition 4.10 yields: 
Proposition 4.22. Assume (75). Consider a family $\left(w^{h}\right)_{h>0}$ in $W^{h}$ and $z \in \mathscr{D}(\mathcal{A})$. If $\mathcal{L}_{h} w^{h} \rightarrow z$ in $Z_{0}$ then $\mathcal{C}_{h} e^{i t \mathscr{E}_{h}} w^{h} \rightarrow \mathcal{C} e^{i t \mathcal{A}_{z}} z$ in $Y$ uniformly on finite intervals of $t$. More precisely, (62) implies the convergence rate:

$$
\left\|\left(\mathcal{C}_{h} e^{i t \mathscr{E}_{h}} g_{h}-\boldsymbol{C}^{i t \mathcal{A}}\right) z\right\| \leqslant\|C\| \mathscr{L}\left(H_{1} ; Y\right) c_{0} h\left(t\|z\|_{2}+2\|z\|_{1}\right),
$$

with $z \in Z_{2}, h>0$, and $t \in \mathbb{R}$.

If $\mathcal{L}_{h} w^{h} \rightarrow z$ in $Z_{0}$ then $e_{h} e^{i t E_{h}} w^{h} \rightarrow C e^{i t \mathcal{A}_{z}}$ in $L^{2}(0, T ; Y)$ for all $T>0$.

If $\mathscr{A}_{h} w^{h} \rightarrow z$ in $Z_{-1}$ and (56) is admissible then $\varphi_{h} e^{i t \mathscr{S}_{h}} w^{h} \rightarrow C e^{i t A_{z}}$ in $L^{2}(0, T ; Y)$ for all $T>0$.

4.5.3. Continuous and filtered discrete observability. As in $\S 4.3 .2, \S 4.3 .3$, we consider the semidiscrete system (105) restricted to the filtered space $\mathbf{1}_{\mathscr{E}_{h}^{2}<f(h)} W^{h}$, where $f:\left(0, h_{0}\right) \rightarrow(0,+\infty)$ decreases and $f(h) \rightarrow+\infty$ as $h \rightarrow 0$, and in particular we consider the filtering scale $f(h)=\eta / h^{\sigma}$, where $\eta$ and $\sigma$ are positive parameters.

As in (104), the link between the first and second order filtered spaces is

$$
\mathbf{1}_{\mathscr{g}_{h}^{2}<f(h)} W^{h}=\mathbf{1}_{G_{h}<f(h)} V^{h} \times \mathbf{1}_{G_{h}<f(h)} V^{h} .
$$

Due to Theorem 3.8 and the last part of the Theorem 4.18 with $s=1$ for the direct implications, and due to Theorem 4.17 for the converse implications we obtain:

Theorem 4.23. Assume that the observation operators satisfy (95).

The second order system (48) is admissible (resp. moreover exactly observable) if and only if, for all $\eta>0$ (resp. for $\eta>0$ small enough), there exists $T>0$ such that the semidiscrete system (105) restricted to the filtered space $\mathbf{1}_{g_{h}^{2}<\eta / h^{2 / 3}} W^{h}$, with the $W_{0}^{h}$ norm is admissible (resp. moreover exactly observable) in time $T$ uniformly in $h \in(0,1)$.

In the "interior" case, Theorem 3.13 and the proof of Theorem 4.11 yield:

Theorem 4.24. Assume that the observation operators satisfy (75).

The second order system (56) is admissible (resp. moreover exactly observable) if and only if, for all $\eta>0$ (resp. for $\eta>0$ small enough), there exists $T>0$ such that the semidiscrete system (105) restricted to the filtered space $\mathbf{1}_{\mathscr{g}_{h}^{2}<\eta / h} W^{h}$, with the $W_{-1}^{h}$ norm is admissible (resp. moreover exactly observable) in time $T$ uniformly in $h \in(0,1)$.

Assume $C \in \mathscr{L}\left(H_{0} ; Y\right)$. The second order system (56) is admissible (resp. moreover exactly observable) if and only if, for all $\eta>0$ (resp. for $\eta>0$ small enough), there exists $T>0$ such that the semidiscrete system (74) restricted to the filtered space $\mathbf{1}_{h}^{2}<\eta / h^{4 / 3} W^{h}$, with the $W_{-1}^{h}$ norm is admissible (resp. moreover exactly observable) in time $T$ uniformly in $h \in(0,1)$. 
4.5.4. Convergence of the filtered control systems. The proofs of Theorems 4.19 and 4.14 respectively in the "boundary" case (95) and "interior" case (75) yield:

Theorem 4.25. Assume (95) (resp. assume (75)). Consider a family of initial data $\left(\Psi_{0}^{h}, \Psi_{1}^{h}\right)_{h>0}$ in $W^{h}$ and a family of inputs $\left(u^{h}\right)_{h>0}$ in $L^{2}(0, T ; Y)$ such that $u^{h}$ controls $\left(\Psi_{0}^{h}, \Psi_{1}^{h}\right)$ in time $T$ in the filtered space $\mathbf{1}_{h}^{2}<f(h) W^{h}$, i.e. the solution

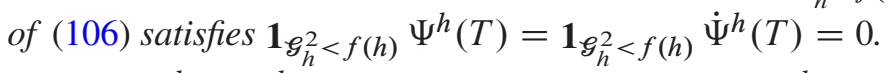

If $\left(J_{h} \Psi_{0}^{h}, J_{h} \Psi_{1}^{h}\right) \rightarrow\left(\zeta_{0}, \zeta_{1}\right)$ weakly in $Z_{-1}$ and $u^{h} \rightarrow u$ weakly in $L^{2}(0, T ; Y)$ then $u$ controls $\left(\zeta_{0}, \zeta_{1}\right)$ in time $T$, i.e. the solution of (49) satisfies $\zeta(T)=\dot{\zeta}(T)=0$.

Assume the discrete system (105) restricted to the filtered space $\mathbf{1}_{g_{h}^{2}<f(h)} W^{h}$ with the $W_{0}^{h}$ norm (resp. the $W_{-1}^{h}$ norm) and the system (48) (resp. the system (57)) are admissible and exactly observable in time T uniformly in $h$. If $\left(J_{h} \Psi_{0}^{h}, J_{h} \Psi_{1}^{h}\right) \rightarrow$ $\left(\zeta_{0}, \zeta_{1}\right)$ in $Z_{-1}$ (resp. in $\left.Z_{0}\right)$ then the minimal control $u^{h}$ of $\left(\Psi_{0}^{h}, \Psi_{1}^{h}\right)$ converges in $L^{2}(0, T ; Y)$ to the minimal control $u$ of $\left(\zeta_{0}, \zeta_{1}\right)$ in (49) (resp. in (57)). E.g. this applies to all $\left(\zeta_{0}, \zeta_{1}\right) \in Z_{-1},\left(\Psi_{0}^{h}, \Psi_{1}^{h}\right)=\left(J^{*} \zeta_{0}, G_{h} J_{h}^{*} \pi_{h} A^{-1} \zeta_{1}\right)$, (resp. to all $\left.\left(\zeta_{0}, \zeta_{1}\right) \in Z_{0},\left(\Psi_{0}^{h}, \Psi_{1}^{h}\right)=\left(J^{*} \pi_{h} \zeta_{0}, J^{*} \zeta_{1}\right)\right)$, cf. Lemma 4.8 .

Remark 4.26. The direct implications in Theorem 4.24 improve Theorem 1.1 in [12] in four ways: the approximation assumption (62) is weaker in practice (cf. Remark 4.1), the assumption on the observation operator $C \in \mathscr{L}\left(H_{\gamma} ; Y\right), \gamma \in[0,1)$, is weakened into $C \in \mathscr{L}\left(H_{1} ; Y\right), A^{-1}$ is not assumed to be compact, the filtering power $\sigma=\min \{2(1-\gamma), 1\}$ in (1.12) in [12] (which is already the author's improvement of Ervedoza's (7.1.12) in [11], cf. [12], Remark 3.1) is improved into $\sigma=1$ for $C \in \mathscr{L}\left(H_{1} ; Y\right)$ and $\sigma=4 / 3$ for $C \in \mathscr{L}\left(H_{0} ; Y\right)$. The converse implications in Theorem 4.24 are not considered in [13].

The second part of Theorem 4.25 in the "interior" case improves Theorem 6.1 in [12] by eliminating the dubiously used assumption (6.11) in [12] which limited to $C \in \mathscr{L}\left(H_{0} ; Y\right)$ the validity of Theorem 6.1 in [12]. The explicit convergence rates of the observations in Proposition 4.22, and the "boundary" case in Theorems 4.25 and 4.23 were not considered in [13].

\section{References}

[1] C. Bardos, G. Lebeau, and J. Rauch, Sharp sufficient conditions for the observation, control, and stabilization of waves from the boundary. SIAM J. Control Optim. 30 (1992), 1024-1065. MR 1178650 Zbl 0786.93009

[2] N. Burq and P. Gérard, Condition nécessaire et suffisante pour la contrôlabilité exacte des ondes. C. R. Acad. Sci., Paris, Sér. I, Math. 25 (1997), 749-752. Zbl 0906.93008

[3] N. Burq and M. Zworski, Geometric control in the presence of a black box. J. Am. Math. Soc. 17 (2004), 443-471. MR 2051618 Zbl 1050.35058 www.ams.org/journals/jams/2004-17-02/S0894-0347-04-00452-7/home.html 
[4] N. Burq and M. Zworski, Bouncing ball modes and quantum chaos. SIAM Rev. 47 (2005), 43-49. MR 2149100 Zbl 1072.81022

[5] G. Chen et al., Exponential decay of energy of evolution equations with locally distributed damping. SIAM J. Appl. Math. 51 (1991), 266-301. MR 1089141 Zbl 0734.35009

[6] N. Cîndea, S. Micu, and M. Tucsnak, An approximation method for exact controls of vibrating systems. SIAM J. Control Optim. 49 (2011), 1283-1305. MR 2818882 Zbl 05959929

[7] S. Dolecki and D. L. Russell, A general theory of observation and control. SIAM J. Control Optimization 15 (1977), 185-220. MR 0451141 Zbl 0353.93012

[8] T. Duyckaerts and L. Miller, Resolvent conditions for the control of parabolic equations. Preprint 2011. hal.archives-ouvertes.fr/hal-00620870

[9] K.-J. Engel and R. Nagel, One-parameter semigroups for linear evolution equations. Springer Verlag, Berlin, 2000. MR 1721989 Zbl 0952.47036

[10] A. Ern and J.-L. Guermond, Theory and practice of finite elements. Springer, New York (NY), 2004. MR 2050138 Zbl 1059.65103

[11] S. Ervedoza, Problèmes de contrôle et stabilisation. Ph.D. thesis. Université de Versailles Saint-Quentin-en-Yvelines, 2008.

[12] S. Ervedoza, Spectral conditions for admissibility and observability of wave systems: applications to finite element schemes. Numer. Math. 113 (2009), 377-415. MR 2534130 Zbl 1170.93013 www.springerlink.com/content/6w57631w2362x732/

[13] S. Ervedoza, Spectral conditions for admissibility and observability for Schrödinger systems: Applications to finite element approximation schemes. Asymptotic Anal. 71 (2011), 1-32. MR 2752768 Zbl 05863558

[14] S. Ervedoza, C. Zheng, and E. Zuazua, On the observability of time-discrete conservative linear systems. J. Funct. Anal. 254 (2008), 3037-3078. MR 2418618 Zbl 1143.65044

[15] L. Gearhart, Spectral theory for contraction semigroups on Hilbert space. Trans. Amer. Math. Soc. 236 (1978), 385-394. MR 0461206 Zbl 0326.47038

[16] S. Hansen and G. Weiss, The operator Carleson measure criterion for admissibility of control operators for diagonal semigroups on $l^{2}$. Syst. Control Lett. 16 (1991), 219-227. MR 1098682 Zbl 0728.93047

[17] I. Herbst, The spectrum of Hilbert space semigroups. J. Oper. Theory 10 (1983), 87-94. MR 715559 Zbl 0535.47024

[18] P. D. Hislop and I. M. Sigal, Introduction to spectral theory. Springer Verlag, New York (NY), 1996. MR 1361167 Zbl 0855.47002

[19] J. S. Howland, On a theorem of Gearhart. Integral Equations Oper. Theory 7 (1984), 138-142. MR 802373 Zbl 0535.47025

www.springerlink.com/content/nq6857322mkj1v71/

[20] Fa Lun Huang, Characteristic conditions for exponential stability of linear dynamical systems in Hilbert spaces. Ann. Differ. Equations 1 (1985), 43-56. MR 834231 Zbl 0593.34048 
[21] J. A. Infante and E. Zuazua, Boundary observability for the space semi-discretizations of the $1-D$ wave equation. M2AN, Math. Model. Numer. Anal. 33 (1999), 407-438. MR 1700042 Zbl 0947.65101 www.esaim-m2an.org/action/displayAbstract?fromPage=online \&aid=8208264

[22] B. Jacob and H. Zwart, On the Hautus test for exponentially stable $C_{0}$-groups. SIAM J. Control Optim. 48 (2009), 1275-1288. MR 2496976 Zbl 1193.47044

[23] T. Kato, Perturbation theory for linear operators. Reprint of the corr. print. of the second ed. 1980. Springer Verlag, Berlin, 1995. MR 1335452 Zbl 0836.47009

[24] G. Lebeau, Contrôle de l'équation de Schrödinger. J. Math. Pures Appl., IX. Sér. 71 (1992), 267-291. MR 1172452 Zbl 0838.35013

[25] J.-L. Lions, Contrôlabilité exacte, perturbations et stabilisation de systèmes distribués. Tome 1: Contrôlabilité exacte. Masson, Paris, 1988. Zbl 0653.93002

[26] K. Liu, Locally distributed control and damping for the conservative systems. SIAM J. Control Optimization 35 (1997), 1574-1590. MR 1466917 Zbl 0891.93016

[27] L. León and E. Zuazua, Boundary controllability of the finite-difference space semidiscretizations of the beam equation. ESAIM, Control Optim. Calc. Var. 8 (2002), 827 862. Volume dedicated to the memory of J. L. Lions. MR 1932975 Zbl 1063.93025 www.esaim-cocv.org/action/displayAbstract?fromPage $=$ online $\&$ aid $=8140812$ Also available at www.numdam.org/numdam-bin/browse? $\mathrm{j}=\mathrm{COCV}$

[28] L. Miller, Réfraction d'ondes semi-classiques par des interfaces franches. C. R. Acad. Sci., Paris, Sér. I, Math. 325 (1997), 371-376. MR 1467089 Zbl 0884.35028

[29] L. Miller, How violent are fast controls for Schrödinger and plates vibrations? Arch. Ration. Mech. Anal. 172 (2004), 429-456. MR 2062431 Zbl 1067.35130 www.springerlink.com/content/d8h4wq6x2wqffuww/

[30] L. Miller, Controllability cost of conservative systems: resolvent condition and transmutation. J. Funct. Anal. 218 (2005), 425-444. MR 2108119 Zbl 1122.93011

[31] L. Miller, A direct Lebeau-Robbiano strategy for the observability of heat-like semigroups. Discrete Contin. Dyn. Syst., Ser. B 14 (2010), 1465-1485. MR 2679651 Zbl 1219.93017 www.aimsciences.org/journals/displayArticles.jsp?paperID=5405

[32] L. Miller, Unique continuation estimates for sums of semiclassical eigenfunctions and null-controllability from cones. Preprint 2008. hal.archives-ouvertes.fr/hal-00411840

[33] K.-D. Phung, Observability and control of Schrödinger equations. SIAM J. Control Optimization 40 (2001), 211-230. MR 1855313 Zbl 0995.93037

[34] J. Prüss, On the spectrum of $C_{0}$-semigroups. Trans. Amer. Math. Soc. 284 (1984), $847-$ 857. MR 743749 Zbl 0572.47030

[35] G. Popov and G. Vodev, Resonances near the real axis for transparent obstacles. Commun. Math. Phys. 207 (1999), 411-438. MR 1724834 Zbl 0951.35036 www.springerlink.com/content/dduwck0rlx8wtmbk/

[36] A. Quarteroni and A. Valli, Numerical approximation of partial differential equations. Springer Verlag, Berlin, 1994. MR 1299729 Zbl 0803.65088

[37] J. V. Ralston, Gaussian beams and the propagation of singularities. Studies in partial differential equations, MAA Stud. Math. 23 (1982), 206-248. MR 716507 Zbl 0533.35062 
[38] K. Ramdani et al., A spectral approach for the exact observability of infinite-dimensional systems with skew-adjoint generator. J. Funct. Anal. 226 (2005), 193-229. MR 2158180 Zbl 1140.93395

[39] K. Ramdani, T. Takahashi, and M. Tucsnak, Uniformly exponentially stable approximations for a class of second order evolution equations - application to LQR problems. ESAIM, Control Optim. Calc. Var. 13 (2007), 503-527. MR 2329173 Zbl 1126.93050

[40] D. L. Russell and G. Weiss, A general necessary condition for exact observability. SIAM J. Control Optimization 32 (1994), 1-23. MR 1255956 Zbl 0795.93023

[41] M. Tucsnak and G. Weiss, Simultaneous exact controllability and some applications. SIAM J. Control Optimization 38 (2000), 1408-1427. MR 1766422 Zbl 0982.93021

[42] M. Tucsnak and G. Weiss, Observation and control for operator semigroups. Birkhäuser, Basel, 2009. MR 2502023 Zbl 1188.93002

[43] G. Weiss, Admissible observation operators for linear semigroups. Isr. J. Math. 65 (1989), 17-43. MR 994732 Zbl 0696.47040 www.springerlink.com/content/215805kq182j8k06/

[44] E. Zuazua, Propagation, observation, and control of waves approximated by finite difference methods. SIAM Rev. 47 (2005), 197-243. MR 2179896 Zbl 1077.65095

[45] H. Zwart, Sufficient conditions for admissibility. Syst. Control Lett. 54 (2005), 973-979. MR 2166288 Zbl 1129.93422

[46] Q. Zhou and M. Yamamoto, Hautus condition on the exact controllability of conservative systems. Int. J. Control 67 (1997), 371-379. MR 1687676 Zbl 0882.93004

Received July 4, 2011; revised November 3, 2011

Luc Miller, MODAL'X, EA 3454, Bât. G, Université Paris Ouest Nanterre La Défense, 200 Av. de la République, 92001 Nanterre Cedex, France.

E-mail: Luc.Miller@math.cnrs.fr 